Simulação numérica 3D do enchimento de compartimentos de reservatórios utilizando o método de elementos finitos

Fernanda Paula Barbosa 


\title{
Simulação numérica 3D do enchimento de compartimentos de reservatórios utilizando o método de elementos finitos
}

\author{
Fernanda Paula Barbosa
}

Orientador: Prof. Dr. Antonio Castelo Filho

Dissertação apresentada ao Instituto de Ciências Matemáticas e de Computação - ICMC-USP, como parte dos requisitos para obtenção do título de Mestre em Ciências - Ciências de Computação e Matemática Computacional.

\section{"VERSÃO REVISADA APÓS A DEFESA"}

Data da Defesa:

$23 / 11 / 2007$

Visto do Orientador:

USP - São Carlos

Dezembro/ 2007 
Dedico este trabalho aos meus pais Cida e João e ao meu noivo Ives, com muito amor. 



\section{Agradecimentos}

Agradeço aos meus pais Cida e João por todo amor, apoio, dedicação e por estarem sempre ao meu lado me apoiando em todos os momentos de minha vida.

Ao meu noivo Ives Renê pelo amor, paciência, por estar ao meu lado compartilhando comigo todos os momentos desde os mais felizes até os mais difíceis. Por nunca me deixar desanimar e pela ajuda constante.

Ao meu irmão Júlio César pelo carinho e incentivo.

Ao Professor Antonio Castelo Filho pela orientação e apoio.

Ao Professor Norberto Mangiavacchi por toda atenção, amizade e pelas contribuições essenciais para o sucesso deste trabalho.

Aos Professores Adenilso da Silva Simão, Luis Gustavo Nonato e Fabrício Simeoni de Sousa pela amizade, pela disponibilidade e apoio intelectual.

Ao Professor Messias Meneguette Junior pela amizade, incentivo e pela orientação durante a graduação.

À toda minha família por torcerem por mim e sempre acreditarem no meu potencial.

À FAPESP e à Furnas Centrais Elétricas pelo suporte financeiro. 

"Suba o primeiro degrau com fé. Você não tem que ver toda a escada. Você só precisa dar o primeiro passo."

(Martin Luther King Jr.) 



\section{Sumário}

Lista de Figuras $\quad$ iv

Lista de Tabelas $\quad$ ix

Resumo xi

Abstract $\quad$ xiii

\begin{tabular}{lll}
\hline 1 & Introdução & 1
\end{tabular}

1.1 Considerações Iniciais . . . . . . . . . . . . . . . . . . . . . . . . . . . . 1

1.2 Metodologia . . . . . . . . . . . . . . . . . . . . . . . 3

1.3 Organização do documento . . . . . . . . . . . . . . . . . . . . . . . . 4

2 Embasamento Teórico $\quad 5$

2.1 Introdução . . . . . . . . . . . . . . . . . . . . . . . 5

2.2 Métodos Numéricos para Solução das Equações . . . . . . . . . . . . . . . 6

2.2 .1 Modelos para Problemas Bidimensionais . . . . . . . . . . . . . 7

2.2 .2 Modelos para Problemas Tridimensionais . . . . . . . . . . . . . . . 9

2.3 Considerações Finais . . . . . . . . . . . . . . . . . . . . . . . . . . 11

\begin{tabular}{|lll}
\hline 3 & Equações Governantes para Escoamento de Fluidos & 13
\end{tabular}

3.1 Introdução . . . . . . . . . . . . . . . . . . . . . . . . . . . . . . . . 13

3.2 Formulações das Equações

3.3 Descrição do Movimento de Fluido . . . . . . . . . . . . . . . . . . . . . . 15

3.4 Equações da Mecânica do Contínuo . . . . . . . . . . . . . . . . . . . . . . 16 
3.4 .1 Equação de Conservação de Massa $\ldots$. . . . . . . . . . . . . . . . . 16

3.4 .2 Equação de Balanço da Quantidade de Movimento . . . . . . . . . 17

3.5 Adimensionalização . . . . . . . . . . . . . . . . . . . . . . . . . . . . . 18

3.6 Condições Iniciais e de Contorno . . . . . . . . . . . . . . . . . . . . . . . 20

3.6.1 Condições para Contornos Rígidos . . . . . . . . . . . . . . . . . . . 20

3.7 Considerações Finais . . . . . . . . . . . . . . . . . . . . . . . . . . . . 21

4 Discretização das Equações Governantes 23

4.1 Introdução . . . . . . . . . . . . . . . . . . . . . . . . 23

4.2 Formulação Variacional . . . . . . . . . . . . . . . . . . . . . . . . . . . . . 24

4.2 .1 Formulação Variacional das Equações de Navier-Stokes . . . . . . . 24

4.3 O Método de Elementos Finitos . . . . . . . . . . . . . . . . . . . . . . . . 27

4.3 .1 Considerações Iniciais . . . . . . . . . . . . . . . . . . . . . . . . . . 27

4.3 .2 Funções de Interpolação . . . . . . . . . . . . . . . . . . . . . . . . 29

4.4 Discretização Espacial . . . . . . . . . . . . . . . . . . . . . . . . . 34

$4.4 .1 \quad$ Método de Galerkin para as Equações de Navier-Stokes . . . . . . . 34

4.4 .2 Cálculo das Matrizes nos Elementos . . . . . . . . . . . . . . . . . . 40

$4.4 .3 \quad$ Integração Numérica . . . . . . . . . . . . . . . . . . . . . . . 46

4.5 Discretização Temporal Pelo Método Semi-Lagrangeano . . . . . . . . . . . 50

4.6 Método de Desacoplamento Baseado em Decomposição LU . . . . . . . . . 51

4.7 Considerações Finais ． . . . . . . . . . . . . . . . . . . . . . . . . . . . . . 53

5 O Simulador GesarSim 3D

5.1 Introdução . . . . . . . . . . . . . . . . . . . . . . . . 55

5.2 Visão Geral do Sistema . . . . . . . . . . . . . . . . . . . . . . . . 56

5.3 Representação do Domínio . . . . . . . . . . . . . . . . . 56

5.4 Método Utilizado no Simulador GesarSim 3D . . . . . . . . . . . . . . . . 58

5.5 Implementação do Método Semi-Lagrangeano . . . . . . . . . . . . . . . . 62

5.6 Considerações Finais $\ldots \ldots \ldots$

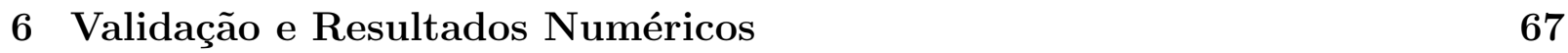

$6.1 \quad$ Introdução . . . . . . . . . . . . . . . . . . . . . . . . . . 67

6.2 Escoamento em um Canal . . . . . . . . . . . . . . . . . . . . 68

6.2.1 Validação Numérica - Perfil de Velocidade Parabólico na Entrada do Canal ......................... 68 
6.2 .2 Conservação de Massa do Sistema . . . . . . . . . . . . . . . . . . 71

6.2.3 Perfil de Velocidade Uniforme na Entrada do Canal - Geometria e Condições de Contorno . . . . . . . . . . . . . . . . . . . 72

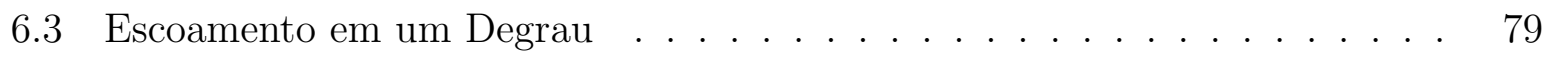

6.3 .1 Geometria e Condições de Contorno . . . . . . . . . . . . . . . . 79

6.4 Escoamento em um Canal com Inclusão de Escadas no Domínio . . . . . . 87

6.4 .1 Geometria e Condições de Contorno . . . . . . . . . . . . . . . 88

6.5 Considerações Finais $\ldots \ldots \ldots \ldots$

\begin{tabular}{llr}
\hline Conclusões & 95
\end{tabular}

7.1 Síntese do Trabalho Desenvolvido . . . . . . . . . . . . . . . . . . 95

7.2 Conclusões . . . . . . . . . . . . . . . . . . . . . . . . . . . . . . . . 96

7.3 Trabalhos Futuros $\ldots \ldots \ldots \ldots$

\begin{tabular}{ll}
\hline Referências Bibliográficas & 103
\end{tabular} 


\section{Lista de Figuras}

4.1 Mapeamento linear do triângulo $\Omega_{e}$ para o elemento mestre $\widehat{\Omega}$ e o mapeamento inverso. . . . . . . . . . . . . . . . . . . . . . . . 31

4.2 Determinação de funções de interpolação para um elemento triangular. . . 32

4.3 Funções de forma para elementos prismáticos lineares. . . . . . . . . . . . . 34

4.4 Elemento mestre cubo $\widehat{\Omega} . \ldots \ldots \ldots$. . . . . . . . . . . . . 42

5.1 Esquema de representação do domínio. . . . . . . . . . . . . . . . 57

5.2 Profundidade $(h)$, elevação $(\eta)$ e superfície de referência . . . . . . . . . . . 60

5.3 Possíveis casos de localização para $p^{*}$ no método semi-lagrangeano. . . . . 63

$6.1 \quad$ Geometria do domínio, o escoamento é da esquerda para a direita. . . . . . 69

6.2 Malha utilizada na simulação vista pelo eixo z. . . . . . . . . . . . . . . . . 69

6.3 Comparação do resultado obtido pelo simulador GesarSim 3D com a solução analítica. . . . . . . . . . . . . . . . . 70

6.4 Malha utilizada nas simulações do caso de escoamento em um canal com vista pelo eixo $z$. . . . . . . . . . . . . . . . . . . . 72

6.5 Domínio para um escoamento em um canal tridimensional. O escoamento é da esquerda para a direita. . . . . . . . . . . . . . . . . . 73

6.6 Condições de contornos utilizadas nas simulações. . . . . . . . . . . . . . . 73

6.7 Simulação de escoamento em um canal com $R e=10$ e cinco camadas de prismas, a) componente de velocidade na direção $x$, b) componente de velocidade na direção $y$, c) componente de velocidade na direção $z$ e d) pressão. 75 
6.8 Simulação de escoamento em um canal com $R e=100$ e cinco camadas de prismas, a) componente de velocidade na direção $x$, b) componente de velocidade na direção $y, \mathrm{c}$ ) componente de velocidade na direção $z$ e d) pressão. 75

6.9 Simulação de escoamento em um canal com $R e=10$ e vinte camadas de \begin{tabular}{|cc}
\hline prismas, a) componente de velocidade na direção $x$, b) componente de ve- \\
\hline
\end{tabular} locidade na direção $y, \mathrm{c}$ ) componente de velocidade na direção $z$ e d) pressão. 76

6.10 Simulação de escoamento em um canal com $R e=1000$ e vinte camadas \begin{tabular}{|c|}
\hline de prismas, a) componente de velocidade na direção $x$, b) componente de \\
\hline
\end{tabular}

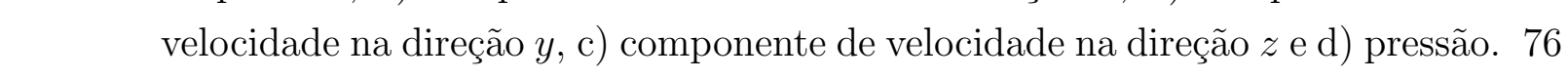

6.11 Simulação numérica do escoamento em um canal com perfil reto: campo

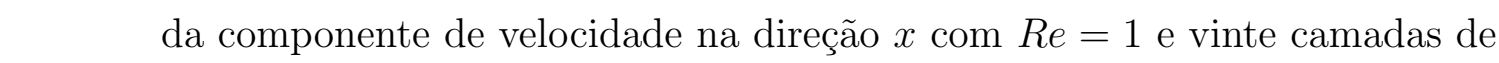
prismas. . . . . . . . . . . . . . . . . . 77

6.12 Simulação numérica do escoamento em um canal com perfil reto: campo da componente de velocidade na direção $x \operatorname{com} R e=100$ e vinte camadas de prismas. . . . . . . . . . . . . . . . . . . 77

6.13 Simulação numérica do escoamento em um canal com perfil reto: campo da componente de velocidade na direção $x$ com $R e=1000$ e vinte camadas de prismas. . . . . . . . . . . . . . . . . . . . 77

6.14 Resultado obtido na superfície do domínio: campo da componente de veloci-

dade na direção $x$ com $R e=10000$ e cinco camadas de prismas, a) resultado
obtido no meio do canal, b) resultado obtido na saída de fluido do canal. . 78

6.15 Resíduo do método de gradiente conjugado nas iterações. . . . . . . . . . . 78

6.16 Malha superficial utilizada nas simulações do caso de escoamento em um

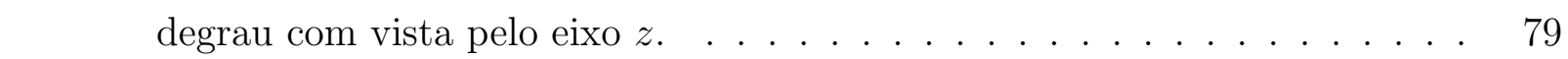

6.17 Geometria e condições de contorno para escoamento em um degrau. A região de entrada de fluido é dada por $0.5 \mathrm{~L} \ldots$. . . . . . . . . . . . . . . 80

6.18 Resíduo do método de gradiente conjugado nas iterações variando o número

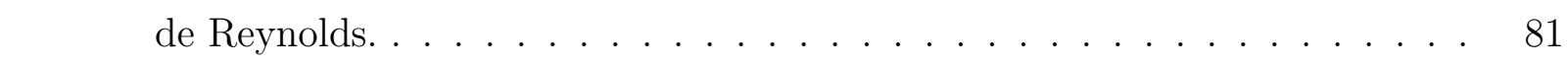

6.19 Campo da componente de velocidade na direção $x \operatorname{com} R e=1$ : a) estado

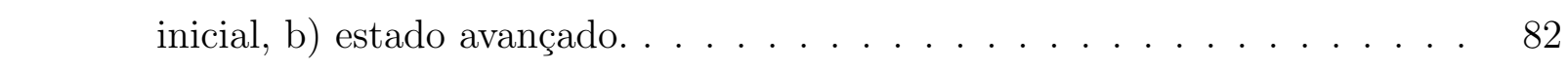

6.20 Campo da componente de velocidade na direção y com $R e=1$ : a) estado

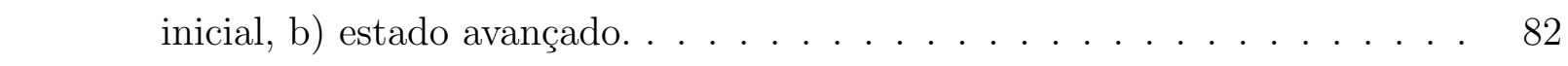

6.21 Pressão resultante da simulação $\operatorname{com} R e=1$ : a) estado inicial, b) estado

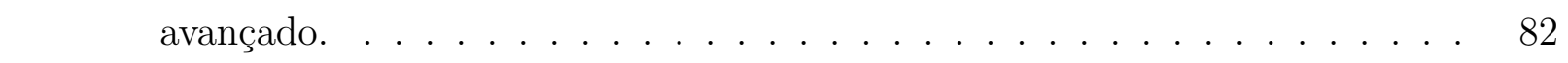


6.22 Campo da componente de velocidade na direção $x \operatorname{com} R e=10$ : a) estado inicial, b) estado avançado. . . . . . . . . . . . . . . . . . 83

6.23 Campo da componente de velocidade na direção y com $R e=10$ : a) estado inicial, b) estado avançado. . . . . . . . . . . . . . . . . . 83

6.24 Pressão resultante da simulação com $R e=10$ : a) estado inicial, b) estado avançado. . . . . . . . . . . . . . . . . . . . . 83

6.25 Campo da componente de velocidade na direção $x \operatorname{com} R e=100$ : a) estado inicial, b) estado avançado. . . . . . . . . . . . . . . . . 84

6.26 Campo da componente de velocidade na direção $y$ com $R e=100$ : a) estado

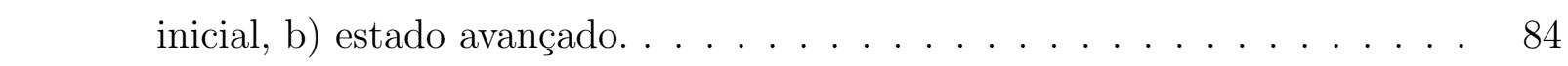

6.27 Pressão resultante da simulação $\operatorname{com} R e=100$ : a) estado inicial, b) estado

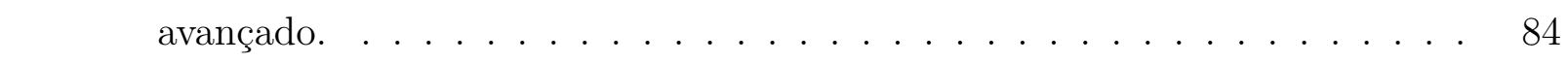

6.28 Campo de velocidade na direção $x \operatorname{com} R e=1000:$ a) estado inicial, b)

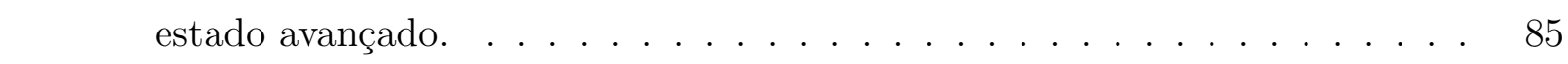

6.29 Campo da componente de velocidade na direção $y$ com $R e=1000$ : a) estado

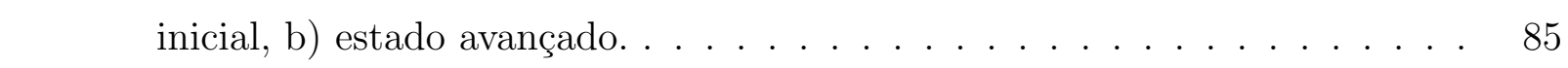

6.30 Pressão resultante da simulação $\operatorname{com} R e=1000$ : a) estado inicial, b) estado

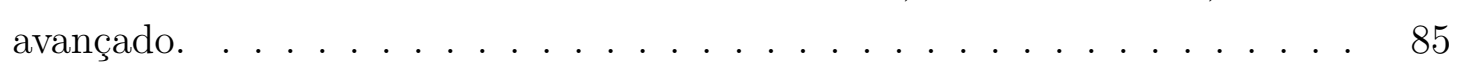

6.31 Simulação numérica do escoamento em um degrau: a) campo da componente de velocidade na direção $x$, b) campo da componente de velocidade \begin{tabular}{|c|}
\hline na direção $y$, b) campo da componente de velocidade na direção $z$ e d) \\
\hline
\end{tabular} pressão, com $R e=1$. . . . . . . . . . . . . . . . . . . . . 86

6.32 Simulação numérica do escoamento em um degrau: a) campo da componente

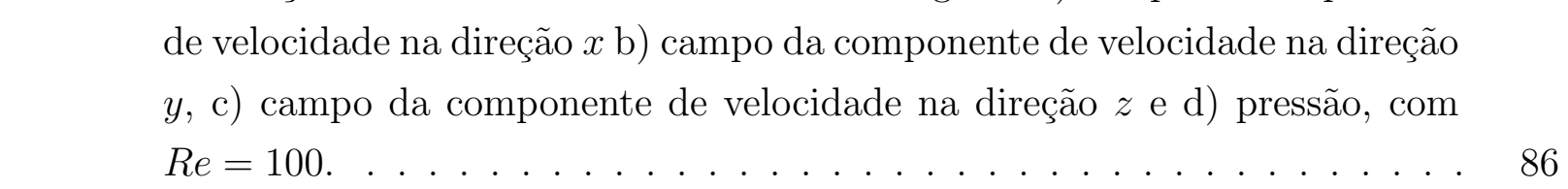

6.33 Simulação numérica do escoamento em um degrau com vista na entrada \begin{tabular}{|c|}
\hline do canal, campo da componente de velocidade na direção $x$ a) $R e=1, \mathrm{~b})$ \\
\hline
\end{tabular}

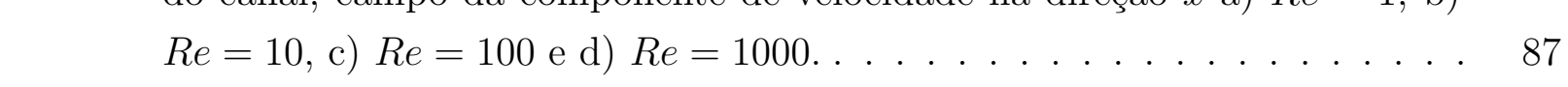

6.34 Perspectiva na direção y e condições de contorno para o caso de inclusão de

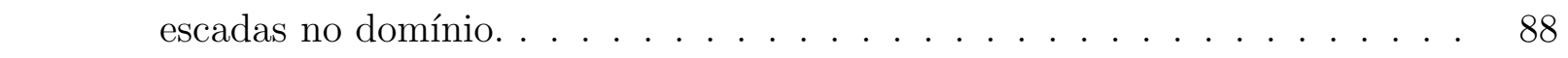

6.35 Detalhe da dimensão de um degrau na escada. . . . . . . . . . . . . . . . 89

6.36 Simulação em um domínio com escadas, campo da componente de velocidade

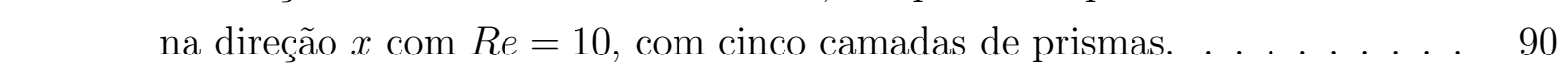


6.37 Simulação em um domínio com escadas, campo da componente de velocidade na direção $x \operatorname{com} R e=100$, com cinco camadas de prismas. . . . . . . . . 90

6.38 Resultado obtido no meio do canal em um domínio com escadas, campo da componente de velocidade na direção $x$ com cinco camadas de prismas, a) $R e=10, \mathrm{~b}) R e=100$. . . . . . . . . . . . . . . . . . . . . 90

6.39 Resíduo do método de gradiente conjugado nas iterações. . . . . . . . . . . 91

6.40 Simulação numérica do escoamento com escadas no domínio: a) campo da componente de velocidade na direção $x, \mathrm{~b}$ ) campo da componente de velo-

\begin{tabular}{|c|}
\hline cidade na direção $y, \mathrm{c})$ campo da componente de velocidade na direção $z$ e \\
\hline
\end{tabular}
d) pressão com $R e=10$. . . . . . . . . . . . . . . . . . . . 91

6.41 Simulação numérica do escoamento com escadas no domínio: a) campo da \begin{tabular}{|ccc}
\hline componente de velocidade na direção $x, \mathrm{~b})$ campo da componente de velo- \\
\hline
\end{tabular} \begin{tabular}{|c|}
\hline cidade na direção $y, \mathrm{c})$ campo da componente de velocidade na direção $z \mathrm{e}$ \\
\hline
\end{tabular}

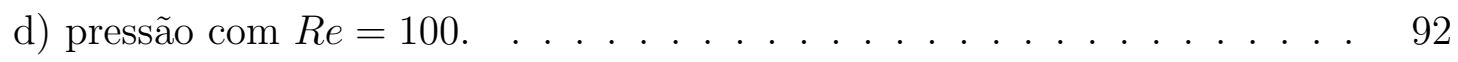

6.42 Simulação numérica do escoamento com escadas no domínio: a) campo da componente de velocidade na direção $x, \mathrm{~b}$ ) vista do campo da componente de velocidade na direção $x$ na região de saída de fluido, c) campo da componente de velocidade na direção y e d) campo da componente de velocidade na direção $z, \operatorname{com} R e=1$. . . . . . . . . . . . . . . . . . . . 93 


\section{Lista de Tabelas}

4.1 Coeficientes abscissa e peso da fórmula de quadratura gaussiana para uma reta. . . . . . . . . . . . . . . . . . . . . . . . . 48

4.2 Coordenadas e pesos da fórmula de quadratura gaussiana para elementos triangulares. . . . . . . . . . . . . . . . . . . . . . . . . 49

4.3 Pontos de quadratura e pesos utilizados na integração para elementos prismáticos lineares. . . . . . . . . . . . . . . . . 50

6.1 Teste da conservação de massa para o escoamento em um canal. . . . . . . 71 
Resumo

A simulação numérica de escoamentos de fluidos em uma grande variedade de aplicações requer a utilização de técnicas numéricas de alta eficiência e recursos computacionais de alto desempenho. O objetivo deste trabalho é iniciar uma investigação de escoamentos de fluido durante o enchimento de compartimentos de reservatórios. Uma abordagem inicial foi tratar problemas de escoamento em um canal, rebuscando a geometria do domínio para contemplar problemas mais complexos. Este trabalho apresenta o desenvolvimento e os resultados obtidos de um método numérico para simulação de escoamento de fluido incompressível em um domínio tridimensional, onde as equações de Navier-Stokes são desenvolvidas em uma formulação euleriana e discretizadas pelo método de elementos finitos. Os termos convectivos destas equações foram tratados pelo método semi-lagrangeano e o método de Galerkin foi utilizado para discretização espacial, um método baseado em decomposição LU foi utilizado para desacoplar as componentes de velocidade e pressão, sendo esta última calculada utilizando-se uma aproximação hidrostática. O domínio tridimensional foi representado por uma malha manipulada por uma estrutura de dados topológica, formada por células que definem elementos prismáticos lineares. Foram realizados experimentos sob várias alterações na geometria do domínio e também sob diferentes condições iniciais. Os resultados mostraram uma boa aproximação do método, quando analisado comparativamente a uma solução analítica. 



\section{Abstract}

The numerical simulation of fluid flow over many applications require the use of numerical techniques of high efficiency and demand high computational power. This work aims at initiating an investigation about fluid flows while filling reservoirs. The initial approach was to deal with fluid flows in a retangular duct, as increasing the complexity of its geometry in order to model more complex cases. This document describes the development of a numerical method for the simulation of incompressible fluid flow over a threedimentional domain, where the Navier-Stokes equations were written under an Eulerian formulation and discretized by the Finite Elements Method. The semi-Lagrangean method was used to discretize the convective terms and the components of velocity and pressure were decoupled through the use of a method based on LU decomposition, where the final pressure was determined by using a hidrostatic aproximation. The threedimentional domain was represented by a mesh, manipulated by a topologic data structure, formed with cells that define linear prismatic elements. Many experiments were performed under different geometries of the domain and also under different initial conditions. The result showed a good approximation of the described method, when compared with an analitical solution. 



\section{Capítulo \\ 1 \\ Introdução}

\subsection{Considerações Iniciais}

O interesse pelo estudo do movimento dos fluidos existe há vários séculos, devido à sua presença em grande parte dos processos que ocorrem na natureza e em muitas aplicações em engenharia. Historicamente, a mecânica dos fluidos preocupou-se em estudar esses movimentos experimentalmente muito antes do que matematicamente Fortuna, 2000, pois a descrição matemática desses movimentos só foi possível a partir do século XIX com as equações de Navier-Stokes.

A solução dos problemas de escoamentos de fluidos requer o tratamento das equações de Navier-Stokes, altamente não-lineares, acopladas às equações da conservação de massa e energia. Em conjunto com condições iniciais e de contorno fisicamente apropriadas, as equações de Navier-Stokes permitem, a princípio, obter informações de caráter fundamental da dinâmica dos fluidos. Desta forma, soluções analíticas das equações de Navier-Stokes só são possíveis para alguns poucos casos bastante simplificados e idealizados.

Com o advento do computador, a partir dos anos de 1950, surgiu a alternativa de se obter soluções numéricas das equações de Navier-Stokes, utilizando técnicas computacionais para a obtenção dos campos de velocidade e pressão, entre outros, que compõem o escoamento. Assim, surgiu uma nova linha de estudos de fluidos, denominada Dinâmica de Fluidos Computacional (CFD - Computational Fluid Dynamics), que é a área da com- 
putação científica que estuda métodos computacionais desenvolvidos para realização de experimentos computacionais ou simulações numéricas de escoamentos em regiões arbitrárias e com condições de contorno gerais, envolvendo escoamentos com ou sem transferência de calor.

A simulação numérica pode ser vista como uma relação entre resultados teóricos e práticos, ou seja, sendo uma nova solução para determinados problemas, despertando o interesse de muitos pesquisadores. Para que a solução numérica seja aplicada a um problema, expressões matemáticas devem ser derivadas. Essas expressões matemáticas geralmente são definidas a partir da aplicação de princípios físicos, descritos por leis e princípios adequados ao fenômeno, como conservação de massa, energia e movimento Anderson, 1995, Fortuna, 2000, Auada, 1997, Panton, 1984.

Ao longo dos anos de seu funcionamento, um reservatório recebe sedimentos suspensos transportados pelos cursos de água. Grande parte desses sedimentos, formados por partículas que podem estar em suspensão ou no leito do rio, percorre longas distâncias e é geralmente depositada na entrada ou ao longo do reservatório, onde a velocidade do escoamento e a capacidade de transporte de material diminuem consideravelmente Sousa, 2006. Uma vez depositadas, estas partículas se consolidam, aumentando sua resistência a erosão e se tornam difíceis de serem carregadas Mohammad, 2003. Uma simulação nesse âmbito se faz necessária uma vez que a formação de reservatórios hidroelétricos tem repercussões severas no ambiente, tanto nos cursos d'água quanto nas suas vizinhanças, interferindo nos ecossistemas e nos modos de vida das populações envolvidas.

Um dos momentos mais críticos é o enchimento do reservatório quando a água se acumula gradualmente. Durante este processo a biomassa terrestre é decomposta, lançando substâncias que se concentram nos volumes dos diferentes compartimentos do reservatório. Este impacto tem sido minimizado com o desmatamento e remoção do material orgânico do leito a ser inundado. No entanto, a limpeza da bacia de acumulação é um procedimento caro, freqüentemente desnecessário para vários compartimentos, e muitas vezes inviável operacionalmente. Ainda é comum a utilização de modelos globais de reservatórios para estabelecer a quantidade de biomassa a ser removida. Estes modelos tratam o reservatório como um corpo único. De fato, como os compartimentos não são a unidade de análise, não se obtêm informações de onde nem do tipo de remoção que deve ser realizado. Muitas vezes a quantidade de biomassa que deve ser removida para se manter índices de qualidade de água aceitáveis é superestimada, ou, não tendo sua localização especificada em cada compartimento, o procedimento é bastante ineficiente. 
Uma ferramenta capaz de simular o comportamento dos futuros compartimentos do reservatório, durante seus respectivos enchimentos, pode fornecer informações mais precisas de onde, quando, qual e quanto material orgânico deve ser retirado. Desta forma, é possível evitar remoções desnecessárias e viabilizar ambiental e economicamente a utilização hidroelétrica de recursos hídricos.

Neste trabalho, a atenção é voltada para o estudo e simulação de escoamentos de fluidos em domínios tridimensionais com aplicação em reservatórios hidroelétricos. No entanto, o enfoque deste trabalho é dar um primeiro passo nessa investigação, propiciando o estudo e desenvolvimento de métodos numéricos para casos mais específicos e complexos, contribuindo desta forma na aplicabilidade das simulações para áreas ambientais. Neste trabalho utilizou-se malhas estruturadas e não estruturadas e o método de elementos finitos [Zienkiewicz and Taylor, 2000], Zienkiewicz and Cheung, 1965], Chung, 1978], na solução numérica das equações governantes que modelam o movimento de fluido.

O uso de malhas não estruturadas para discretização do domínio está cada vez mais presentes nos método numéricos Maliska, 1995, e vem obtendo uma maior atenção dos pesquisadores da área, por se tratar de uma decomposição do domínio muito flexível, e altamente ajustáveis a qualquer tipo de geometria Auada, 1997, Winslow and Harlow, 1967]. Esta facilidade de representação faz com que o uso de malhas não estruturadas seja um caminho natural da evolução dos métodos de aproximação numérica. Na atualidade, duas técnicas de discretização têm sido estudadas e desenvolvidas para este tipo de malha, aproximação por volumes finitos Baliga and Pantankar, 1981, Baliga et al., 1983, Prakash and Pantankar, 1986, Sparrow et al., 1988, Maliska, 1995, Vasconcellos, 1999 e por elementos finitos Oden and Wellford, 1972, Oden et al., 1998.

Este trabalho se insere em um projeto de pesquisa que foi conduzido pelo GESAR Grupo de Ensaios e Simulações Ambientais para Reservatórios, que envolve pesquisadores de outras unidades, cujo objetivo é o desenvolvimento de um software que permita o tratamento de casos reais em compartimentos de reservatórios.

\subsection{Metodologia}

As equações governantes foram desenvolvidas em uma formulação euleriana e discretizadas pelo método de elementos finitos. O método de Galerkin foi utilizado para discretização espacial dos termos difusivos e de pressão. O método semi-lagrangeano foi utilizado na 
discretização dos termos convectivos. O domínio do problema é constituído de elementos prismáticos lineares e para a manipulação da malha foi utilizada uma estrutura de dados topológica. Na superfície do domínio considerou-se um grau de liberdade a mais no centróide dos triângulos nos cálculos de velocidade, aumentando desta forma a eficiência e a estabilidade das soluções numéricas. Foi utilizada uma aproximação hidrostática para o cálculo da pressão no domínio. Um método baseado em uma decomposição LU por blocos possibilitou o desacoplamento das componentes de velocidade e pressão, diminuindo o custo computacional envolvido. Para a solução dos sistemas lineares resultantes, utilizouse o método de gradientes conjugados que soluciona problemas que envolvem matrizes esparsas e simétricas.

\subsection{Organização do documento}

Este documento apresenta a seguinte organização:

- O capítulo 2 os métodos numéricos mais utilizados nas soluções das equações, discute os trabalhos encontrados na literatura relevantes a este, mostrando aplicações dos métodos até então expostos na soluções de problemas na área de mecânica dos fluidos computacional;

- O capítulo 3 apresenta o desenvolvimento das equações de conservação de massa e balanço da quantidade de movimento para escoamento de fluidos escritas em uma formulação euleriana;

- O capítulo 4 apresenta a formulação variacional das equações de Navier-Stokes, uma discussão sobre o método de elementos finitos, descreve o método de Galerkin e o método semi-lagrangeano para discretizar as equações de Navier-Stokes no espaço e no tempo;

- O capítulo 5 apresenta detalhes sobre o desenvolvimento do simulador GesarSim 3D e do método utilizado para resolução das equações;

- O capítulo 6 apresenta a validação do método numérico proposto para a solução das equações de Navier-Stokes e alguns resultados numéricos de simulação;

- O capítulo 7 apresenta as considerações finais e propostas para trabalhos futuros;

- Finalmente são apresentadas as Referências Bibliográficas. 


\section{Capítulo \\ 2 \\ Embasamento Teórico}

Este capítulo apresenta os principais métodos de discretização de modelos matemáticos e os trabalhos relevantes para esta dissertação.

\subsection{Introdução}

Para a obtenção da solução numérica de um problema de escoamento de fluido pode-se seguir a seguinte sequência: dado um problema físico, utiliza-se uma modelagem matemática através de equações governantes e em seguida aplica-se um processo de discretização, obtendo-se uma solução aproximada. Atualmente, o desenvolvimento científico tem se beneficiado enormemente de um novo método científico criado com o objetivo de conectar os métodos prático e teórico: o numérico. O uso de técnicas numéricas para a solução de problemas complexos da engenharia e da física é hoje uma realidade, graças ao desenvolvimento de novos algoritmos, de computadores de alta velocidade e de grande capacidade de armazenamento. Em função dessa disponibilidade computacional, o desenvolvimento de algoritmos para a solução dos mais diversos problemas tem recebido uma enorme atenção dos analistas numéricos. As principais vantagens das soluções numéricas são: baixo custo, evolução temporal do processo, resolução de problemas em geometrias complexas, obtenção de resultados com rapidez dentre outras. 


\subsection{Métodos Numéricos para Solução das Equações}

Um dos principais problemas estudados na área de Dinâmica dos Fluidos Computacional é o desenvolvimento de métodos numéricos para encontrar com maior exatidão possível, a solução de um conjunto de equações. Existem vários métodos com essa finalidade, que se distinguem essencialmente pela forma de aproximar as derivadas presentes nas equações e o uso de malhas distintas, podendo assim ser subdivididos em três classes: métodos de volumes finitos, o método de diferenças finitas e o métodos de elementos finitos.

Os métodos de volumes finitos facilitam a discretização em malhas não-estruturadas, avaliando-se as equações diferenciais integradas em volumes de controle na malha computacional. Um método apresentado em Baliga and Pantankar, 1981 constitui hoje um dos principais métodos para análise numérica de escoamentos e transferência de calor. A característica principal deste método é a fácil interpretação física dos termos das equações em termos de fluxos, fontes e forças, devido ao fato da formulação resultante ser de natureza conservativa uma vez que tal formulação é obtida através dos princípios de conservação. O método de volumes de controle com malhas estruturadas e não estruturadas em coordenadas generalizadas para o tratamento de geometrias irregulares, tem sido implementado por vários grupos de pesquisa e aplicado na solução de problemas de escoamentos e transferência de calor, Silva, 1998.

O método de diferenças finitas tem sido usado para o cálculo de escoamentos de fluidos e transferência de calor; uma limitação deste método está na discretização de domínios com complexidade geométrica, problema este que pode ser parcialmente solucionado pelo uso de malhas não-estruturadas. Normalmente estes métodos são baseados em aproximações por séries de Taylor dos operadores diferenciais presentes nos modelos matemáticos, truncados de acordo com a precisão desejada.

Devido às dificuldades em se utilizar o método das diferenças finitas em geometrias complexas, o método de elementos finitos, inicialmente desenvolvido para análise de estruturas, começou a ser aplicado para o caso de escoamentos, devido à sua grande versatilidade na discretização de domínios geometricamente complexos. O método tornou-se amplamente aceito a partir dos anos 60, quando foram iniciadas pesquisas em várias partes do mundo. Desde então sofreu algumas reformulações e desde 1967, após a inserção do método, podese encontrar uma vasta literatura devotada a teoria e aplicação do método. Algumas referências básicas que tratam da aplicação do método de elementos finitos em escoamentos de fluidos podem ser encontradas em Connor e Brebbia (Connor and Brebbia, 1976), 
Chung ( Chung, 1978), Baker ([Baker, 1983), e Whiting (Whiting, 1999).

Os métodos de elementos finitos podem facilmente ser utilizados sobre uma discretização de malhas não-estruturadas, buscando a solução do problema variacional associado ao problema original, projetando as equações em um espaço cuja base é conhecida e integrando-as no domínio computacional.

A aplicação de um método numérico pode ser dividida em três etapas principais, as quais, na nomenclatura do método de elementos finitos, são: pré-processamento, processamento e pós-processamento. Na etapa de pré-processamento, define-se a geometria ou domínio físico do problema; domínio este discretizado por algum tipo de elemento, constituindo a malha de elementos finitos. Nesta etapa podem ser definidas as propriedades físicas do fluido e demais parâmetros (condições de contorno e iniciais) necessários para a solução do problema. Na fase de processamento, aplica-se um programa baseado no modelo numérico para obtenção das grandezas de interesse (velocidade, pressão, temperatura) em pontos do domínio denominados nós dos elementos. Na etapa de pós-processamento, os resultados são analisados para se verificar a validade do modelo numérico ou os propósitos para os quais se resolveu o problema. Técnicas de visualização gráfica geralmente são empregadas para a análise dos resultados. Uma vez validado o programa, pode-se aplicá-lo para o projeto de modelos de equipamentos onde ocorrem os escoamentos.

\subsubsection{Modelos para Problemas Bidimensionais}

Modelos 2D, que não calculam rigorosamente o perfil hidrodinâmico, entre outros efeitos, na orientação da profundidade, é chamado de modelo de águas rasas, e nele as equações de Navier-Stokes sofrem uma grande simplificação. O trabalho de Cecchi Cecchi et al., 1998 apresenta o esforço de simulação do comportamento da lagoa de Veneza com as equações de Navier-Stokes simplificadas para a aproximação de águas rasas e com uma malha computacional ajustada para o método dos elementos finitos.

As condições de contorno mais frequentes da maioria dos modelos 2D são as entradas e saídas de água, (normalmente as vazões e temperatura são especificadas apenas para as entradas), cisalhamento no fundo, ausência de troca de calor entre o leito de fundo e a coluna d'água, fluxo térmico através da superfície livre e cisalhamento na superfície causado pelo vento.

Embora a hipótese da homogeneização de uma das dimensões resulte numa simplificação, reduzindo assim o esforço numérico de solução do problema quando comparado 
aos modelos tridimensionais, os casos resolvidos por modelos bidimensionais ainda assim são bastante dispendiosos em volume de cálculos e apresentam dificuldades de convergência.

Em hipóteses bidimensionais, onde a dimensão da profundidade é menos representativa que as demais, Basha Basha, 1997 mostrou uma tentativa de acompanhamento do fenômeno de advecção-dispersão em rios com uma distribuição de velocidade axial lateralmente não uniforme. Um modelo bidimensional integrado na profundidade foi proposto em Ye e McCorquodate Ye and McCorquodate, 1997, modelando fluxos turbulentos num sistema de coordenadas curvilíneas ajustadas ao contorno, baseando-se no método de volumes-finitos.

Em Sousa Sousa, 2005 é proposto um método numérico para a solução das equações de conservação que modelam o escoamento de vários fluidos imiscíveis, sobre uma malha de elementos finitos que se move com velocidade arbitrária no domínio. A interface é discretizada pelos próprios elementos computacionais da malha, e a aplicação da tensão interfacial é feita através da distribuição da força nos nós livres vizinhos à interface. Para a solução das equações de Navier-Stokes foi utilizado um método de projeção baseado em decomposição LU por blocos.

Soluções temporais foram obtidas em [Stockstill et al., 1997] a partir de condições iniciais especificadas que usaram uma representação de elementos finitos móveis, do tipo Petrov-Galerkin implícito, para as equações fundamentais. O modelo de elementos finitos móveis produziu uma solução simultânea para o deslocamento dos contornos e as variáveis de fluxo, gerando soluções estáveis até mesmo para escoamentos supercríticos com números de Courant relativamente grandes. Outro modelo de elementos finitos bidimensional que utilizou um método de Petrov-Galerkin foi desenvolvido em Berger and Stockstill, 1995 e resultou num sistema robusto para modelar canais de alta velocidade.

Em Wille, 1998, foram desenvolvidos algoritmos para operadores de desacoplamento nodal em elementos finitos adaptáveis para as equações de Navier-Stokes. Os algoritmos de solução nodal consistem em dividir as equações de Navier-Stokes em sistemas de equações que são resolvidos seqüencialmente. Um bom exemplo da utilização das técnicas de discretização e ajustes pode ser encontrado em Cecchi et al., 1998, onde a dinâmica de águas rasas foi estudada e um algoritmo para esta dinâmica foi desenvolvido usando-se um método semi-implícito.

Um método semi-lagrangeano é apresentado em Karpik and Crockett, 1997 para a solução da equação de advecção-difusão em geometrias complexas. Foi também proposto um modelo semi-implícito em elementos finitos. Para a discretização do espaço no domínio 
horizontal, foram empregados elementos lagrangenos quadráticos e isoparamétricos de nove nós. Uma fórmula de interpolação lagrangeana cúbica foi utilizada com a finalidade de estimar os gradientes verticais dos dois componentes de velocidades horizontais.

\subsubsection{Modelos para Problemas Tridimensionais}

Os maiores empecilhos ao uso e desenvolvimento dos modelos 3D são a grande exigência computacional e o enorme conjunto de parâmetros a serem ajustados, necessários à montagem dos problemas. A aplicação de modelos 3D ainda é uma tarefa que envolve equipes altamente especializadas, mediações intensivas de dados de campo e supercomputação. Portanto estes recursos estão ainda longe do alcance das atividades de avaliação preliminares, devido aos custos elevados.

Um estudo abrangente sobre modelagem e simulação de sistemas aquáticos em ambientes de geoprocessamento é apresentado por Soares Soares, 2003. O trabalho apresenta metodologias computacionais de representação de compartimentos de reservatórios. Apresenta também técnicas para geração de malhas bidimensionais em elementos triangulares e tridimensionais em elementos tetraédricos, além de um conjunto de ferramentas auxiliares para mapeamento espacial, apresentadas através de aplicações em exemplos hipotéticos e estudos de casos reais. O trabalho também apresenta uma metodologia em elementos finitos, incluindo esquemas de Galerkin e Petrov-Galerkin.

A respeito de problemas de reservatórios, um modelo numérico tridimensional de hidrodinâmica e de transporte de massa chamado LMT3D, usado nas simulações da qualidade de água em mares e em lagos litorais, é apresentado em Rajar e Cetina Rajar and Cetina, 1997b. Este modelo utiliza um esquema numérico híbrido de volumes finitos e de diferenças finitas com o intuito de diminuir os efeitos de difusão numérica. Três casos deste campo foram estudados, como a modelagem $3 D$ da circulação e do transporte de nutrientes em um lago alpino; uma modelagem $2 D$ da ciclagem de mercúrio na baía de Trieste e uma simulação $3 D$ de longo termo da dispersão de contaminantes radioativos no mar do Japão. Tais casos podem ser encontrados mais detalhadamente em Rajar e Cetina Rajar and Cetina, 1997a.

O trabalho de $\mathrm{Wu}$ Wu et al., 2000] apresentou um modelo numérico em três dimensões para cálculo de escoamento e transporte de sedimentos em um canal de laboratório em foma de U, com modelo de turbulência $k-\epsilon$ Patankar and Spalding, 1972 e discretização das equações por método de volumes finitos. O trabalho defendeu a necessidade da simulação 
em 3D, para a captação dos escoamentos secundários na reprodução de escoamentos reais, negligenciada pelas simulações 1D e 2D, na maioria nessa linha de pesquisa.

Fluxos em trechos de rios naturais foram modelados no espaço tridimensional discretizado em diferenças finitas, Sinha and Odgaard, 1998. Este modelo resolveu as equações RANS (Reynolds-Averaged-Navier-Stokes) associadas a um modelo de turbulência padrão, e as múltiplas ilhas do leito foram resolvidas diretamente empregando-se coordenadas curvilíneas ajustadas ao contorno juntamente com uma aproximação de multiblocos.

Um modelo matemático tridimensional de fluxos de superfície livre foi aplicado em Ye e Mc Corquodale $Y$ Ye and McCorquodale, 1998 para simular os fluxos e transporte de massa em um canal encurvado. No plano horizontal, foi usado um sistema de coordenadas curvilíneas ajustado ao canal, considerando que, no plano vertical, a transformação foi adotada para mapear a superfície livre e a topografia variável do leito. O trabalho empregou o método dos elementos finitos.

Um método numérico eficiente para cálculos hidrodinâmicos em três dimensões foi proposto por Lu e Wai Lu and Wai, 1998, baseado no operador de desacoplamento em conjunto com um método euleriano-lagrangeano, o método de elementos finitos combinado com o método de diferenças finitas. O método do operador de desacoplamento foi empregado para dividir as equações de quantidade de movimento em três partes, aumentando a eficiência e a estabilidade das soluções numéricas.

Um trabalho que apresenta a implementação em paralelo do método dos elementos finitos aplicado na resolução de sistemas não-lineares não-simétricos obtidos a partir da discretização das equações que governam o comportamento hidrodinâmico do escoamento em águas rasas foi feito por Slobodcicov [Slobodcicov, 2003.

Nas últimas décadas dentre as simulações 3D, podemos citar Wang e Adeff Wang and Adeff, 1986 baseado em elementos finitos, e Lin e Falconer Lin and Falconer, 1996 com um modelo para regiões costeiras e estuários, nos quais adotou-se um modelo simples de viscosidade turbulenta (constante). Van Rijn [Rijn, 1987] estabeleceu um modelo no qual o transporte de sedimentos é calculado com uma aproximação 3D e a hipótese de escoamento com integração na vertical, em combinação com um perfil logarítmico de velocidade. Demuren e Rodi [Demuren and Rodi, 1987 usaram um modelo $k-\epsilon$ em simulações 3D de canais meandrados. Demuren Demuren, 1989 estendeu este trabalho, incluindo transporte de sedimentos em suspensão. Em 1991, Demuren inclui também um modelo de transportes de sedimentos junto ao leito (transporte de fundo) e simulou o trabalho experimental de Odgaard e Bergs Odgaard and Bergs, 1988 
, de um canal em forma de U. Demuren Demuren, 1991 usou o modelo 3D de escoamento e transporte de sedimentos FAST3D, desenvolvido na Universidade de Karlsruhe, com adaptação para escoamentos livres.

\subsection{Considerações Finais}

Neste capítulo foi apresentada uma breve discussão sobre as principais técnicas numéricas desenvolvidas para aproximar fenômenos físicos. Estas técnicas, na sua grande maioria, buscam a representação discreta destes processos, tanto na representação geométrica do problema, quanto na discretização dos modelos teóricos existentes. Por fim, foi realizada uma discussão sobre trabalhos encontrados na literatura relevantes para este trabalho, mostrando aplicações dos métodos até então expostos na soluções de problemas na área de mecânica dos fluidos computacional.

Após obter um domínio específico e representado por uma estrutura de dados, o próximo passo é obter as equações relacionadas ao problema em questão. No próximo capítulo, serão apresentadas as equações governantes para escoamento de fluidos. 


\section{Capítulo \\ 3 \\ Equações Governantes para Escoamento de Fluidos}

Neste capítulo, as equações que governam o escoamento de um fluido são apresentadas. Estas equações são obtidas usando princípios de conservação de massa para obtenção da equação da continuidade e aplicação da segunda lei de Newton para obtenção da equação de balanço da quantidade de movimento e estas são aplicadas ao escoamento de fluidos. Após a derivação das equações governantes, são descritas as condições de contorno utilizadas nas simulações deste trabalho.

\subsection{Introdução}

A dinâmica de fuidos é a ciência que descreve o movimento de fuidos e estuda os fenômenos que envolvem fuidos em movimento. No estudo dos escoamentos de fuidos incompressíveis, a modelagem matemática das leis de conservação é bem estabelecida pelas equações de conservação de massa e de Navier-Stokes.

O objetivo da dinâmica dos fluidos é determinar as propriedades de um fluido; para isto, deve-se conhecer as variáveis que determinam o estado dos fluidos, as quais dependem em geral da posição no espaço e do tempo. Conseqüentemente, para conhecer o estado de um fluido, deve-se determinar o valor das variáveis que o identificam, ao longo do tempo 
em cada ponto do espaço ocupado pelo fluido. As variáveis que identificam o estado de um fluido incompressível e isotérmico são: a velocidade u em cada ponto (que é uma grandeza vetorial) e a pressão $p$ (que em cada ponto é uma grandeza escalar).

No entanto, essas variáveis são agrupadas em equações, que passaram a ser conhecidas como equações de Navier-Stokes. Mais detalhes sobre as equações de Navier-Stokes e as propriedades de escoamento de fluidos podem ser encontrados em Batchelor, 1970, Peyret and Taylor, 1983, Panton, 1984, Flecther, 1992, Maliska, 1995, Anderson, 1995 e Ferziger and Períc, 1999. Neste capítulo são apresentadas as equações de interesse escritas em uma formulação euleriana e as condições de contorno utilizadas neste trabalho.

\subsection{Formulações das Equações}

O movimento de um fluido em uma dada região do espaço pode ser especificado de duas maneiras: por uma formulação lagrangeana ou euleriana.

Na formulação lagrangeana, define-se uma região material formada por um conjunto de partículas de fluido, denominada de volume de controle lagrangeano. Conforme as partículas se movimentam no escoamento, a região se deforma, e não há fluxo de massa através de suas faces. Nesta formulação, as grandezas do escoamento são especificadas como função do tempo e da partícula de fluido.

Na formulação euleriana, define-se uma região fixa no espaço, que não se deforma em relação ao tempo. Neste caso, há fluxo de massa pelas faces do volume de controle, e as equações para o escoamento são determinadas a partir do balanço de fluxo deste volume de controle.

Existe também uma forma geral de se descrever o movimento de um fluido, chamada de formulação lagrangeana-euleriana arbitrária. Nesta formulação, as equações são desenvolvidas em um referencial se movendo com velocidade arbitrária. Quando este referencial se move com a mesma velocidade do fluido, obtém-se as equações na formulação lagrangeana, e quando o referencial está parado, obtém-se a formulação euleriana.

Neste capítulo, as equações de conservação serão desenvolvidas utilizando-se uma formulação euleriana, onde as propriedades macroscópicas do escoamento dependem das coordenadas espaciais e temporais, como por exemplo, velocidade $\mathbf{u}=\mathbf{u}(\mathbf{x}, t)$, massa específica $\rho=\rho(\mathbf{x}, t)$ e viscosidade $\mu=\mu(\mathbf{x}, t)$. 


\subsection{Descrição do Movimento de Fluido}

Segundo Aris Aris, 1962, o movimento de um fluido pode ser descrito por meio de uma transformação, supondo que em um dado instante de tempo uma determinada partícula esteja em uma posição $\xi \in \mathbb{R}^{m}$ e num tempo posterior, a mesma partícula esteja na posição $\mathbf{x} \in \mathbb{R}^{m}$. Admitindo que $\mathbf{x}$ é uma função de $t$ e da posição inicial $\xi$, então

$$
\mathbf{x}=\mathbf{x}(\xi, t)
$$

para todo tempo $t$. As coordenadas iniciais $\xi$ são coordenadas materiais (ou lagrangeana) e as coordenadas x são coordenadas espaciais (ou euleriana). Assumindo que o movimento do fluido é uma função contínua, pode-se inverter (3.1) para recuperar as coordenadas materiais da partícula

$$
\xi=\xi(\mathbf{x}, t)
$$

A descrição lagrangeana da variação de uma propriedade $\phi(\xi, \mathbf{t})$ pode ser vista na descrição euleriana $\phi(\mathbf{x}, t)$ da seguinte maneira [Ferreira, 2001,

$$
\phi(\mathbf{x}, t)=\phi(\xi(\mathbf{x}, \mathbf{t}), \mathbf{t})
$$

Existem duas propriedades a considerar, associadas às descrições lagrangeana e euleriana: a derivada em relação ao tempo mantendo-se $x$ constante e a derivada em relação ao tempo mantendo-se $\xi$ constante. A segunda derivada é frequentemente utilizada em dinâmica de fluidos e é conhecida como derivada tota $\bigsqcup^{1}$ da propriedade de $\phi$.

Derivando $\phi(\mathbf{x}, t)$ em relação ao tempo, mantendo $\xi$ constante, tem-se:

$$
\begin{aligned}
& \frac{\partial \phi}{\partial t}(\mathbf{x}, t)=\frac{\partial \phi}{\partial t}\left(x_{1}(\xi, t), x_{2}(\xi, t), \ldots, x_{m}(\xi, t)\right) \\
& =\frac{\partial \phi}{\partial x_{1}} \frac{\partial x_{1}}{\partial t}+\frac{\partial \phi}{\partial x_{2}} \frac{\partial x_{2}}{\partial t}+\ldots+\frac{\partial \phi}{\partial x_{m}} \frac{\partial x_{m}}{\partial t}+\frac{\partial \phi}{\partial t}
\end{aligned}
$$

Sendo

$$
\frac{\partial x_{i}}{\partial t}(\xi, t)=u_{i}, i=1,2, \ldots, m
$$

obtém-se a derivada total de $\phi(\mathbf{x}, t)$, denotada por $\frac{D \phi}{D t}$,

\footnotetext{
${ }^{1}$ Também conhecida como derivada material ou substancial.
} 


$$
\frac{D \phi}{D t} \equiv u_{1} \frac{\partial \phi}{\partial x_{1}}+u_{2} \frac{\partial \phi}{\partial x_{2}}+\ldots+u_{m} \frac{\partial \phi}{\partial x_{m}}+\frac{\partial \phi}{\partial t},
$$

$\mathrm{Na}$ notação vetorial

$$
\frac{D \phi}{D t}=\frac{\partial \phi}{\partial t}+(\mathbf{u} \cdot \nabla) \phi
$$

que corresponde à derivada total ou material de $\phi$ utilizando-se uma formulação euleriana.

A discussão completa da cinemática do movimento de um fluido pode ser encontrada em Aris, 1962.

\subsection{Equações da Mecânica do Contínuo}

Como pode ser visto em Panton, 1984, as equações da mecânica do contínuo podem ser derivadas do teorema do transporte de Reynolds Aris, 1962. Essas equações que modelam o escoamento de fluidos são obtidas pela aplicação dos seguintes princípios:

- Conservação de massa;

- Balanço da quantidade de movimento (segunda lei de Newton);

- Conservação de energia (primeira lei da termodinâmica).

O fluido será considerado como um contínuo de matéria, isso quer dizer que a estrutura molecular do material é ignorada, e assume-se que é possível definir variáveis físicas como pressão, velocidade e densidade num ponto do fluido. Além disso, os valores dessas variáveis mudam suavemente e então a diferenciação é permitida.

No presente trabalho, as equações de interesse são as equações de conservação de massa e de balanço da quantidade de movimento apresentadas a seguir.

\subsubsection{Equação de Conservação de Massa}

O princípio de conservação de massa estabelece que na ausência de fontes de massa ou sumidouros de massa, toda a massa que entra em um sistema deve sair e/ou se acumular no sistema. O princípio de conservação de massa é expresso pela equação da continuidade:

$$
\frac{D \rho}{D t}+(\rho \nabla) \cdot \mathbf{u}=0
$$


onde $\rho$ é a massa específica, $\nabla$ é o operador gradiente, $\mathbf{u}=(u, v, w)$ é o vetor velocidade e $\frac{D}{D t}$ é a derivada total, que para o caso tridimensional é definida como:

$$
\frac{D \rho}{D t}=\frac{\partial \rho}{\partial t}+u \frac{\partial \rho}{\partial x}+v \frac{\partial \rho}{\partial y}+w \frac{\partial \rho}{\partial z} ;
$$

Como a massa específica de uma partícula material não se altera no tempo, para o caso de escoamentos incompressíveis, tem-se:

$$
\frac{D \rho}{D t}=0
$$

e substituindo em (3.8) tem-se

$$
\rho \nabla \cdot \mathbf{u}=0
$$

tendo que $\rho \neq 0$, então

$$
\nabla \cdot \mathbf{u}=0
$$

A equação (3.11) é conhecida como equação de continuidade utilizada para escoamentos incompressíveis.

\subsubsection{Equação de Balanço da Quantidade de Movimento}

Para obtenção da equação de balanço da quantidade de movimento linear aplica-se a segunda lei de Newton a uma partícula de fluido. Na forma vetorial, a equação de balanço da quantidade de movimento é dada por

$$
\frac{D(\rho \mathbf{u})}{D t}=\nabla \cdot \sigma+\rho \mathbf{g}
$$

onde $\sigma$ representa o tensor de tensões totais de escoamento e $\mathbf{g}$ o campo gravitacional. Utilizando um modelo newtoniano, este tensor pode ser escrito como:

$$
\sigma=-p \mathbf{I}+\tau
$$

onde $p=p(\mathbf{x}, t)$ representa o campo de pressão do escoamento, I é o tensor identidade e $\tau$ é o tensor de tensões viscosas que devido a condição de incompressibilidade é dado por:

$$
\tau=2 \mu \mathbf{D} .
$$

O tensor $\mathbf{D}$ é denominado tensor deformação, e segundo o modelo newtoniano, é dado 
por:

$$
\mathbf{D}=\frac{1}{2} \dot{\gamma}(\mathbf{u})=\frac{1}{2}\left(\nabla \mathbf{u}+\nabla \mathbf{u}^{T}\right)
$$

onde $\dot{\gamma}(\mathbf{u})$ é denominado de tensor taxa de deformação.

Desta forma, utilizando o tensor de tensões newtoniano, pode-se calcular o divergente do tensor de tensões totais:

$$
\nabla \cdot \sigma=\nabla \cdot\left[-p \mathbf{I}+\mu\left(\nabla \mathbf{u}+\nabla \mathbf{u}^{T}\right)\right]=-\nabla p+\nabla \cdot\left[\mu\left(\nabla \mathbf{u}+\nabla \mathbf{u}^{T}\right)\right]
$$

Assim, a equação de balanço da quantidade de movimento pode ser expressa como:

$$
\frac{D(\rho \mathbf{u})}{D t}=-\nabla p+\nabla \cdot\left[\mu\left(\nabla \mathbf{u}+\nabla \mathbf{u}^{T}\right)\right]+\rho \mathbf{g}
$$

As equações da continuidade (3.11) e de balanço da quantidade de movimento linear 3.17) são chamadas de equações de Navier-Stokes que modelam escoamentos incompressíveis.

\subsection{Adimensionalização}

A maior dificuldade de implementação das equações 3.11) e 3.17) está em fixar propriedades físicas que satisfaçam os mais diversos fluidos. Com isto a opção por variáveis adimensinais torna-se muito relevante, onde as grandezas dimensionais podem ser agrupadas em parâmetros adimensionais, que passam a caracterizar o escoamento, surgindo então alguns grupos adimensionais como o número de Reynolds e Froude por exemplo. Porém, é importante frisar que os parâmetros tomados como referência para a adimensionalização devem ser definidos de acordo com as características geométricas, cinemáticas e dinâmicas do problema a ser considerado.

Alguns parâmetros importantes que surgem no processo de adimensionalização relacionados com as equações de Navier-Stokes e utilizados neste trabalho são:

- Número de Reynolds (Re): Representa a razão entre as forças inerciais, que são responsáveis pelo movimento do fluido, e as forças viscosas, que são responsáveis pela 
dissipação devido ao coeficiente de viscosidade molecular do escoamento

$$
R e=\frac{\rho_{0} L U}{\mu_{0}}=\frac{L U}{\nu_{0}},
$$

onde $\rho_{0}$ é a densidade, $L$ é a escala de comprimento, $U$ é a escala de velocidade, $\mu_{0}$ é o coeficiente de viscosidade e $\nu_{0}$ é o coeficiente de viscosidade cinemática e é a razão entre a viscosidade aparente ou dinâmica $\mu_{0}$ e a massa específica $\rho_{0}$.

Em um escoamento, se $R e<1$, tem-se que as forças viscosas são predominantes e será possível desprezar os efeitos de inércia, enquanto que $R e>1$ significa uma predominância das forças inerciais, e neste caso pode-se desprezar os efeitos da viscosidade e considerar o problema como "invíscido". Quando Re $\gg 1$ as forças viscosas são importantes apenas em regiões adjacentes às superfícies sólidas, denominadas de camada limite. O número de Reynolds indica também se o escoamento possui característica laminar $\left(R e<R e_{\text {critico }}\right)$ ou turbulenta (caso contrário).

- Número de Froude $(F r)$ : Representa a razão entre as forças inerciais e as forças gravitacionais, isto é

$$
F r=\frac{U}{\sqrt{g L}}
$$

O número de Froude caracteriza escoamentos nos quais gravidade tem papel importante como é o caso de escoamentos com superfícies livres. Se $F r<1$, há predominância das forças gravitacionais sobre as inerciais no escoamento.

Para adimensionalizar as equações de continuidade e balanço da quantidade de movimento, definem-se algumas variáveis adimensionais, como segue:

$$
\rho=\rho_{0} \rho^{*} \quad \mu=\mu_{0} \mu^{*} \quad p=\rho_{0} U^{2} p^{*} \quad \mathbf{x}=L \mathbf{x}^{*} \quad \mathbf{u}=U \mathbf{u}^{*} \quad t=\frac{L}{U} t^{*} \quad \mathbf{g}=g_{0} \mathbf{g}^{*}
$$

onde $\mathbf{x}=(x, y), L$ é um comprimento característico do escoamento, $U$ uma velocidade característica, $\rho_{0}$ é a massa específica e $g_{0}$ a gravidade.

Para os operadores diferenciais:

$$
\frac{\partial}{\partial t}=\frac{U}{L} \frac{\partial}{\partial t^{*}} \quad \text { e } \quad \nabla=\frac{1}{L} \nabla^{*}
$$

Substituindo-se as variáveis adimensionalizadas e os parâmetros adimensionais $\mathrm{Re}$ e $\mathrm{Fr}$ e eliminando-se (*) para simplificar a notação, obtêm-se 


$$
\begin{gathered}
\nabla \cdot \mathbf{u}=0 \\
\frac{D(\rho \mathbf{u})}{D t}=-\nabla p+\frac{1}{R e} \nabla \cdot\left[\mu\left(\nabla \mathbf{u}+\nabla \mathbf{u}^{T}\right)\right]+\frac{1}{F r^{2}} \rho \mathbf{g}
\end{gathered}
$$

\subsection{Condições Iniciais e de Contorno}

É importante para a formulação dos problemas modelados por equações diferenciais parciais escolher as condições iniciais e de contorno que sejam apropriadas. A condição apropriada para as equações 3.11 e 3.17 é a especificação no domínio de um campo inicial de velocidade, satisfazendo a equação de continuidade $\nabla \cdot \mathbf{u}=0$.

\subsubsection{Condições para Contornos Rígidos}

Neste trabalho, as condições utilizadas para contornos rígidos aplicadas na simulação dos escoamentos foram:

- Condição sem escorregamento ("no-slip"): Esta condição impõe que o fluido deve ser "colado" à superfície, a qual é conhecida como não-escorregadia ("no-slip"). Quando tem-se uma superfície sólida e sem deslizamento de fluido em um escoamento viscoso, é apropriado definir a velocidade tangencial $u_{t}$ do fluido em relação à parede como sendo igual a zero. Desta forma, o fluido que está adjacente à superfície da parede estará em repouso em relação à parede. A componente normal $u_{n}$ da velocidade do fluido também é nula, pois o fluido não pode penetrar na parede.

- Condição de entrada de fluido ("inflow"): Esta condição é usada em fronteiras onde há entrada de fluido no sistema. As condições de contorno para entrada de fluido são dadas por

$$
u_{n}=u_{\text {inflow }} \quad \text { e } \quad u_{t}=0
$$

onde $u_{n}$ é a componente normal ao contorno e $u_{t}$ é a componente tangencial ao contorno.

- Condição de saída de fluido ("outflow"): Esta condição é usada em fronteiras onde há saída de fluido no sistema. Existem várias formas de prescrever condições 
de contorno para saída de fluido, uma condição muito utilizada para esta fronteira é especificar um escoamento paralelo, dada por:

$$
\mathbf{u}_{t} \quad \text { e } \quad \sigma^{n n}=0
$$

onde $\sigma^{n n}$ representa a tensão normal à fronteira de saída de fluido.

Logo, em uma fronteira de saída, onde as componentes tangenciais da velocidade são nulas, pela equação da continuidade 3.20 tem-se que $\frac{\partial \mathbf{u}_{n}}{\partial n}=0$, e da equação 3.23 obtém-se $p=0$ na fronteira de saída de fluido para problemas adimensionalizados.

\subsection{Considerações Finais}

Neste capítulo foram apresentados algumas definições importantes utilizados na formulação das equações governantes do escoamento de fluidos e também o conceito de derivada material.

As equações de conservação, compreendidas pela equação da conservação de massa (chamada também de equação da continuidade) e pela equação de balanço da quantidade de movimento, foram deduzidas a partir de princípios físicos. Por fim, as equações foram escritas em sua forma adimensional e as condições de contorno para o domínio em questão foram definidas.

Obtidas as equações relacionadas ao problema em questão, o próximo passo é escolher qual o tipo de discretização deve ser utilizada para se obter bons resultados. No próximo capítulo as equações governantes são escritas em sua formulação variacional e o método de elementos finitos utilizado na discretização das equações é apresentado juntamente com o método de Galerkin utilizado na discretização espacial de tais equações. 
Capítulo

4

\section{Discretização das Equações Governantes}

Este capítulo descreve a formulação variacional e discretização das equações de Navier-

Stokes pelo método de elementos finitos. É feita uma apresentação do método de Galerkin e do método semi-lagrangeano para discretização espacial e temporal das equações, respectivamente. Em seguida, um método para o desacoplamento das propriedades é descrito para a resolução do sistema de equações diferenciais resultantes da discretização das equações.

\subsection{Introdução}

A principal dificuldade para se resolver as equações de Navier-Stokes é provocada pela não linearidade dos termos que representam as acelerações convectivas. Não existe um procedimento analítico geral para resolver equações diferenciais parciais não lineares e cada problema deve ser considerado individualmente.

A aplicação de elementos finitos em escoamentos incompressíveis envolve duas fontes potenciais de instabilidades associadas com a formulação clássica do problema de Galerkin. Uma das fontes deve-se à presença de termos de convecção nas equações governantes, o que pode resultar em oscilações nos resultados, principalmente no campo de velocidade. Tais oscilações tornam-se mais pronunciadas em escoamentos dominados por altos números de Reynolds. A outra fonte de instabilidades origina-se do uso de combinações impróprias de funções de interpolação para representar os campos de velocidade e pressão. 
Outra dificuldade numérica é a condição de incompressibilidade, onde o campo de velocidade deve manter-se com divergência zero. Desta forma, a pressão deve ser considerada uma variável não relacionada a qualquer equação constitutiva. Sua presença nas equações de balanço de quantidade de movimento tem a finalidade de introduzir um grau de liberdade a mais necessário para satisfazer a condição de divergência zero do campo de velocidades, resultando em um sistema acoplado entre velocidade e pressão.

Uma grande parte dos métodos de resolução numérica de equações diferenciais provém exatamente da formulação variacional do problema, que será discutida a seguir.

\subsection{Formulação Variacional}

Para resolver um problema utilizando o método de elementos finitos deve-se discretizar o domínio no qual se pretende resolver o problema em elementos, os quais podem ser triângulos, quadriláteros, prismas, tetraedros ou outro polígono regular qualquer. Em seguida, é necessário escrever o problema em sua formulação variacional, projetando as equações em um espaço cuja base é conhecida e integrá-las sobre todo domínio. Em outras palavras, deve-se escrever o problema em sua forma variacional ou forma fraca. A seguir, as equações de Navier-Stokes serão obtidas na sua formulação variacional. O desenvolvimento a seguir pode ser encontrado em Sousa, 2005.

\subsubsection{Formulação Variacional das Equações de Navier-Stokes}

Sejam as equações de Navier-Stokes para escoamentos incompressíveis escritas na formulação euleriana, dadas na forma adimensional por

$$
\begin{gathered}
\frac{D(\rho \mathbf{u})}{D t}+\nabla p-\frac{1}{R e} \nabla \cdot\left[\mu\left(\nabla \mathbf{u}+\nabla \mathbf{u}^{\mathbf{T}}\right)\right]-\frac{1}{F r^{2}} \rho \mathbf{g}=0 \\
\nabla \cdot \mathbf{u}=0
\end{gathered}
$$

válidas em um domínio $\Omega \subset \mathbb{R}^{m}$ sujeita às condições de contorno

$$
\begin{gathered}
\mathbf{u}=\mathbf{u}_{\Gamma}, \text { em } \Gamma_{1} \\
u_{t}=0 \text { e } \sigma^{\mathrm{nn}}=0, \text { em } \Gamma_{2} .
\end{gathered}
$$

Considere o subespaço 


$$
\mathbb{V}=H^{1}(\Omega)^{m}=\left\{\mathbf{v}=\left(v_{1}, \ldots, v_{m}\right): v_{i} \in H^{1}(\Omega), \forall i=1, \ldots, m\right\}
$$

onde $H^{1}(\Omega)$ é o espaço de Sobolev dado por

$$
H^{1}(\Omega)=\left\{v \in L^{2}(\Omega): \frac{\partial v}{\partial x_{i}} \in L^{2}(\Omega), i=1, \ldots, m\right\}
$$

com $L^{2}(\Omega)$ sendo um espaço de dimensão infinita caracterizado pela integral de Lesbegue, no entanto, para o caso de funções contínuas, a integral de Lesbegue é equivalente a integral de Riemann e pode ser tratada como

$$
L^{2}(\Omega)=\left\{v: \Omega \rightarrow \mathbb{R}, \int_{\Omega} v^{2} d \Omega<\infty\right\}
$$

$\mathrm{E} \mathbb{V}=H^{1}(\Omega)^{m}$ é o produto cartesiano de $m$ espaços $H^{1}(\Omega)$. Definindo-se

$$
\begin{gathered}
\mathbb{V}_{\mathbf{u} \Gamma}=\mathbf{v} \in \mathbb{V}: \mathbf{v}=\mathbf{u}_{\Gamma} \text { em } \Gamma_{1} \\
\mathbb{P}_{p \Gamma}=q \in L^{2}(\Omega): q=p_{\Gamma} \text { em } \Gamma_{2}
\end{gathered}
$$

a formulação variacional do problema consiste em encontrar soluções $\mathbf{u}(\mathbf{x}, t) \in \mathbb{V}_{\mathbf{u} \Gamma} \mathrm{e}$ $p(\mathbf{x}, t) \in \mathbb{P}_{0}$ tais que

$$
\int_{\Omega}\left\{\frac{D(\rho \mathbf{u})}{D t}+\nabla p-\frac{1}{R e} \nabla \cdot\left[\mu\left(\nabla \mathbf{u}+\nabla \mathbf{u}^{T}\right)\right]-\frac{1}{F r^{2}} \rho \mathbf{g}\right\} \cdot \mathbf{w} d \Omega=0 .
$$

para todo $\mathbf{w} \in \mathbb{V}_{0}$, e

$$
\int_{\Omega}(\nabla \cdot \mathbf{u}) q d \Omega=0
$$

para todo $q \in \mathbb{P}_{0}$. E desenvolvendo a equação 4.10 tem-se

$$
\begin{array}{r}
\int_{\Omega} \frac{D(\rho \mathbf{u})}{D t} \cdot \mathbf{w} d \Omega+\int_{\Omega} \nabla p \cdot \mathbf{w} d \Omega \\
-\int_{\Omega} \frac{1}{R e} \nabla \cdot\left[\mu\left(\nabla \mathbf{u}+\nabla \mathbf{u}^{T}\right)\right] \cdot \mathbf{w} d \Omega-\int_{\Omega} \frac{1}{F r^{2}} \rho \mathbf{g} \cdot \mathbf{w} d \Omega=0 .
\end{array}
$$

$\mathrm{Na}$ integral do termo viscoso aplica-se o teorema de integração por partes de Green resultando 


$$
\begin{aligned}
\int_{\Omega} \nabla \cdot\left[\mu\left(\nabla \mathbf{u}+\nabla \mathbf{u}^{T}\right)\right] \cdot \mathbf{w} d \Omega= & -\int_{\Omega} \mu\left[\left(\nabla \mathbf{u}+\nabla \mathbf{u}^{T}\right): \nabla \mathbf{w}^{T}\right] d \Omega \\
& +\int_{\Gamma} \mathbf{n} \cdot\left[\mu\left(\nabla \mathbf{u}+\nabla \mathbf{u}^{T}\right) \cdot \mathbf{w}\right] d \Gamma
\end{aligned}
$$

onde o operador ':' representa o produto escalar entre dois tensores. A integral no contorno $\Gamma$ pode ser separada em duas integrais, em $\Gamma_{1}$ e $\Gamma_{2}$. A integral em $\Gamma_{1}$ é nula devido ao fato que $\mathbf{w}=\mathbf{0}$ em $\Gamma_{1}$. Em $\Gamma_{2}$ a integral também é nula, o que decorre da condição de contorno (4.4), e portanto, tem-se que a integral em $\Gamma$ é nula. Com a aplicação da integração por partes no termo gradiente de pressão, tem-se

$$
\int_{\Omega} \nabla p \cdot \mathbf{w} d \Omega=-\int_{\Omega} p \nabla \cdot \mathbf{w} d \Omega+\int_{\Gamma} p \mathbf{w} \cdot \mathbf{n} d \Gamma
$$

onde a integral de contorno é nula pois $\mathbf{w}=\mathbf{0}$ em $\Gamma_{1}$ e $p=0$ em $\Gamma_{2}$. Desta forma, obtém-se

$$
\begin{array}{r}
\int_{\Omega} \frac{D(\rho \mathbf{u})}{D t} \cdot \mathbf{w} d \Omega-\int_{\Omega} \nabla p \cdot \mathbf{w} d \Omega \\
-\int_{\Omega} \frac{1}{R e} \nabla \cdot\left[\mu\left(\nabla \mathbf{u}+\nabla \mathbf{u}^{T}\right)\right]: \mathbf{w} d \Omega-\int_{\Omega} \frac{1}{F r^{2}} \rho \mathbf{g} \cdot \mathbf{w} d \Omega=0 .
\end{array}
$$

Definindo-se as formas integrais

$$
\begin{gathered}
m\left(\frac{D \rho \mathbf{u}}{D t}, \mathbf{w}\right)=\int_{\Omega} \frac{D \rho \mathbf{u}}{D t} \cdot \mathbf{w} d \Omega \\
k(\mu, \mathbf{u}, \mathbf{w})=\int_{\Omega} \mu\left[\left(\nabla \mathbf{u}+\nabla \mathbf{u}^{T}\right): \nabla \mathbf{w}^{T}\right] d \Omega \\
g(p, \mathbf{w})=\int_{\Omega} \nabla p \cdot \mathbf{w} d \Omega \\
d(p, \mathbf{w})=\int_{\Omega}(\nabla \cdot \mathbf{w}) p d \Omega
\end{gathered}
$$

Na forma fraca o problema pode ser escrito como segue: Encontrar soluções $\mathbf{u}(\mathbf{x}, t) \in$ $\mathbb{V}_{\mathbf{u} \Gamma}$ e $p(\mathbf{x}, t) \in \mathbb{P}$ tais que

$$
m\left(\frac{D(\rho \mathbf{u})}{D t}, \mathbf{w}\right)-g(p, \mathbf{w})-\frac{1}{R e} k(\mu, \mathbf{u}, \mathbf{w})-\frac{1}{F r^{2}} m(\rho \mathbf{g}, \mathbf{w})=0
$$




$$
d(q, \mathbf{u})=0
$$

para todo $\mathbf{w} \in \mathbb{V}_{0}$ e $q \in \mathbb{P}_{0}$.

\subsection{O Método de Elementos Finitos}

\subsubsection{Considerações Iniciais}

O método dos elementos finitos é uma técnica genérica para construir soluções aproximadas para problemas de valores de contorno. O método envolve dividir o domínio da solução em um número finito de simples subdomínios, os elementos finitos, e usar "conceitos variacionais" para aproximação da solução, através da coleção dos elementos finitos Becker et al., 1981. Tais elementos são compostos por pontos nodais sobre os quais é equacionado o sistema de equações algébricas resultante.

Os denominados elementos finitos são pequenas porções do domínio físico do problema, onde a variação das incógnitas do problema no interior de tais elementos é aproximada através da aplicação das chamadas funções de interpolação. Estabelece-se, então, uma sentença de resíduos ponderados, afim de proporcionar uma distribuição do erro envolvido em tal aproximação ao longo de todos os elementos finitos que compõem o domínio físico do problema, através do uso de funções auxiliares ou de ponderação, que compõem o núcleo das integrais.

Tal método foi gerado com o intuito de melhor representar problemas possuindo domínios físicos contendo uma geometria intrincada e de forma a simplificar as aplicações das condições de contorno associadas, eliminando assim algumas das dificuldades do método de diferenças finitas.

A implementação computacional do método de elementos finitos consiste na montagem de sub-matrizes que computam as propriedades de cada elemento, através de coeficientes de influência, para então se formar o sistema de equações algébricas associado ao domínio físico do problema, isto é, ao conjunto de elementos utilizados para a discretização. Neste trabalho o domínio será discretizado por elementos prismáticos lineares.

Ao invés de buscar uma função aproximadora que satisfaça as condições de contorno para todo o domínio, no método de elementos finitos as funções admissíveis são definidas no domínio de cada elemento finito. Neste caso, o domínio $\Omega$ é discretizado por um número 
finito de elementos $\Omega_{e}$ de forma que

$$
\Omega=\bigcup_{e=1}^{M} \Omega_{e}
$$

Considere uma função $\phi: \mathbb{R}^{m} \rightarrow \mathbb{R}$ como sendo a solução exata de um determinado problema, válida num domínio $\Omega$ com condições de contorno estabelecidas. A idéia geral do método de elementos finitos é encontrar uma aproximação $\Phi(\mathbf{x})$ da solução exata $\phi(x)$ de forma particionada em cada elemento $\Omega_{e}$ do domínio, isto é, a aproximação de $\phi(x)$ restrita a cada elemento é nula para qualquer ponto $\mathrm{x}$ fora de $\Omega_{e}$. Logo a função aproximadora de $\phi(x)$ é dada por

$$
\Phi=\sum_{e} \phi^{e}(\mathbf{x})
$$

Como o valor de $\phi$ nos vértices da malha que discretiza o domínio $\Omega$ é chamado de valor nodal de $\phi$, então pode-se denotá-lo por $\phi_{i}$, onde $i$ é o número do nó correspondente,

$$
\phi_{i}=\phi(x)
$$

Seja um elemento poligonal com $s$ lados, e considere associado a cada vértice do elemento o valor de $\phi$, com $\phi_{i}=1, \ldots, s$. A cada elemento pode ser associada uma gama de funções de interpolação, representada genericamente pela matriz

$$
\psi^{e}(x)=\left[\begin{array}{ccc}
\psi_{1,1}^{e}(\mathbf{x}) & \ldots & \psi_{1, s}^{e}(\mathbf{x}) \\
\vdots & & \vdots \\
\psi_{r, 1}^{e}(\mathbf{x}) & \ldots & \psi_{r, s}^{e}(\mathbf{x})
\end{array}\right]
$$

onde $r$ representa o grau de liberdade de cada nó, e $s$ representa a quantidade de nós em cada elemento. Desta forma, o número de graus de liberdade no elemento é dado por $r \cdot s$, pois o número de graus de liberdade associado a um vértice é a quantidade de variáveis relacionadas com este mesmo vértice, e o número de graus de liberdade de um elemento é a soma dos graus de liberdade dos vértices que definem tal elemento.

Assumindo que cada nó tenha somente um grau de liberdade, tem-se

$$
\psi^{e}(x)=\left[\psi_{1}^{e}(\mathbf{x}), \ldots, \psi_{s}^{e}(\mathbf{x})\right]
$$


Logo, sendo

$$
\delta^{e}=\left[\phi_{1}, \ldots, \phi_{s}\right]^{T}
$$

A aproximação para $\phi$ em cada elemento é dada por

$$
\phi^{e}(\mathbf{x})=\psi^{e} \delta^{e}
$$

e a função aproximadora 4.23 pode ser escrita como

$$
\Phi=\sum_{e=1}^{N E} \phi^{e}(\mathbf{x})=\sum_{e=1}^{N E} \psi^{e} \delta^{e}
$$

onde $N E$ é o número total de elementos.

\subsubsection{Funções de Interpolação}

Após discretizar o domínio, é necessário escolher como são as funções de interpolação que são conhecidas como funções de forma. Uma escolha típica é por funções polinomiais. A melhor forma de se obter tais funções é através de interpolação direta no elemento. A malha utilizada pelo método de elementos finitos possibilita grande variedade de escolha dos elementos. Estes são classificados por sua geometria, podendo ser triangulares e retangulares para o caso em duas dimensões, tetraedrais, hexaedrais e prismáticos para o caso em três dimensões e pelo tipo de interpolação empregada que varia com a ordem do polinômio interpolador, como linear, quadrática, bilinear, cúbica, etc.

Nesta seção são apresentadas algumas funções de interpolação para elementos lineares, triangulares e prismáticos.

\section{Funções de Interpolação de Lagrange para Elementos Lineares}

Aqui será mostrada uma técnica para gerar funções de forma polinomiais de qualquer grau $k$, que pode ser construída como segue. Primeiramente considera-se um típico elemento finito $\Omega_{e}$, onde considera-se um sistema de coordenadas local $\xi$, com a origem no centro do elemento, com escala no domínio de $[-1,1]$. Essa escala pode ser conseguida através de uma simples transformação linear. Todos os cálculos dos elementos são centrados neste elemento "mestre", onde a função de forma no elemento mestre é denotada por $\hat{\psi}(\xi)$. 
Para funções de forma de grau $k$, tem-se a forma geral:

$$
\hat{\psi}_{i}(\xi)=\frac{\left(\xi-\xi_{1}\right)\left(\xi-\xi_{2}\right) \ldots\left(\xi-\xi_{i-1}\right)\left(\xi-\xi_{i+1}\right) \ldots\left(\xi-\xi_{k+1}\right)}{\left(\xi_{i}-\xi_{1}\right)\left(\xi_{i}-\xi_{2}\right) \ldots\left(\xi_{i}-\xi_{i-1}\right)\left(\xi_{i}-\xi_{i+1}\right) \ldots\left(\xi_{i}-\xi_{k+1}\right)}
$$

Note que

$$
\hat{\psi}_{i}\left(\xi_{j}\right)= \begin{cases}1 & \text { se } i=j \\ 0 & \text { se } i \neq j\end{cases}
$$

o que implica que $\hat{\psi}_{i}(\xi)$ são linearmente independentes.

Assim, para $k=1$, tem-se as funções de forma lineares com dois nós e estas são dadas por:

$$
\begin{aligned}
& \hat{\psi}_{1}(\xi)=\frac{\xi-\xi_{2}}{\xi_{1}-\xi_{2}}=\frac{1}{2}(1-\xi) \\
& \hat{\psi}_{2}(\xi)=\frac{\xi-\xi_{1}}{\xi_{2}-\xi_{1}}=\frac{1}{2}(1+\xi)
\end{aligned}
$$

\section{Funções de Interpolação para Elementos Triangulares}

Para elementos deste tipo, considera-se triângulos que tenham os lados retos. O mapeamento de um triângulo isósceles (Figura 4.1) como elemento mestre é linear, facilitando o mapeamento e a formulação das matrizes do elemento. Assim, as três funções de forma lineares para um elemento mestre formado por um triângulo isósceles são:

$$
\begin{gathered}
\widehat{\psi}_{1}(\xi, \eta)=1-\xi-\eta \\
\widehat{\psi}_{2}(\xi, \eta)=\xi \\
\widehat{\psi}_{3}(\xi, \eta)=\eta
\end{gathered}
$$

para os nós 1, 2 e 3 respectivamente.

Mesmo sendo possível a determinação de funções de interpolação em coordenadas globais para o triângulo, o uso de coordenadas locais simplifica os cálculos no momento de resolução das integrais do modelo variacional. Considere um sistema de coordenadas locais no triângulo $\left(L_{1}, L_{2}, L_{3}\right)$ dado por

$$
L_{1}=\frac{A_{1}}{A}, L_{2}=\frac{A_{2}}{A}, L_{3}=\frac{A_{3}}{A}
$$

onde $A=A_{1}+A_{2}+A_{3}$ é a área do triângulo e $A_{1}, A_{2}$ e $A_{3}$ são as áreas mostradas na Figura 


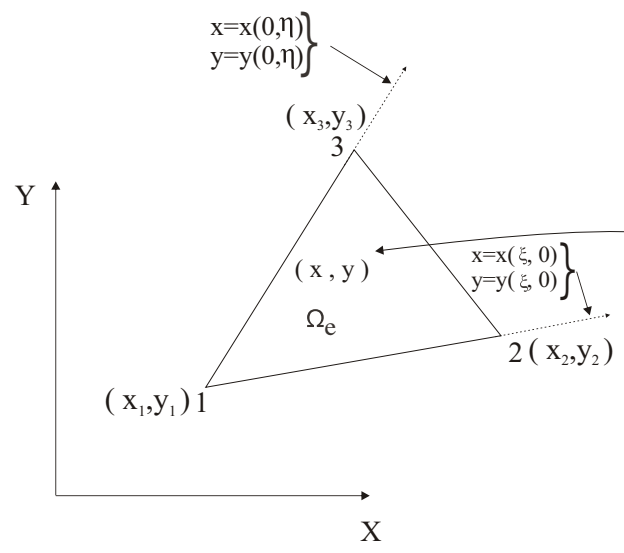

Figura 4.1: Mapeamento linear do triângulo $\Omega_{e}$ para o elemento mestre $\widehat{\Omega}$ e o mapeamento inverso.

4.2. Observe que qualquer ponto sobre a aresta entre os vértices 1 e 2 tem coordenada $L_{3}=0$, e se o ponto coincide com o vértice 3 , então $L_{3}=1$, uma vez que $A_{3}=A$. Assim, qualquer ponto dentro do triângulo pode ser representada pelas coordenadas $\left(L_{1}, L_{2}, L_{3}\right)$, também chamadas de coordenadas baricêntricas. Note que

$$
L_{1}+L_{2}+L_{3}=1
$$

Tem-se que a relação entre o sistema de coordenadas global $(x, y)$ e o sistema de coordenadas local $\left(L_{1}, L_{2}, L_{3}\right)$ é dada por

$$
\begin{gathered}
x=L_{1} x_{1}+L_{2} x_{2}+L_{3} x_{3}, \\
y=L_{1} y_{1}+L_{2} y_{2}+L_{3} y_{3}
\end{gathered}
$$

As equações (4.38), 4.39) e 4.40 podem ser escritas na forma matricial como

$$
\left[\begin{array}{l}
1 \\
x \\
y
\end{array}\right]=\left[\begin{array}{ccc}
1 & 1 & 1 \\
x_{1} & x_{2} & x_{3} \\
y_{1} & y_{2} & y_{3}
\end{array}\right]\left[\begin{array}{l}
L_{1} \\
L_{2} \\
L_{3}
\end{array}\right]
$$

Pode-se inverter (4.41) e obter 


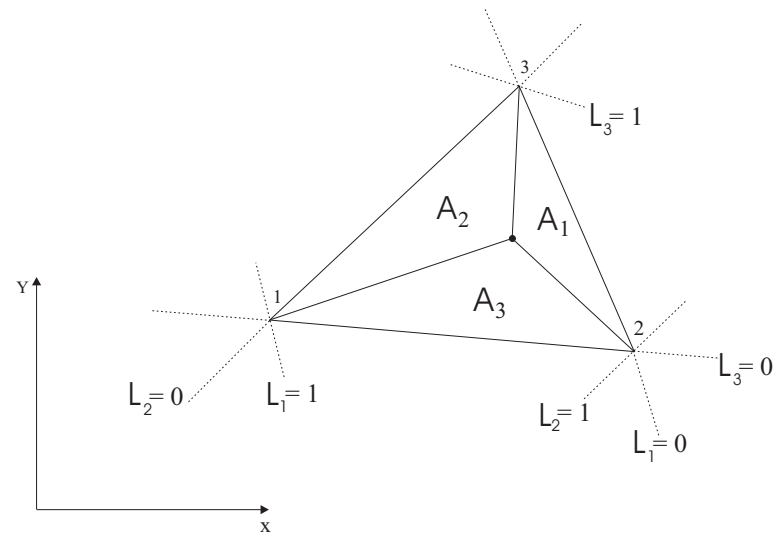

Figura 4.2: Determinação de funções de interpolação para um elemento triangular.

$$
\left[\begin{array}{l}
L_{1} \\
L_{2} \\
L_{3}
\end{array}\right]=\frac{1}{2 A}\left[\begin{array}{lll}
\left(x_{2} y_{3}-x_{3} y_{2}\right) & \left(y_{2}-y_{3}\right) & \left(x_{3}-x_{2}\right) \\
\left(x_{3} y_{1}-x_{1} y_{3}\right) & \left(y_{3}-y_{1}\right) & \left(x_{1}-x_{3}\right) \\
\left(x_{1} y_{2}-x_{2} y_{1}\right) & \left(y_{1}-y_{2}\right) & \left(x_{2}-x_{1}\right)
\end{array}\right]\left[\begin{array}{l}
1 \\
x \\
y
\end{array}\right]
$$

onde a área $A$ é a área do triângulo dada por

$$
A=\frac{1}{2}\left|\begin{array}{ccc}
1 & x_{1} & y_{1} \\
1 & x_{2} & y_{2} \\
1 & x_{3} & y_{3}
\end{array}\right|
$$

De 4.42 as seguintes relações para as derivadas podem ser obtidas

$$
\begin{array}{ll}
\frac{\partial L_{1}}{\partial x}=\frac{y_{2}-y_{3}}{2 A}, & \frac{\partial L_{1}}{\partial y}=\frac{x_{3}-x_{2}}{2 A} . \\
\frac{\partial L_{2}}{\partial x}=\frac{y_{3}-y_{1}}{2 A}, & \frac{\partial L_{2}}{\partial y}=\frac{x_{1}-x_{3}}{2 A} . \\
\frac{\partial L_{3}}{\partial x}=\frac{y_{1}-y_{2}}{2 A}, & \frac{\partial L_{3}}{\partial y}=\frac{x_{2}-x_{1}}{2 A} .
\end{array}
$$

Um resultado sobre integração em coordenadas de área para a discretização por elementos triangulares é dado por

$$
\int_{A} L_{1}^{m} L_{2}^{n} L_{3}^{p} d x d y=\frac{m ! n ! p !}{(m+n+p+2) !} 2 A
$$


A desmontração da expressão 4.47) pode ser encontrada em Davies, 1980.

Seja um elemento triangular com um grau de liberdade em cada nó, a aproximação da variável $\phi$ no elemento é dado por

$$
\phi^{e}(x, y)=\alpha_{0}+\alpha_{1} x+\alpha_{2} y
$$

e fazendo-se uso das coordenadas baricêntricas do triângulo $e$ como funções de interpolação, isto é,

$$
\psi^{e}=\left[\begin{array}{lll}
L_{1} & L_{2} & L_{3}
\end{array}\right]
$$

tem-se que

$$
\phi^{e}(x, y)=\psi^{e}(x, y) \delta^{e}=\left[\begin{array}{lll}
L_{1}(x, y) & L_{2}(x, y) & L_{3}(x, y)
\end{array}\right]\left[\begin{array}{l}
\phi_{1} \\
\phi_{2} \\
\phi_{3}
\end{array}\right]
$$

\section{Funções de Interpolação para Elementos Prismáticos}

As funções de forma para um elemento prismático linear são mostradas na Figura 4.3 . Note que cada função de forma é definida para cada vértice do elemento, numerados na figura de 1 a 6 . As funções de forma são especificadas em termos de coordenadas locais.

E as derivadas são dadas por:

$$
\begin{aligned}
& \frac{\partial \psi_{1}}{\partial \xi}=-\frac{1}{2}(1-\zeta) \quad \frac{\partial \psi_{1}}{\partial \eta}=-\frac{1}{2}(1-\zeta) \quad \frac{\partial \psi_{1}}{\partial \zeta}=-\frac{1}{2}(1-\xi-\eta) \\
& \frac{\partial \psi_{2}}{\partial \xi}=\frac{1}{2}(1-\zeta) \quad \frac{\partial \psi_{2}}{\partial \eta}=0 \quad \frac{\partial \psi_{2}}{\partial \zeta}=-\frac{1}{2} \xi \\
& \frac{\partial \psi_{3}}{\partial \xi}=0 \quad \frac{\partial \psi_{3}}{\partial \eta}=\frac{1}{2}(1-\zeta) \quad \frac{\partial \psi_{3}}{\partial \zeta}=-\frac{1}{2} \eta \\
& \frac{\partial \psi_{4}}{\partial \xi}=-\frac{1}{2}(1+\zeta) \quad \frac{\partial \psi_{4}}{\partial \eta}=-\frac{1}{2}(1+\zeta) \quad \frac{\partial \psi_{4}}{\partial \zeta}=\frac{1}{2}(1-\xi-\eta) \\
& \frac{\partial \psi_{5}}{\partial \xi}=\frac{1}{2}(1+\zeta) \quad \frac{\partial \psi_{5}}{\partial \eta}=0 \quad \frac{\partial \psi_{5}}{\partial \zeta}=\frac{1}{2} \xi \\
& \frac{\partial \psi_{6}}{\partial \xi}=0 \quad \frac{\partial \psi_{6}}{\partial \eta}=\frac{1}{2}(1+\zeta) \quad \frac{\partial \psi_{6}}{\partial \zeta}=\frac{1}{2} \eta
\end{aligned}
$$




$$
\begin{aligned}
& \psi_{1}(\xi, \eta, \zeta)=\frac{1}{2}(1-\xi-\eta)(1-\zeta) \\
& \psi_{2}(\xi, \eta, \zeta)=\frac{1}{2} \xi(1-\zeta) \\
& \psi_{3}(\xi, \eta, \zeta)=\frac{1}{2} \eta(1-\zeta) \\
& \psi_{4}(\xi, \eta, \zeta)=\frac{1}{2}(1-\xi-\eta)(1+\zeta) \\
& \psi_{5}(\xi, \eta, \zeta)=\frac{1}{2} \xi(1+\zeta) \\
& \psi_{6}(\xi, \eta, \zeta)=\frac{1}{2} \eta(1+\zeta)
\end{aligned}
$$

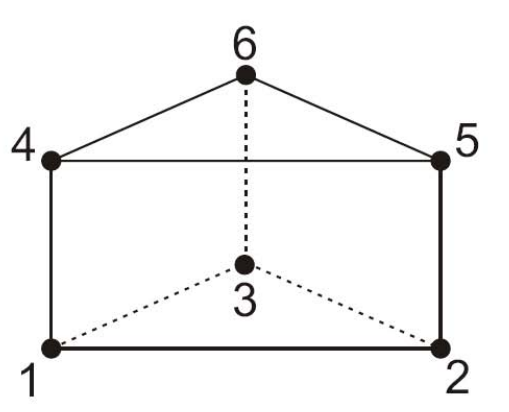

Figura 4.3: Funções de forma para elementos prismáticos lineares.

\subsection{Discretização Espacial}

Até aqui as equações de Navier-Stokes foram escritas na sua formulação variacional, porém na forma contínua. O objetivo desta seção é discretizá-las no espaço, de forma a obter um sistema de equações diferenciais ordinárias. As equações são discretizadas apenas no domínio espacial, permanecendo contínuas no domínio do tempo.

\subsubsection{Método de Galerkin para as Equações de Navier-Stokes}

Sejam as equações de Navier-Stokes em sua forma adimensional, dadas em 3.20) e (3.21). Considere estas equações desenvolvidas em um sistema cartesiano tridimensional. Sejam

$$
\mathbf{u}=(u, v, w), \quad \mathbf{g}=\left(g_{x}, g_{y}, g_{z}\right), \quad \mathbf{w}=\left(w_{x}, w_{y}, w_{z}\right)
$$

A equação 4.20 torna-se

$$
\int_{\Omega}\left(\frac{D(\rho u)}{D t} w_{x}+\frac{D(\rho v)}{D t} w_{y}+\frac{D(\rho v)}{D t} w_{z}\right) d \Omega
$$




$$
\begin{gathered}
-\int_{\Omega}\left(p \frac{\partial w_{x}}{\partial x}+p \frac{\partial w_{y}}{\partial y}+p \frac{\partial w_{z}}{\partial z}\right) d \Omega-\frac{1}{R e} \int_{\Omega} \mu\left[\left(\frac{\partial u}{\partial x} \frac{\partial w_{x}}{\partial x}+\frac{\partial v}{\partial x} \frac{\partial w_{y}}{\partial x}+\frac{\partial w}{\partial x} \frac{\partial w_{z}}{\partial x}+\right.\right. \\
\left.\frac{\partial u}{\partial y} \frac{\partial w_{x}}{\partial y}+\frac{\partial v}{\partial y} \frac{\partial w_{y}}{\partial y}+\frac{\partial w}{\partial y} \frac{\partial w_{z}}{\partial y}+\frac{\partial u}{\partial z} \frac{\partial w_{x}}{\partial z}+\frac{\partial v}{\partial z} \frac{\partial w_{y}}{\partial z}+\frac{\partial w}{\partial z} \frac{\partial w_{z}}{\partial z}\right)+ \\
+\left(\frac{\partial u}{\partial x} \frac{\partial w_{x}}{\partial x}+\frac{\partial u}{\partial y} \frac{\partial w_{y}}{\partial x}+\frac{\partial u}{\partial z} \frac{\partial w_{z}}{\partial x}+\frac{\partial v}{\partial x} \frac{\partial w_{x}}{\partial y}+\frac{\partial v}{\partial y} \frac{\partial w_{y}}{\partial y}+\frac{\partial v}{\partial z} \frac{\partial w_{z}}{\partial y}+\frac{\partial w}{\partial x} \frac{\partial w_{x}}{\partial z}+\right. \\
\left.\left.\frac{\partial w}{\partial y} \frac{\partial w_{y}}{\partial z}+\frac{\partial w}{\partial z} \frac{\partial w_{z}}{\partial z}\right)\right] d \Omega-\frac{1}{F r^{2}} \int_{\Omega} \rho\left(g_{x} w_{x}+g_{y} w_{y}+g_{z} w_{z}\right) d \Omega=0
\end{gathered}
$$

Pela formulação variacional do problema é necessário determinar soluções $\mathbf{u}=$ $(u, v, w) \in \mathbb{V}_{u \Gamma}$ e $p \in \mathbb{P}$ de modo que (4.52) seja verdadeira para todo $\mathbf{w}=\left(w_{x}, w_{y}, w_{z}\right) \in$ $\mathbb{V}_{0}$.

No entanto, sendo satisfeitas as expressões

$$
\begin{aligned}
\int_{\Omega} \frac{D(\rho u)}{D t} w_{x} \Omega- & \int_{\Omega} p \frac{\partial w_{x}}{\partial x} d \Omega-\frac{1}{R e} \int_{\Omega} \mu\left[\left(\frac{\partial u}{\partial x} \frac{\partial w_{x}}{\partial x}+\frac{\partial u}{\partial y} \frac{\partial w_{x}}{\partial y}+\frac{\partial u}{\partial z} \frac{\partial w_{x}}{\partial z}+\right.\right. \\
& \left.\left.\frac{\partial u}{\partial x} \frac{\partial w_{x}}{\partial x}+\frac{\partial v}{\partial x} \frac{\partial w_{x}}{\partial y}+\frac{\partial w}{\partial x} \frac{\partial w_{x}}{\partial z}+\right)\right] d \Omega=0 \\
\int_{\Omega} \frac{D(\rho v)}{D t} w_{y} \Omega- & \int_{\Omega} p \frac{\partial w_{y}}{\partial y} d \Omega-\frac{1}{R e} \int_{\Omega} \mu\left[\left(\frac{\partial v}{\partial x} \frac{\partial w_{y}}{\partial x}+\frac{\partial v}{\partial y} \frac{\partial w_{y}}{\partial y}+\frac{\partial v}{\partial z} \frac{\partial w_{y}}{\partial z}+\right.\right. \\
& \left.\left.\frac{\partial u}{\partial y} \frac{\partial w_{y}}{\partial x}+\frac{\partial v}{\partial y} \frac{\partial w_{y}}{\partial y}+\frac{\partial w}{\partial y} \frac{\partial w_{y}}{\partial z}+\right)\right] d \Omega=0 \\
\int_{\Omega} \frac{D(\rho w)}{D t} w_{z} \Omega- & \int_{\Omega} p \frac{\partial w_{z}}{\partial z} d \Omega-\frac{1}{R e} \int_{\Omega} \mu\left[\left(\frac{\partial w}{\partial x} \frac{\partial w_{z}}{\partial x}+\frac{\partial v}{\partial y} \frac{\partial w_{z}}{\partial y}+\frac{\partial v}{\partial z} \frac{\partial w_{z}}{\partial z}+\right.\right. \\
& \left.\left.\frac{\partial u}{\partial z} \frac{\partial w_{z}}{\partial x}+\frac{\partial v}{\partial z} \frac{\partial w_{z}}{\partial y}+\frac{\partial w}{\partial z} \frac{\partial w_{z}}{\partial z}+\right)\right] d \Omega=0
\end{aligned}
$$

para quaisquer $w_{x} \in \mathbb{V}_{0}, w_{y} \in \mathbb{V}_{0}$ e $w_{z} \in \mathbb{V}_{0}$, respectivamente, então 4.52 é satisfeita. Assim pode-se trabalhar com as equações nas direções $x, y$ e na direção $z$ separadamente, sem que haja alguma perda de generalidade.

Para a equação 4.21, tem-se

$$
\int_{\Omega}\left(\frac{\partial u}{\partial x}+\frac{\partial v}{\partial y}+\frac{\partial w}{\partial z}\right) q d \Omega=0
$$

Considere $N V$ o número de pontos de velocidade, $N P$ o número de pontos de pressão e 
$N E$ o número de elementos finitos na malha de elementos finitos que discretiza o domínio $\Omega$. O método de Galerkin consiste em fazer as seguintes substituições em 4.52

$$
\begin{aligned}
u(\mathbf{x}, t) & \approx \sum_{n=1}^{N V} \psi_{n}(\mathbf{x}) u_{n}(t) \\
v(\mathbf{x}, t) & \approx \sum_{n=1}^{N V} \psi_{n}(\mathbf{x}) v_{n}(t) \\
w(\mathbf{x}, t) & \approx \sum_{n=1}^{N V} \psi_{n}(\mathbf{x}) w_{n}(t) \\
p(\mathbf{x}, t) & \approx \sum_{n=1}^{N P} P_{r}(\mathbf{x}) p_{r}(t)
\end{aligned}
$$

que são aproximações semi-contínuas, isto é, contínuas no tempo $(t)$ e discretas no espaço $(x)$. Aqui, $\psi_{n}(x)$ representam as funções de interpolação utilizadas para a velocidade e $P_{n}(x)$ as funções de interpolação para a pressão. Considere ainda:

$$
\begin{aligned}
g_{x}(\mathbf{x}, t) & \approx \sum_{n=1}^{N V} \psi_{n}(\mathbf{x}) g_{x, n}(t) \\
g_{y}(\mathbf{x}, t) & \approx \sum_{n=1}^{N V} \psi_{n}(\mathbf{x}) g_{y, n}(t) \\
g_{z}(\mathbf{x}, t) & \approx \sum_{n=1}^{N V} \psi_{n}(\mathbf{x}) g_{z, n}(t)
\end{aligned}
$$

como sendo as aproximações para os termos de gravidade, e

$$
\begin{aligned}
& \rho(\mathbf{x}, t) \approx \sum_{e=1}^{N E} \rho^{e}(t) \\
& \mu(\mathbf{x}, t) \approx \sum_{e=1}^{N E} \mu^{e}(t)
\end{aligned}
$$

como sendo as aproximações para as propriedades do fluido.

A equação de balanço da quantidade de movimento é normalmente avaliada em todos os nós livres de velocidade, e portanto as funções peso $w_{x}, w_{y}$ e $w_{z}$ são substituídas por 
funções de interpolação $\psi_{m}=\psi_{m}(x), m=1, \ldots, N V$. Aplicando este procedimento para as direções $x, y$ e $z$, e restringindo-se as funções de interpolação nodais a cada elemento $e$, na direção $x$, tem-se:

$$
\begin{gathered}
\sum_{e} \int_{\Omega_{e}} \sum_{i, j \in e} \rho^{e} \frac{D u_{j}}{D t} \psi_{i}^{e} \psi_{j}^{e} d \Omega-\sum_{e} \int_{\Omega_{e}} \sum_{i, k \in e} \frac{\partial \psi_{i}^{e}}{\partial x} P_{k}^{e} p_{k} d \Omega \\
-\frac{1}{R e} \sum_{e} \int_{\Omega_{e}} \sum_{i, j \in e} \mu^{e}\left(\frac{\partial \psi_{i}^{e}}{\partial x} \frac{\partial \psi_{j}^{e}}{\partial x} u_{j}+\frac{\partial \psi_{i}^{e}}{\partial y} \frac{\partial \psi_{j}^{e}}{\partial y} u_{j}+\frac{\partial \psi_{i}^{e}}{\partial z} \frac{\partial \psi_{j}^{e}}{\partial z} u_{j}+\frac{\partial \psi_{i}^{e}}{\partial x} \frac{\partial \psi_{j}^{e}}{\partial x} u_{j}+\right. \\
\left.+\frac{\partial \psi_{i}^{e}}{\partial y} \frac{\partial \psi_{j}^{e}}{\partial x} v_{j}+\frac{\partial \psi_{i}^{e}}{\partial z} \frac{\partial \psi_{j}^{e}}{\partial x} w_{j}\right) d \Omega-\frac{1}{F r^{2}} \sum_{e} \int_{\Omega_{e}} \sum_{i, j \in e} \rho^{e} \psi_{i}^{e} \psi_{j}^{e} g_{x, j} d \Omega=0
\end{gathered}
$$

Na direção $y$,

$$
\begin{gathered}
\sum_{e} \int_{\Omega_{e}} \sum_{i, j \in e} \rho^{e} \frac{D v_{j}}{D t} \psi_{i}^{e} \psi_{j}^{e} d \Omega-\sum_{e} \int_{\Omega_{e}} \sum_{i, k \in e} \frac{\partial \psi_{i}^{e}}{\partial y} P_{k}^{e} p_{k} d \Omega \\
-\frac{1}{R e} \sum_{e} \int_{\Omega_{e}} \sum_{i, j \in e} \mu^{e}\left(\frac{\partial \psi_{i}^{e}}{\partial x} \frac{\partial \psi_{j}^{e}}{\partial x} v_{j}+\frac{\partial \psi_{i}^{e}}{\partial y} \frac{\partial \psi_{j}^{e}}{\partial y} v_{j}+\frac{\partial \psi_{i}^{e}}{\partial z} \frac{\partial \psi_{j}^{e}}{\partial z} v_{j}+\frac{\partial \psi_{i}^{e}}{\partial x} \frac{\partial \psi_{j}^{e}}{\partial y} u_{j}+\right. \\
\left.+\frac{\partial \psi_{i}^{e}}{\partial y} \frac{\partial \psi_{j}^{e}}{\partial y} v_{j}+\frac{\partial \psi_{i}^{e}}{\partial z} \frac{\partial \psi_{j}^{e}}{\partial y} w_{j}\right) d \Omega-\frac{1}{F r^{2}} \sum_{e} \int_{\Omega_{e}} \sum_{i, j \in e} \rho^{e} \psi_{i}^{e} \psi_{j}^{e} g_{y, j} d \Omega=0
\end{gathered}
$$

$\mathrm{Na}$ direção $z$,

$$
\begin{gathered}
\sum_{e} \int_{\Omega_{e}} \sum_{i, j \in e} \rho^{e} \frac{D w_{j}}{D t} \psi_{i}^{e} \psi_{j}^{e} d \Omega-\sum_{e} \int_{\Omega_{e}} \sum_{i, k \in e} \frac{\partial \psi_{i}^{e}}{\partial z} P_{k}^{e} p_{k} d \Omega \\
-\frac{1}{R e} \sum_{e} \int_{\Omega_{e}} \sum_{i, j \in e} \mu^{e}\left(\frac{\partial \psi_{i}^{e}}{\partial x} \frac{\partial \psi_{j}^{e}}{\partial x} w_{j}+\frac{\partial \psi_{i}^{e}}{\partial y} \frac{\partial \psi_{j}^{e}}{\partial y} w_{j}+\frac{\partial \psi_{i}^{e}}{\partial z} \frac{\partial \psi_{j}^{e}}{\partial z} w_{j}+\frac{\partial \psi_{i}^{e}}{\partial x} \frac{\partial \psi_{j}^{e}}{\partial z} u_{j}+\right. \\
\left.+\frac{\partial \psi_{i}^{e}}{\partial y} \frac{\partial \psi_{j}^{e}}{\partial z} v_{j}+\frac{\partial \psi_{i}^{e}}{\partial z} \frac{\partial \psi_{j}^{e}}{\partial z} w_{j}\right) d \Omega-\frac{1}{F r^{2}} \sum_{e} \int_{\Omega_{e}} \sum_{i, j \in e} \rho^{e} \psi_{i}^{e} \psi_{j}^{e} g_{z, j} d \Omega=0
\end{gathered}
$$

A equação da continuidade 4.56), é avaliada nos nós livres de pressão, e portanto, a função peso $q$ é aproximada pelas funções de interpolação associadas à pressão $P_{r}(x)$, resultando

$$
\sum_{e} \int_{\Omega^{e}} \sum_{n}\left(\frac{\partial \psi_{n}}{\partial x} u_{n}+\frac{\partial \psi_{n}}{\partial y} v_{n}+\frac{\partial \psi_{n}}{\partial z} w_{n}\right) P_{r} d \Omega=0
$$


para $r=1, \ldots, N P$. Restringindo as funções de interpolação a cada elemento $e$, vem

$$
\sum_{e} \int_{\Omega^{e}} \sum_{j, k \in e}\left(\frac{\partial \psi_{j}^{e}}{\partial x} u_{j}+\frac{\partial \psi_{j}^{e}}{\partial y} v_{j}+\frac{\partial \psi_{j}^{e}}{\partial z} w_{j}\right) P_{k}^{e} d \Omega=0
$$

As equações 4.66), 4.67) e 4.68 podem ser representadas na forma de um sistema de equações diferenciais ordinárias

$$
\begin{gathered}
\mathbf{M}_{\rho, x} \frac{D u}{D t}-\frac{1}{R e}\left(\left(2 \mathbf{K}_{x x}+\mathbf{K}_{y y}+\mathbf{K}_{z z}\right) \mathbf{u}+\mathbf{K}_{x y} \mathbf{v}+\mathbf{K}_{x z} \mathbf{w}\right)-\mathbf{G}_{x} \mathbf{p}-\frac{1}{F r^{2}} \mathbf{M}_{\rho, x} \mathbf{g}_{x}=0 \\
\mathbf{M}_{\rho, y} \frac{D v}{D t}-\frac{1}{R e}\left(\mathbf{K}_{y x} \mathbf{u}+\left(\mathbf{K}_{x x}+2 \mathbf{K}_{y y}+\mathbf{K}_{z z}\right) \mathbf{v}+\mathbf{K}_{y z} \mathbf{w}\right)-\mathbf{G}_{y} \mathbf{p}-\frac{1}{F r^{2}} \mathbf{M}_{\rho, y} \mathbf{g}_{y}=0 \\
\mathbf{M}_{\rho, z} \frac{D w}{D t}-\frac{1}{R e}\left(\mathbf{K}_{z x} \mathbf{u}+\mathbf{K}_{z y} \mathbf{v}+\left(\mathbf{K}_{x x}+\mathbf{K}_{y y}+2 \mathbf{K}_{z z}\right) \mathbf{w}+\right)-\mathbf{G}_{z} \mathbf{p}-\frac{1}{F r^{2}} \mathbf{M}_{\rho, z} \mathbf{g}_{z}=0 \\
\mathbf{D}_{x} \mathbf{u}+\mathbf{D}_{y} \mathbf{v}+\mathbf{D}_{z} \mathbf{w}=0
\end{gathered}
$$

onde $\mathbf{u}=\left[u_{1}, \ldots, u_{N U}\right]^{T}, \mathbf{v}=\left[v_{1}, \ldots, v_{N V}\right]^{T}, \mathbf{w}=\left[w_{1}, \ldots, w_{N U}\right]^{T} \mathbf{p}=\left[p_{1}, \ldots, p_{N V}\right]^{T}$, $\mathbf{g}_{x}=\left[g_{1}^{x}, \ldots, g_{N V}^{x}\right]^{T}, \mathbf{g}_{y}=\left[g_{1}^{y}, \ldots, g_{N V}^{y}\right]^{T}, \mathbf{g}_{z}=\left[g_{1}^{z}, \ldots, g_{N V}^{z}\right]^{T}$ são os vetores dos valores nodais para as variáveis de velocidade e pressão, e as forças de gravidade. As matrizes deste sistema de EDO's são dadas por

$$
\begin{aligned}
& \mathbf{M}_{\rho, x}=\mathcal{A}_{x}\left(\mathbf{m}_{\rho}^{e}\right), \quad \mathbf{M}_{\rho, y}=\mathcal{A}_{y}\left(\mathbf{m}_{\rho}^{e}\right), \quad \mathbf{M}_{\rho, z}=\mathcal{A}_{z}\left(\mathbf{m}_{\rho}^{e}\right), \\
& \mathbf{K}_{x, x}=\mathcal{A}_{x}\left(\mathbf{k}_{x x}^{e}\right), \quad \mathbf{K}_{x, y}=\mathcal{A}_{x}\left(\mathbf{k}_{x y}^{e}\right), \quad \mathbf{K}_{x, z}=\mathcal{A}_{x}\left(\mathbf{k}_{x z}^{e}\right), \\
& \mathbf{K}_{y, x}=\mathcal{A}_{y}\left(\mathbf{k}_{y x}^{e}\right), \quad \mathbf{K}_{y, y}=\mathcal{A}_{y}\left(\mathbf{k}_{y y}^{e}\right), \quad \mathbf{K}_{y, z}=\mathcal{A}_{y}\left(\mathbf{k}_{y z}^{e}\right), \\
& \mathbf{K}_{z, x}=\mathcal{A}_{z}\left(\mathbf{k}_{z x}^{e}\right), \quad \mathbf{K}_{z, y}=\mathcal{A}_{z}\left(\mathbf{k}_{z y}^{e}\right), \quad \mathbf{K}_{z, z}=\mathcal{A}_{z}\left(\mathbf{k}_{z z}^{e}\right), \\
& \mathbf{G}_{x}=\mathcal{A}_{x}\left(\mathbf{g}_{x}^{e}\right), \quad \mathbf{G}_{y}=\mathcal{A}_{y}\left(\mathbf{g}_{y}^{e}\right), \quad \mathbf{G}_{z}=\mathcal{A}_{z}\left(\mathbf{g}_{z}^{e}\right), \\
& \mathbf{D}_{x}=\mathcal{A}_{x}\left(\mathbf{d}_{x}^{e}\right), \quad \mathbf{D}_{y}=\mathcal{A}_{y}\left(\mathbf{d}_{y}^{e}\right), \quad \mathbf{D}_{z}=\mathcal{A}_{z}\left(\mathbf{d}_{z}^{e}\right),
\end{aligned}
$$

tal que as submatrizes $\mathbf{m}_{\rho}^{e}, \mathbf{k}_{x x}^{e}, \mathbf{k}_{x y}^{e}, \mathbf{k}_{x z}^{e}, \mathbf{k}_{y x}^{e}, \mathbf{k}_{y y}^{e}, \mathbf{k}_{y z}^{e}, \mathbf{k}_{z x}^{e}, \mathbf{k}_{z y}^{e}, \mathbf{k}_{z z}^{e}, \mathbf{g}_{x}^{e}, \mathbf{g}_{y}^{e}, \mathbf{g}_{z}^{e}, \mathbf{d}_{x}^{e}, \mathbf{d}_{y}^{e}, \mathbf{d}_{z}^{e}$ que são matrizes definidas localmente para cada elemento, são dadas por

$$
\begin{gathered}
\mathbf{m}_{\rho, i j}^{e}=\int_{\Omega^{e}} \rho^{e} \psi_{i}^{e} \psi_{j}^{e} d \Omega \\
\mathbf{k}_{x x, i j}^{e}=\int_{\Omega^{e}} \mu^{e}\left(\frac{\partial \psi_{i}^{e}}{\partial x} \frac{\partial \psi_{j}^{e}}{\partial x}\right) d \Omega
\end{gathered}
$$




$$
\begin{gathered}
\mathbf{k}_{x y, i j}^{e}=\int_{\Omega^{e}} \mu^{e}\left(\frac{\partial \psi_{i}^{e}}{\partial y} \frac{\partial \psi_{j}^{e}}{\partial x}\right) d \Omega \\
\mathbf{k}_{x z, i j}^{e}=\int_{\Omega^{e}} \mu^{e}\left(\frac{\partial \psi_{i}^{e}}{\partial z} \frac{\partial \psi_{j}^{e}}{\partial x}\right) d \Omega \\
\mathbf{k}_{y x, i j}^{e}=\int_{\Omega^{e}} \mu^{e}\left(\frac{\partial \psi_{i}^{e}}{\partial x} \frac{\partial \psi_{j}^{e}}{\partial y}\right) d \Omega \\
\mathbf{k}_{y y, i j}^{e}=\int_{\Omega^{e}} \mu^{e}\left(\frac{\partial \psi_{i}^{e}}{\partial y} \frac{\partial \psi_{j}^{e}}{\partial y}\right) d \Omega \\
\mathbf{k}_{y z, i j}^{e}=\int_{\Omega^{e}} \mu^{e}\left(\frac{\partial \psi_{i}^{e}}{\partial z} \frac{\partial \psi_{j}^{e}}{\partial y}\right) d \Omega \\
\mathbf{k}_{z x, i j}^{e}=\int_{\Omega^{e}} \mu^{e}\left(\frac{\partial \psi_{i}^{e}}{\partial x} \frac{\partial \psi_{j}^{e}}{\partial z}\right) d \Omega \\
\mathbf{k}_{z y, i j}^{e}=\int_{\Omega^{e}} \mu^{e}\left(\frac{\partial \psi_{i}^{e}}{\partial y} \frac{\partial \psi_{j}^{e}}{\partial z}\right) d \Omega \\
\mathbf{d}_{y, k j}^{e}=\int_{\Omega^{e}} \mu^{e} \frac{\partial \psi_{i}^{e}}{\partial z} P_{k}^{e} d \Omega \\
\mathbf{d}_{z, i k}^{e}=\int_{\Omega^{e}} \mu^{e} \frac{\partial \psi_{i}^{e}}{\partial z} P_{k}^{e} d \Omega \\
\mathbf{k}_{z z, i j}^{e}=\int_{\Omega^{e}} \mu^{e}\left(\frac{\partial \psi_{i}^{e}}{\partial z} \frac{\partial \psi_{j}^{e}}{\partial z}\right) d \Omega \\
\mathbf{g}_{x, i k}^{e}=\int_{\Omega^{e}} \mu^{e} \frac{\partial \psi_{i}^{e}}{\partial x} P_{k}^{e} \frac{\partial \psi_{i}^{e}}{\partial y} P_{k}^{e} d \Omega \\
\mathbf{x}_{k}^{e} d \Omega
\end{gathered}
$$

O operador $\mathcal{A}$ que aparece acima é um operador que monta as submatrizes de elemento nas matrizes do sistema de EDOs 4.71, respeitando a correspondência entre os índices globais e locais dados nas equações 4.66), 4.67) e 4.68) .

As dimensões das matrizes que aparecem no sistema 4.71 são $N V \times N P$ para $\mathbf{G}_{x}, \mathbf{G}_{y}$ e $\mathbf{G}_{z}, N P \times N V$ para $\mathbf{D}_{x}, \mathbf{D}_{y}$ e $\mathbf{D}_{z}$ e $N V \times N V$ para todas as outras. A partir de (4.71) 
é possível escrever o sistema de EDOs de forma mais compacta, acoplando as velocidades nas direções $x, y$, e $z$ o que resulta

$$
\begin{gathered}
\mathbf{M}_{\rho} \frac{D u}{D t}-\frac{1}{R e} \mathbf{K} \mathbf{u}-\mathbf{G} \mathbf{p}-\frac{1}{F r^{2}} \mathbf{M}_{\rho} \mathbf{g}=0 \\
\mathbf{D u}=0
\end{gathered}
$$

onde agora as variáveis são definidas como $\mathbf{u}=\left[u_{1}, \ldots, u_{N V}, v_{1}, \ldots, v_{N V}, w_{1}, \ldots, w_{N V}\right]^{T}$, $\mathbf{g}=\left[g_{1}^{x}, \ldots, g_{N V}^{x}, g_{1}^{y}, \ldots, g_{N V}^{y}, g_{1}^{w}, \ldots, g_{N V}^{w}\right]^{T}$ e $\mathbf{p}=\left[p_{1}, \ldots, p_{N P}\right]^{T}$, e as matrizes dadas por

$$
\begin{gathered}
\mathbf{M}_{\rho}=\left[\begin{array}{ccc}
\mathbf{M}_{\rho, x} & \mathbf{0} & \mathbf{0} \\
\mathbf{0} & \mathbf{M}_{\rho, y} & \mathbf{0} \\
\mathbf{0} & \mathbf{0} & \mathbf{M}_{\rho, z}
\end{array}\right]_{3 N V \times 3 N V} \\
\mathbf{K}=\left[\begin{array}{lll}
\mathbf{K}_{x x} & \mathbf{K}_{x y} & \mathbf{K}_{x z} \\
\mathbf{K}_{y x} & \mathbf{K}_{y y} & \mathbf{K}_{y z} \\
\mathbf{K}_{z x} & \mathbf{K}_{z y} & \mathbf{K}_{z z}
\end{array}\right]_{3 N V \times 3 N V} \\
\mathbf{G}=\left[\begin{array}{l}
\mathbf{G}_{x} \\
\mathbf{G}_{y} \\
\mathbf{G}_{z}
\end{array}\right]_{3 N V \times N P} \quad \mathbf{D}=\left[\begin{array}{lll}
\mathbf{D}_{x} & \mathbf{D}_{y} & \mathbf{D}_{z}
\end{array}\right]_{N P \times 3 N V}
\end{gathered}
$$

\subsubsection{Cálculo das Matrizes nos Elementos}

Uma vez definidas as funções de interpolação, as matrizes definidas nas equações (4.72) a (5.5) podem ser calculadas para se obter os coeficientes das matrizes nos elementos, que contribuem na montagem da matriz global do sistema. Esta matriz global necessita do cálculo das matrizes individuais de cada elemento. Este cálculo é facilitado mapeandose os elementos do domínio real em um elemento de referência denominado de elemento mestre $\widehat{\Omega}$. Este mapeamento é feito em função das coordenadas globais dos nós do elemento e das funções de interpolação definidas em coordenadas locais no elemento. Pode-se dizer então que existe uma "transformação inversa", tal que pode-se escrever cada elemento $\Omega_{e}$ de coordenadas $(x, y, z)$ para um elemento que o represente em coordenadas $(\xi, \eta, \zeta)$. Assim, pode-se introduzir um mapeamento $T_{e}$ de $\widehat{\Omega}$ para $\Omega_{e}$, definido pela mudança de coordenadas 


$$
T_{e}:\left\{\begin{aligned}
x & =x(\xi, \eta, \zeta) \\
y & =y(\xi, \eta, \zeta) \\
z & =z(\xi, \eta, \zeta)
\end{aligned}\right.
$$

O elemento $\Omega_{e}$ pode ser visto como uma imagem de $\widehat{\Omega}$ sob o mapeamento $T_{e}$ definido por (4.90). Desta forma, a geração de uma malha completa de elementos finitos contendo $E$ elementos é vista como uma sequência de transformações $\left\{T_{1}, T_{2}, \ldots, T_{E}\right\}$ da forma (4.90), na qual cada elemento $\Omega_{e}$ é a imagem do elemento mestre $\widehat{\Omega}$ fixado sob um mapeamento de coordenadas $T_{e}$. Este processo é ilustrado simbolicamente na Figura 4.4 .

Supondo que as funções $x, y$ e $z$ são contínuas e diferenciáveis com respeito a $\xi, \eta$ e $\zeta$, então as infinitésimas $d \xi, d \eta$ e $d \zeta$ transformam-se em $d x, d y$ e $d z$ de acordo com

$$
\begin{aligned}
& d x=\frac{\partial x}{\partial \xi} d \xi+\frac{\partial x}{\partial \eta} d \eta+\frac{\partial x}{\partial \zeta} d \zeta \\
& d y=\frac{\partial y}{\partial \xi} d \xi+\frac{\partial y}{\partial \eta} d \eta+\frac{\partial y}{\partial \zeta} d \zeta \\
& d z=\frac{\partial z}{\partial \xi} d \xi+\frac{\partial z}{\partial \eta} d \eta+\frac{\partial z}{\partial \zeta} d \zeta
\end{aligned}
$$

as quais podem ser escritas na forma matricial

$$
\left[\begin{array}{l}
d x \\
d y \\
d z
\end{array}\right]=\left[\begin{array}{lll}
\frac{\partial x}{\partial \xi} & \frac{\partial x}{\partial \eta} & \frac{\partial x}{\partial \zeta} \\
\frac{\partial y}{\partial \xi} & \frac{\partial y}{\partial \eta} & \frac{\partial y}{\partial \zeta} \\
\frac{\partial z}{\partial \xi} & \frac{\partial y}{\partial \eta} & \frac{\partial z}{\partial \zeta}
\end{array}\right]\left[\begin{array}{l}
d \xi \\
d \eta \\
d \zeta
\end{array}\right]
$$

A matriz quadrada de derivadas parciais mostrada em (4.91) é chamada de matriz Jacobiana da transformação indicada em 4.90 e é denotada por J. Se, num ponto $(\xi, \eta, \zeta) \in \widehat{\Omega}$, é possível resolver o sistema (4.91) para $d \xi, d \eta$ e $d \zeta$ em termos de $d x, d y$ e $d z$, então um mapeamento inverso $T_{e}^{-1}$ das coordenadas $(x, y, z)$ para as coordenadas $(\xi, \eta, \zeta)$ pode ser construído neste ponto. A transformação de coordenadas é dada através do operador Jacobiano. Com ele é possível rotacionar e transladar o elemento para sua nova base $(\xi, \eta, \zeta)$ reduzindo ou aumentando seu volume original. Uma condição necessária e suficiente para o sistema (4.91) ser inversível é que o determinante $\mathbf{J}$ da matriz Jacobiana seja diferente 
de zero em $(\xi, \eta, \zeta) \in \widehat{\Omega}$.

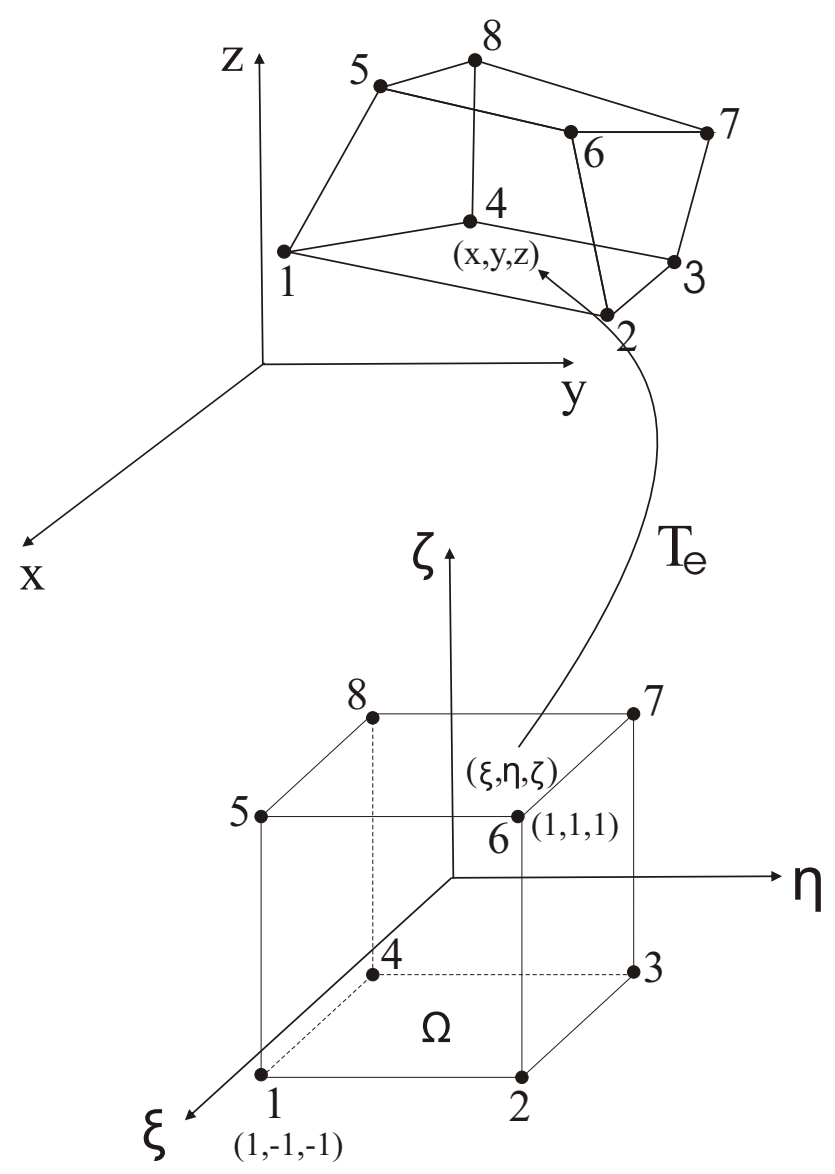

Figura 4.4: Elemento mestre cubo $\widehat{\Omega}$.

A função $|\mathbf{J}|$ é chamada de Jacobiana da transformação 4.90 e é dada por

$|\mathbf{J}|=\operatorname{det} \mathbf{J}=\frac{\partial x}{\partial \xi} \frac{\partial y}{\partial \eta} \frac{\partial z}{\partial \zeta}+\frac{\partial x}{\partial \eta} \frac{\partial y}{\partial \zeta} \frac{\partial z}{\partial \xi}+\frac{\partial x}{\partial \zeta} \frac{\partial y}{\partial \xi} \frac{\partial z}{\partial \eta}-\frac{\partial z}{\partial \xi} \frac{\partial y}{\partial \eta} \frac{\partial x}{\partial \zeta}-\frac{\partial z}{\partial \eta} \frac{\partial y}{\partial \zeta} \frac{\partial x}{\partial \xi}-\frac{\partial z}{\partial \zeta} \frac{\partial y}{\partial \xi} \frac{\partial x}{\partial \eta}$

Então, sempre que $|\mathbf{J}| \neq 0$, pode-se escrever

$$
\left[\begin{array}{l}
d \xi \\
d \eta \\
d \zeta
\end{array}\right]=\mathbf{J}^{-1}\left[\begin{array}{l}
d x \\
d y \\
d z
\end{array}\right]=
$$




$$
=\frac{1}{|\mathbf{J}|}\left[\begin{array}{ccc}
\frac{\partial y}{\partial \eta} \frac{\partial z}{\partial \zeta}-\frac{\partial z}{\partial \eta} \frac{\partial y}{\partial \zeta} & -\frac{\partial x}{\partial \eta} \frac{\partial z}{\partial \zeta}+\frac{\partial z}{\partial \eta} \frac{\partial x}{\partial \zeta} & \frac{\partial x}{\partial \eta} \frac{\partial y}{\partial \zeta}-\frac{\partial y}{\partial \eta} \frac{\partial x}{\partial \zeta} \\
-\frac{\partial y}{\partial \xi} \frac{\partial z}{\partial \zeta}+\frac{\partial z}{\partial \xi} \frac{\partial y}{\partial \zeta} & \frac{\partial x}{\partial \xi} \frac{\partial z}{\partial \zeta}-\frac{\partial z}{\partial \xi} \frac{\partial x}{\partial \zeta} & -\frac{\partial x}{\partial \xi} \frac{\partial y}{\partial \zeta}+\frac{\partial y}{\partial \xi} \frac{\partial x}{\partial \zeta} \\
\frac{\partial y}{\partial \xi} \frac{\partial z}{\partial \eta}-\frac{\partial z}{\partial \xi} \frac{\partial y}{\partial \eta} & -\frac{\partial x}{\partial \xi} \frac{\partial z}{\partial \eta}+\frac{\partial z}{\partial \xi} \frac{\partial x}{\partial \eta} & \frac{\partial x}{\partial \xi} \frac{\partial y}{\partial \eta}-\frac{\partial y}{\partial \xi} \frac{\partial x}{\partial \eta}
\end{array}\right]\left[\begin{array}{c}
d x \\
d y \\
d z
\end{array}\right]
$$

e

$$
T_{e}^{-1}:\left\{\begin{array}{l}
\xi=\xi(x, y, z) \\
\eta=\eta(x, y, z) \\
\zeta=\zeta(x, y, z)
\end{array}\right.
$$

definem o mapeamento do elemento $\Omega_{e}$ de volta para o elemento mestre $\widehat{\Omega}$. Note que da mesma forma que em 4.91,

$$
\left[\begin{array}{c}
d \xi \\
d \eta \\
d \zeta
\end{array}\right]=\left[\begin{array}{lll}
\frac{\partial \xi}{\partial x} & \frac{\partial \xi}{\partial y} & \frac{\partial \xi}{\partial z} \\
\frac{\partial \eta}{\partial x} & \frac{\partial \eta}{\partial y} & \frac{\partial \eta}{\partial z} \\
\frac{\partial \zeta}{\partial x} & \frac{\partial \zeta}{\partial y} & \frac{\partial \zeta}{\partial z}
\end{array}\right]\left[\begin{array}{l}
d x \\
d y \\
d z
\end{array}\right]
$$

Igualando os termos de (4.94) e (4.92), tem-se

$$
\begin{gathered}
\frac{\partial \xi}{\partial x}=\frac{1}{|\mathbf{J}|}\left(\frac{\partial y}{\partial \eta} \frac{\partial z}{\partial \zeta}-\frac{\partial z}{\partial \eta} \frac{\partial y}{\partial \zeta}\right), \\
\frac{\partial \xi}{\partial y}=\frac{1}{|\mathbf{J}|}\left(-\frac{\partial x}{\partial \eta} \frac{\partial z}{\partial \zeta}+\frac{\partial z}{\partial \eta} \frac{\partial x}{\partial \zeta}\right) \\
\frac{\partial \xi}{\partial z}=\frac{1}{|\mathbf{J}|}\left(\frac{\partial x}{\partial \eta} \frac{\partial y}{\partial \zeta}-\frac{\partial y}{\partial \eta} \frac{\partial x}{\partial \zeta}\right) \\
\frac{\partial \eta}{\partial x}=\frac{1}{|\mathbf{J}|}\left(-\frac{\partial y}{\partial \xi} \frac{\partial z}{\partial \zeta}+\frac{\partial z}{\partial \xi} \frac{\partial y}{\partial \zeta}\right), \\
\frac{\partial \eta}{\partial y}=\frac{1}{|\mathbf{J}|}\left(\frac{\partial x}{\partial \xi} \frac{\partial z}{\partial \zeta}-\frac{\partial z}{\partial \xi} \frac{\partial x}{\partial \zeta}\right) \\
\frac{\partial \eta}{\partial z}=\frac{1}{|\mathbf{J}|}\left(-\frac{\partial x}{\partial \xi} \frac{\partial y}{\partial \zeta}+\frac{\partial y}{\partial \xi} \frac{\partial x}{\partial \zeta}\right)
\end{gathered}
$$




$$
\begin{aligned}
\frac{\partial \zeta}{\partial x} & =\frac{1}{|\mathbf{J}|}\left(\frac{\partial y}{\partial \xi} \frac{\partial z}{\partial \eta}-\frac{\partial z}{\partial \xi} \frac{\partial y}{\partial \eta}\right) \\
\frac{\partial \zeta}{\partial y} & =\frac{1}{|\mathbf{J}|}\left(-\frac{\partial x}{\partial \xi} \frac{\partial z}{\partial \eta}+\frac{\partial z}{\partial \xi} \frac{\partial x}{\partial \eta}\right) \\
\frac{\partial \zeta}{\partial z} & =\frac{1}{|\mathbf{J}|}\left(\frac{\partial x}{\partial \xi} \frac{\partial y}{\partial \eta}-\frac{\partial y}{\partial \xi} \frac{\partial x}{\partial \eta}\right)
\end{aligned}
$$

Estas relações são cruciais nos cálculos de transformação em $\widehat{\Omega}$ para os cálculos correspondentes em cada elemento.

Existem várias maneiras de construir mapeamentos $T_{e}$ que satisfaçam os critérios descritos até então. Uma maneira de se construir o mapeamento é a utilização de funções interpoladoras. Suponha que as funções de forma $\psi_{j}$ estão definidas em um elemento mestre $\widehat{\Omega}$. Estas funções de forma podem ser usadas para construir uma aproximação $\widehat{g}$ de qualquer função $g=g(\xi, \eta, \zeta)$ por interpolação de $g$ nos pontos nodais,

$$
\widehat{g}(\xi, \eta, \zeta)=\sum_{j=1}^{M} g_{j} \widehat{\psi}_{j}(\xi, \eta, \zeta)
$$

onde $g_{j}=g\left(\xi_{j}, \eta_{j}, \zeta_{j}\right)$, e $\left(\xi_{j}, \eta_{j}, \zeta_{j}\right)$ como sendo as coordenadas do nó $j$, e a notação $\widehat{\psi}_{j}$ enfatiza que as funções de forma pertencem ao elemento mestre, e $M$ é o número de nós definidos no elemento mestre.

Considerando 4.96 e 4.90, nota-se que as variáveis de coordenadas $x, y$ e $z$ podem ser vistas como funções lineares em $\Omega_{e}$. Portanto as funções de forma podem ser utilizadas para construir o mapeamento $T_{e}$,

$$
\begin{aligned}
& x=\sum_{j=1}^{M} x_{j} \widehat{\psi}_{j}(\xi, \eta, \zeta) \\
& y=\sum_{j=1}^{M} y_{j} \widehat{\psi}_{j}(\xi, \eta, \zeta) \\
& z=\sum_{j=1}^{M} z_{j} \widehat{\psi}_{j}(\xi, \eta, \zeta)
\end{aligned}
$$

onde $x_{j}, y_{j}$ e $z_{j}$ são as coordenadas $x, y, z$ do ponto local nodal no elemento $\Omega_{e}$. 


\section{Transformações das Funções de Forma}

Considere $g$ uma função escalar de $(x, y, z)$ definida sobre um elemento $\Omega_{e}$. Pode-se converter $g$ para uma função $\widehat{g}$ de $(\xi, \eta, \zeta)$ definida em $\widehat{\Omega}$ fazendo

$$
g(x, y, z)=g(x(\xi, \eta, \zeta), y(\xi, \eta, \zeta), z(\xi, \eta, \zeta))=\widehat{g}(\xi, \eta, \zeta)
$$

onde $x(\xi, \eta, \zeta), y(\xi, \eta, \zeta)$ e $z(\xi, \eta, \zeta)$ são dados por (4.97), 4.98) e (4.99). Similarmente, se $\widehat{g}(\xi, \eta, \zeta)$ é dado sobre $\widehat{\Omega}$, então através da transformação de $(\xi, \eta, \zeta)$ em $(x, y, z)$,

$$
\widehat{g}(\xi, \eta, \zeta)=\widehat{g}(\xi(x, y, z), \eta(x, y, z), \zeta(x, y, z))=g(x, y, z)
$$

Portanto, o elemento da função de forma $\psi_{j}^{e}=\psi_{j}^{e}(x, y, z)$ para $\Omega_{e}$ são simplesmente obtidas de $\widehat{\psi}_{j}(\xi, \eta, \zeta)$ por

$$
\psi_{j}^{e}(x, y, z)=\widehat{\psi}_{j}(\xi(x, y, z), \eta(x, y, z), \zeta(x, y, z)), j=1,2, \ldots, M
$$

As derivadas de $\psi_{j}^{e}$ são obtidas pela regra da cadeia

$$
\begin{aligned}
& \frac{\partial \psi_{j}^{e}}{\partial x}(x, y, z)=\frac{\partial \psi_{j}}{\partial \xi} \frac{\partial \xi}{\partial x}+\frac{\partial \psi_{j}}{\partial \eta} \frac{\partial \eta}{\partial x}+\frac{\partial \psi_{j}}{\partial \zeta} \frac{\partial \zeta}{\partial x} \\
& \frac{\partial \psi_{j}^{e}}{\partial y}(x, y, z)=\frac{\partial \psi_{j}}{\partial \xi} \frac{\partial \xi}{\partial y}+\frac{\partial \psi_{j}}{\partial \eta} \frac{\partial \eta}{\partial y}+\frac{\partial \psi_{j}}{\partial \zeta} \frac{\partial \zeta}{\partial y} \\
& \frac{\partial \psi_{j}^{e}}{\partial z}(x, y, z)=\frac{\partial \psi_{j}}{\partial \xi} \frac{\partial \xi}{\partial z}+\frac{\partial \psi_{j}}{\partial \eta} \frac{\partial \eta}{\partial z}+\frac{\partial \psi_{j}}{\partial \zeta} \frac{\partial \zeta}{\partial z}
\end{aligned}
$$

De acordo com as equações 4.97, 4.98) e 4.99),

$$
\begin{gathered}
\frac{\partial x}{\partial \xi}=\sum_{k=1}^{M} x_{k} \frac{\partial \widehat{\psi}_{k}(\xi, \eta, \zeta)}{\partial \xi}, \quad \frac{\partial x}{\partial \eta}=\sum_{k=1}^{M} x_{k} \frac{\partial \widehat{\psi}_{k}(\xi, \eta, \zeta)}{\partial \eta}, \quad \frac{\partial x}{\partial \zeta}=\sum_{k=1}^{M} x_{k} \frac{\partial \widehat{\psi}_{k}(\xi, \eta, \zeta)}{\partial \zeta} \\
\frac{\partial y}{\partial \xi}=\sum_{k=1}^{M} y_{k} \frac{\partial \widehat{\psi}_{k}(\xi, \eta, \zeta)}{\partial \xi}, \quad \frac{\partial y}{\partial \eta}=\sum_{k=1}^{M} y_{k} \frac{\partial \widehat{\psi}_{k}(\xi, \eta, \zeta)}{\partial \eta}, \quad \frac{\partial y}{\partial \zeta}=\sum_{k=1}^{M} y_{k} \frac{\partial \widehat{\psi}_{k}(\xi, \eta, \zeta)}{\partial \zeta} \\
\frac{\partial z}{\partial \xi}=\sum_{k=1}^{M} z_{k} \frac{\partial \widehat{\psi}_{k}(\xi, \eta, \zeta)}{\partial \xi}, \quad \frac{\partial z}{\partial \eta}=\sum_{k=1}^{M} z_{k} \frac{\partial \widehat{\psi}_{k}(\xi, \eta, \zeta)}{\partial \eta}, \quad \frac{\partial z}{\partial \zeta}=\sum_{k=1}^{M} z_{k} \frac{\partial \widehat{\psi}_{k}(\xi, \eta, \zeta)}{\partial \zeta}
\end{gathered}
$$


Portanto, usando 4.95 e 4.104, as equações 4.103 se tornam

$$
\begin{aligned}
& \frac{\partial \psi_{j}}{\partial x}(x, y, z)=\frac{1}{|J(\xi, \eta, \zeta)|}\left\{\frac{\partial \psi_{j}}{\partial \xi}\left(\sum_{k=1}^{M} y_{k} \frac{\partial \psi_{k}}{\partial \eta} \sum_{k=1}^{M} z_{k} \frac{\partial \psi_{k}}{\partial \zeta}-\sum_{k=1}^{M} z_{k} \frac{\partial \psi_{k}}{\partial \eta} \sum_{k=1}^{M} y_{k} \frac{\partial \psi_{k}}{\partial \zeta}\right)+\right. \\
& +\frac{\partial \psi_{j}}{\partial \eta}\left(-\sum_{k=1}^{M} y_{k} \frac{\partial \psi_{k}}{\partial \xi} \sum_{k=1}^{M} z_{k} \frac{\partial \psi_{k}}{\partial \zeta}+\sum_{k=1}^{M} z_{k} \frac{\partial \psi_{k}}{\partial \xi} \sum_{k=1}^{M} y_{k} \frac{\partial \psi_{k}}{\partial \zeta}\right)+ \\
& \left.+\frac{\partial \psi_{j}}{\partial \zeta}\left(\sum_{k=1}^{M} y_{k} \frac{\partial \psi_{k}}{\partial \xi} \sum_{k=1}^{M} z_{k} \frac{\partial \psi_{k}}{\partial \eta}-\sum_{k=1}^{M} z_{k} \frac{\partial \psi_{k}}{\partial \xi} \sum_{k=1}^{M} y_{k} \frac{\partial \psi_{k}}{\partial \eta}\right)\right\} \\
& \frac{\partial \psi_{j}}{\partial y}(x, y, z)=\frac{1}{|J(\xi, \eta, \zeta)|}\left\{\frac{\partial \psi_{j}}{\partial \xi}\left(-\sum_{k=1}^{M} x_{k} \frac{\partial \psi_{k}}{\partial \eta} \sum_{k=1}^{M} z_{k} \frac{\partial \psi_{k}}{\partial \zeta}+\sum_{k=1}^{M} z_{k} \frac{\partial \psi_{k}}{\partial \eta} \sum_{k=1}^{M} x_{k} \frac{\partial \psi_{k}}{\partial \zeta}\right)+\right. \\
& +\frac{\partial \psi_{j}}{\partial \eta}\left(\sum_{k=1}^{M} x_{k} \frac{\partial \psi_{k}}{\partial \xi} \sum_{k=1}^{M} z_{k} \frac{\partial \psi_{k}}{\partial \zeta}-\sum_{k=1}^{M} z_{k} \frac{\partial \psi_{k}}{\partial \xi} \sum_{k=1}^{M} x_{k} \frac{\partial \psi_{k}}{\partial \zeta}\right)+ \\
& \left.+\frac{\partial \psi_{j}}{\partial \zeta}\left(-\sum_{k=1}^{M} x_{k} \frac{\partial \psi_{k}}{\partial \xi} \sum_{k=1}^{M} z_{k} \frac{\partial \psi_{k}}{\partial \eta}+\sum_{k=1}^{M} z_{k} \frac{\partial \psi_{k}}{\partial \xi} \sum_{k=1}^{M} x_{k} \frac{\partial \psi_{k}}{\partial \eta}\right)\right\} \\
& \frac{\partial \psi_{j}}{\partial z}(x, y, z)=\frac{1}{|J(\xi, \eta, \zeta)|}\left\{\frac{\partial \psi_{j}}{\partial \xi}\left(\sum_{k=1}^{M} x_{k} \frac{\partial \psi_{k}}{\partial \eta} \sum_{k=1}^{M} y_{k} \frac{\partial \psi_{k}}{\partial \zeta}-\sum_{k=1}^{M} y_{k} \frac{\partial \psi_{k}}{\partial \eta} \sum_{k=1}^{M} x_{k} \frac{\partial \psi_{k}}{\partial \zeta}\right)+\right. \\
& +\frac{\partial \psi_{j}}{\partial \eta}\left(-\sum_{k=1}^{M} x_{k} \frac{\partial \psi_{k}}{\partial \xi} \sum_{k=1}^{M} y_{k} \frac{\partial \psi_{k}}{\partial \zeta}+\sum_{k=1}^{M} y_{k} \frac{\partial \psi_{k}}{\partial \xi} \sum_{k=1}^{M} x_{k} \frac{\partial \psi_{k}}{\partial \zeta}\right)+ \\
& \left.+\frac{\partial \psi_{j}}{\partial \zeta}\left(\sum_{k=1}^{M} x_{k} \frac{\partial \psi_{k}}{\partial \xi} \sum_{k=1}^{M} y_{k} \frac{\partial \psi_{k}}{\partial \eta}-\sum_{k=1}^{M} y_{k} \frac{\partial \psi_{k}}{\partial \xi} \sum_{k=1}^{M} x_{k} \frac{\partial \psi_{k}}{\partial \eta}\right)\right\}
\end{aligned}
$$

onde $|\mathbf{J}(\xi, \eta)|$ é a Jacobiana da transformação $T_{e}$. Note que as derivadas parciais de $\psi_{j}^{e}$ com respeito a $x, y$ e $z$ são completamente determinadas por cálculos definidos somente sobre o elemento mestre $\widehat{\Omega}$.

\subsubsection{Integração Numérica}

Após definidas as matrizes dos elementos, deve-se fazer o cáculo das integrais.

Integrais de funções sobre cada elemento $\Omega_{e}$ podem ser calculadas usando cálculos 
definidos somente sobre o elemento mestre $\widehat{\Omega}$ pela relação

$$
\int_{\Omega_{e}} g(x, y, z) d x d y d z=\int_{\widehat{\Omega}} \widehat{g}(\xi, \eta, \zeta)|\mathbf{J}(\xi, \eta, \zeta)| d \xi d \eta d \zeta
$$

Portanto, as integrais das funções sobre cada elemento $\Omega_{e}$ podem ser obtidas usando cálculos sobre $\widehat{\Omega}$ através desta simples relação.

Em cálculos reais, as fórmulas numéricas de quadratura são geralmente usadas para resolver todas as integrais. O método analítico de solução fornece valores exatos para as integrais, porém dependendo do tipo de elemento escolhido, suas funções de forma apresentam graus elevados e de difícil manipulação. Dependendo da quantidade de pontos de quadratura a integração numérica é exata e equivalente ao método analítico. Suas grandes vantagens se encontram na implementação do código, relativamente simples, e na facilidade de troca de elementos, pois a estrutura do código se mantém inalterada.

Em geral, qualquer fórmula de quadratura Gaussiana ou outra é definida especificando as coordenadas $\left(\xi_{l}, \eta_{l}, \zeta_{l}\right)$ de um número $N_{l}$ de pontos de integração no domínio sobre o qual a integral será resolvida, assim como um conjunto de $N_{l}$ números $w_{l}$ chamados pesos de quadratura $\left(l=1,2, \ldots, N_{l}\right)$. Portanto, se $\widehat{G}(\xi, \eta, \zeta)=\widehat{g}(\xi, \eta, \zeta)|\mathbf{J}(\xi, \eta, \zeta)|$ precisa ser integrado sobre $\widehat{\Omega}$, usa-se a fórmula

$$
\int_{\Omega_{e}} g(x, y, z) d x d y d z=\int_{\widehat{\Omega}} \widehat{G}(\xi, \eta, \zeta) d \xi d \eta d \zeta=\sum_{l=1}^{N_{l}} \widehat{G}\left(\xi_{l}, \eta_{l}, \zeta_{l}\right) w_{l}+\widehat{E}
$$

onde $\widehat{E}$ é o erro da quadratura.

\section{Integração Unidimensional}

Para o caso unidimensional, tem-se

$$
\int_{\widehat{\Omega}} G(\xi) d \xi=\int_{-1}^{1} G(\xi) d \xi
$$

que pode ser aproximada por

$$
\int_{\widehat{\Omega}} G(\xi) d \xi=\int_{-1}^{1} G(\xi) d \xi \approx \sum_{i=1}^{N} G\left(\xi_{i},\right) w_{i}
$$


onde $N$ é o número total de pontos de integração, $\xi_{i}$ são os pontos de integração e $w_{i}$ os respectivos pesos. A Tabela 4.1 mostra os pontos de integração e os pesos para uma reta.

\begin{tabular}{ccc}
\hline Numero de pontos & Pontos de quadratura $\left(\xi_{i}\right)$ & Pesos $\left(w_{i}\right)$ \\
\hline 1 & 0.000000000000000 & 2.0000000000000000 \\
2 & $\xi_{1}, \xi_{2}=0.577350269189626$ & 1.0000000000000000 \\
3 & $\xi_{1}, \xi_{3}=0.774596669241483$ & 0.5555555555555555 \\
& $\xi_{2}=0.000000000000000$ & 0.8888888888888889 \\
& & \\
4 & $\xi_{1}, \xi_{4}=0.861136311594053$ & 0.34785485147454 \\
& $\xi_{2}, \xi_{3}=0.339981043584856$ & 0.65145154862546 \\
\hline
\end{tabular}

Tabela 4.1: Coeficientes abscissa e peso da fórmula de quadratura gaussiana para uma reta.

\section{Integração de Elementos Bidimensionais}

Para casos bidimensionais em regiões retangulares, tem-se

$$
\begin{gathered}
\int_{\widehat{\Omega}} G(\xi, \eta) d \xi d \eta=\int_{-1}^{1} \int_{-1}^{1} G(\xi, \eta) d \xi d \eta=\int_{-1}^{1}\left[\sum_{i=1}^{N} G\left(\xi_{i}, \eta\right) w_{i}\right] d \eta= \\
\sum_{j=1}^{N} w_{j}\left[\sum_{i=1}^{N} G\left(\xi_{i}, \eta_{j}\right) w_{i}\right]=\sum_{j=1}^{N} \sum_{i=1}^{N} G\left(\xi_{i}, \eta_{j}\right) w_{i} w_{j}
\end{gathered}
$$

As integrais envolvidas para elementos triangulares podem ser definidas em termos de coordenada de área

$$
\int_{\widehat{\Omega}} G\left(L_{1}, L_{2}, L_{3}\right) d L_{2} d L_{3}, \quad\left(L_{1}=1-L_{2}-L_{3}\right)
$$

que pode ser aproximada por

$$
\int_{\widehat{\Omega}} G\left(L_{1}, L_{2}, L_{3}\right) d L_{2} d L_{3} \approx \sum_{i=1}^{N} w_{i} G\left(L_{1}^{(i)}, L_{2}^{(i)}, L_{3}^{(i)}\right)
$$

A Tabela 4.2 mostra alguns pontos de quadratura e pesos para elementos triangulares. 


\begin{tabular}{ccc}
\hline Ordem & Coordenadas triangulares $\left(L_{1}, L_{2}, L_{3}\right)$ & Pesos $\left(w_{i}\right)$ \\
\hline linear & $\frac{1}{3}, \frac{1}{3}, \frac{1}{3}$ & 1 \\
\hline \multirow{2}{*}{ quadrático (1) } & $\frac{1}{2}, \frac{1}{2}, 0$ & $\frac{1}{3}$ \\
& $0, \frac{1}{2}, \frac{1}{2}$ & $\frac{1}{3}$ \\
$\frac{1}{2}, 0, \frac{1}{2}$ & $\frac{1}{3}$ \\
\hline \multirow{2}{*}{ quadrático (2) } & $\frac{1}{6}, \frac{1}{6}, \frac{4}{6}$ & $\frac{1}{3}$ \\
& $\frac{2}{3}, \frac{1}{6}, \frac{1}{6}$ & $\frac{1}{3}$ \\
& $\frac{1}{6}, \frac{2}{3}, \frac{1}{6}$ & $\frac{1}{3}$ \\
\hline \multirow{2}{*}{ cúbico } & $\frac{1}{3}, \frac{1}{3}, \frac{1}{3}$ & \\
& $0.6,0.2,0.2$ & $-\frac{27}{48}$ \\
& $0.2,0.6,0.2$ & $\frac{25}{48}$ \\
& $0.2,0.2,0.6$ & $\frac{25}{48}$ \\
& & $\frac{25}{48}$ \\
\hline
\end{tabular}

Tabela 4.2: Coordenadas e pesos da fórmula de quadratura gaussiana para elementos triangulares.

\section{Integração para Elementos Prismáticos}

Para integração em regiões prismáticas, onde o domínio do elemento mestre está definido em $\xi \in[0,1], \eta \in[0,1]$ e $\zeta \in[-1,1]$, pode-se resolver a integral da seguinte forma

$$
\int_{\widehat{\Omega}} G(\xi, \eta, \zeta) d \xi d \eta d \zeta=\int_{-1}^{1} \int_{0}^{1} \int_{0}^{1} G(\xi, \eta, \zeta) d \xi d \eta d \zeta=\sum_{i=1}^{N} \sum_{j=1}^{N} \sum_{k=1}^{N} w_{i} w_{j} w_{k} G\left(\xi_{i}, \eta_{j}, \zeta_{k}\right)
$$

onde $N$ é o número de pontos de integração em cada direção.

Neste trabalho foram utilizados elementos prismáticos lineares. Para tanto, os pontos de quadratura e os pesos para elementos prismáticos lineares são obtidos a partir da combinação dos pontos e pesos de um elemento triangular quadrático com os de um elemento linear de ordem $n=2$. Os pontos de quadratura são então reunidos e os pesos correspondentes são multiplicados. A Tabela 4.3 mostra os pontos de quadratura e os respectivos pesos utilizados na integração de um elemento prismático linear. 


\begin{tabular}{ccccc}
\hline$n o ́$ & $\xi_{i}$ & $\left(\eta_{j}\right)$ & $\left(\zeta_{k}\right)$ & Pesos $\left(w_{i, j, k}\right)$ \\
\hline 1 & 0.166666666667 & 0.166666666667 & -0.577350269190 & $\frac{1}{3}$ \\
\hline 2 & 0.166666666667 & 0.6666666666667 & -0.577350269190 & $\frac{1}{3}$ \\
\hline 3 & 0.666666666667 & 0.166666666667 & -0.577350269190 & $\frac{1}{3}$ \\
\hline 4 & 0.166666666667 & 0.166666666667 & 0.577350269190 & $\frac{1}{3}$ \\
\hline 5 & 0.166666666667 & 0.666666666667 & 0.577350269190 & $\frac{1}{3}$ \\
\hline 6 & 0.666666666667 & 0.1666666666667 & 0.577350269190 & $\frac{1}{3}$ \\
\hline
\end{tabular}

Tabela 4.3: Pontos de quadratura e pesos utilizados na integração para elementos prismáticos lineares.

\subsection{Discretização Temporal Pelo Método Semi- Lagrangeano}

Depois de discretizar no espaço as equações de Navier-Stokes utilizando o método de Galerkin, obteve-se um conjunto de equações diferenciais ordinárias de primeira ordem, cujas derivadas podem ser discretizadas no tempo. O objetivo desta seção é apresentar a formulação semi-lagrangeana para a discretização temporal dos termos convectivos das equações.

O método semi-lagrangeano tem se mostrado consistente para as equações de NavierStokes. A derivada material de um escalar $u$ é dada no espaço tridimensional por

$$
\frac{D u}{D t}=\frac{\partial u}{\partial t}+u \frac{\partial u}{\partial x}+v \frac{\partial u}{\partial y}+w \frac{\partial u}{\partial z}
$$

A idéia básica do método semi-lagrangeano é acompanhar uma partícula de fluido durante sua trajetória ao longo da malha sobre o escoamento. A utilização do método é explícita, onde é necessária a informação dos valores da velocidade no tempo atual. Assim, o método aproxima estes valores referentes ao tempo anterior na trajetória. Basicamente a formulação semi-lagrangeana é dada por 


$$
\frac{D u}{D t}(p)=\frac{u_{p}^{n+1}-u_{p^{*}}^{n}}{\Delta t}
$$

e

$$
p^{*}=p-\Delta t \mathbf{u}_{p}
$$

onde $p$ é um ponto qualquer na malha e $p^{*}$ define o ponto $p$ no tempo anterior. O cálculo de $\mathbf{u}$ no ponto $p^{*}$ é feito por uma interpolação linear entre os pontos vizinhos. Esta interpolação é dependente de onde o ponto $p^{*}$ estiver localizado dentro do domínio como: sobre uma aresta, sobre um vértice, no interior de um elemento ou também fora do domínio.

\subsection{Método de Desacoplamento Baseado em Decom- posição LU}

Após a discretização espacial e temporal das equações o resultado final é um sistema de equações diferenciais. Para a resolução desse sistema existem vários métodos, mas pelo fato de apresentarem grande número de equações é importante escolher técnicas que diminuem os custos computacionais. Por esta razão, a escolha por um método desacoplado para a resolução do sistema tornou-se mais vantajosa.

O método utilizado para desacoplar as propriedades de velocidade e pressão é obtido através de uma fatoração em blocos do sistema linear resultante, onde o desacoplamento é feito após a discretização espacial das equações. Mais detalhes podem ser encontrados em Sousa, 2005.

Sejam as equações de Navier-Stokes discretizadas espacialmente pelo método de elementos finitos. Tais equações, discretizadas no tempo por um esquema semi-implícito, são dadas por

$$
\begin{gathered}
\mathbf{M} \rho\left(\frac{\mathbf{u}^{n+1}-\mathbf{u}_{*}^{n}}{\Delta t}\right)-\frac{1}{R e} \mathbf{K} \mathbf{u}^{n+1}-\mathbf{G}^{n+1}-\frac{1}{F r^{2}} \mathbf{M}_{\rho} \mathbf{g}=0 \\
\mathbf{D} \mathbf{u}^{n+1}=0
\end{gathered}
$$

Reescrevendo a equação (4.119) tem-se

$$
\left(\mathbf{M}_{\rho}-\frac{\Delta t}{R e} \mathbf{K}\right) \mathbf{u}^{n+1}=-\Delta t\left(\mathbf{G p}^{n+1}-\frac{1}{F r^{2}} \mathbf{M}_{\rho} \mathbf{g}\right)+\mathbf{M}_{\rho} \mathbf{u}_{*}^{n}
$$


que juntamente com 4.120 formam um sistema de equações que pode ser representado da seguinte forma

$$
\left[\begin{array}{cc}
\mathbf{B} & -\Delta t \mathbf{G} \\
\mathbf{D} & \mathbf{0}
\end{array}\right]\left[\begin{array}{l}
\mathbf{u}^{n+1} \\
\mathbf{p}^{n+1}
\end{array}\right]=\left[\begin{array}{c}
\mathbf{r}^{n} \\
\mathbf{0}
\end{array}\right]+\left[\begin{array}{l}
\mathbf{b c}_{\mathbf{1}} \\
\mathbf{b c}_{\mathbf{2}}
\end{array}\right]
$$

onde agora o sistema é escrito apenas para as incógnitas do problema, ou seja, $\mathbf{u}^{n+1}=\left[u_{1}^{n+1}, \ldots, u_{N u}^{n+1}, v_{1}^{n+1}, \ldots, v_{N v}^{n+1}, w_{1}^{n+1}, \ldots, w_{N w}^{n+1}\right]^{T}, p^{n+1}=\left[p_{1}^{n+1}, \ldots, p_{N p}^{n+1}\right]^{T}$, onde $N u, N v, N w$ e $N p$ são o número de incógnitas (nós livres) para a velocidade na direção $x$, velocidade na direção $y$, velocidade na direção $z$ e pressão respectivamente. A matriz $\mathbf{B}$ é dada por

$$
\mathbf{B}=\mathbf{M}_{\rho}-\frac{\Delta t}{R e} \mathbf{K}
$$

e o lado direito representa as grandezas conhecidas no tempo $n$, mais as condições de contorno que são as contribuições dos valores conhecidos de velocidade e pressão no lado direito do sistema

$$
\mathbf{r}^{n}=-\Delta t\left(-\frac{1}{F r^{2}} \mathbf{M}_{\rho} \mathbf{g}\right)+\mathbf{M}_{\rho} \mathbf{u}_{*}^{n}
$$

O método de desacoplamento consiste em decompor a matriz 4.122 através de uma fatoração por blocos. São apresentadas em Lee Lee et al., 2001 algumas formas de se fatorar exatamente esta matriz, onde cada forma dá origem a uma família de métodos diferentes. Utilizando uma fatoração canônica LU por blocos, obtém-se o seguinte sistema

$$
\left[\begin{array}{cc}
\mathbf{B} & \mathbf{0} \\
\mathbf{D} & \Delta t \mathbf{D B}^{-1} \mathbf{G}
\end{array}\right]\left[\begin{array}{cc}
\mathbf{I} & -\Delta t \mathbf{B}^{-1} \mathbf{G} \\
\mathbf{0} & \mathbf{I}
\end{array}\right]\left[\begin{array}{l}
\mathbf{u}^{n+1} \\
\mathbf{p}^{n+1}
\end{array}\right]=\left[\begin{array}{c}
\mathbf{r}^{n} \\
\mathbf{0}
\end{array}\right]+\left[\begin{array}{l}
\mathbf{b c}_{1} \\
\mathbf{b c}_{\mathbf{2}}
\end{array}\right]
$$

O sistema dado em 4.125, se resolvido, dá origem a um método conhecido como método de Uzawa Chang et al., 2002. No entanto, este método possui solução cara computacionalmente, pelo fato de inverter a matriz $\mathbf{B}$ a cada iteração. Métodos práticos para a solução de 4.125 aproximam a inversa de $\mathbf{B}$ pelas matrizes $\mathbf{B}_{\mathbf{1}}$ e $\mathbf{B}_{\mathbf{2}}$ de modo que o sistema pode ser escrito como

$$
\left[\begin{array}{cc}
\mathbf{B} & \mathbf{0} \\
\mathbf{D} & \Delta t \mathbf{D B}_{\mathbf{1}} \mathbf{G}
\end{array}\right]\left[\begin{array}{cc}
\mathbf{I} & -\Delta t \mathbf{B}_{2} \mathbf{G} \\
\mathbf{0} & \mathbf{I}
\end{array}\right]\left[\begin{array}{c}
\mathbf{u}^{n+1} \\
\mathbf{p}^{n+1}
\end{array}\right]=\left[\begin{array}{c}
\mathbf{r}^{n} \\
\mathbf{0}
\end{array}\right]+\left[\begin{array}{c}
\mathbf{b c}_{1} \\
\mathbf{b c}_{\mathbf{2}}
\end{array}\right]
$$


Por Chang Chang et al., 2002, podem ser feitas diferentes aproximações para as matrizes $\mathbf{B}_{1}$ e $\mathbf{B}_{2}$ de maneira a controlar o erro feito na separação das equações. Se $\mathbf{B}_{1}=\mathbf{B}_{2}$, então a equação da continuidade (4.120) é satisfeita, e todo erro das aproximações aparece na equação de conservação de quantidade de movimento. Para escoamentos incompressíveis é importante que a equação da continuidade seja satisfeita, para que haja conservação de massa no problema.

Pode-se fazer uma aproximação da inversa $\mathbf{B}^{-1}$ considerando $\mathbf{B}_{1}=\mathbf{B}_{2}=\mathbf{M}_{\rho}^{-1}$ Chang et al., 2002, e assim, o sistema 4.126) fica

$$
\begin{gathered}
\mathbf{B} \widetilde{\mathbf{u}}=\mathbf{r}^{n}+\mathbf{b} \mathbf{c}_{1} \\
\Delta t \mathbf{D} \mathbf{M}_{\rho}^{-1} \mathbf{G}^{n+1}=-\mathbf{D} \widetilde{\mathbf{u}}+\mathbf{b} \mathbf{c}_{2} \\
\mathbf{u}^{n+1}=\widetilde{\mathbf{u}}+\Delta t \mathbf{M}_{\rho}^{-1} \mathbf{G p}^{n+1}
\end{gathered}
$$

Um procedimento para a solução das equações é dado na seguinte ordem:

- Passo 1: Calcula-se $\widetilde{\mathbf{u}}$ de 4.127;

- Passo 2: Calcula-se $\mathbf{p}^{n+1}$ de 4.128);

- Passo 3: Encontra-se a velocidade final $\mathbf{u}^{n+1}$ usando 4.129);

\subsection{Considerações Finais}

Neste capítulo foram apresentadas as equações de Navier-Stokes escritas em sua formulação variacional. Uma breve discussão sobre o método de elementos finitos foi feita. O método de Galerkin foi apresentado para discretizar as equações de Navier-Stokes no espaço e o método semi-lagrangeano para discretizar os termos convectivos.

As matrizes utilizadas na solução das equações foram definidas para cada elemento, assim como a estratégia para o cálculo das integrais.

Foi apresentado o método utilizado para a resolução do sistema linear resultante possibilitando o desacoplamento das velocidades e pressão.

No próximo capítulo será apresentado o simulador GesarSim 3D e alguns detalhes da implementação das equações. 


\section{Capítulo \\ 5 \\ O Simulador GesarSim 3D}

Neste capítulo são apresentadas as estratégias utilizadas no desenvolvimento do simulador GesarSim 3D, responsável pela resolução das equações de Navier-Stokes para domínios tridimensionais de escoamento de fluido.

\subsection{Introdução}

A discretização por elementos finitos, quando aplicada às equações de Navier-Stokes, resulta em um sistema acoplado de equações não-lineares. O termo convectivo apresenta produto de incógnitas, caracterizando à não-linearidade do problema gerando operadores não simétricos de difícil solução tornando-se desta forma a escolha dos algoritmos de solução um assunto de suma importância, especialmente com respeito às propriedades de convergência. A busca por um método de solução ideal, pode muitas vezes ser uma tarefa difícil, dada a existência de diversas técnicas e suas variantes. No entanto, para a solução desse sistema optou-se por um método baseado em decomposição LU, o que possibilitou o desacoplamento de velocidade e pressão.

A existência de um módulo GesarSim 2D já consolidado auxiliou no cálculo da pressão no simulador GesarSim 3D, onde, através de uma aproximação hidrostática, a pressão foi atribuída a níveis inferiores no domínio. 


\subsection{Visão Geral do Sistema}

O sistema tem como objetivo a simulação numérica de escoamentos de fluidos newtonianos tridimensionais utilizando malhas estruturas e não estruturadas. Os usuários necessitam de resultados numéricos para, por exemplo, simular a injeção de moldes com geometria complexa. Estes resultados são as propriedades (velocidades, pressão) do fluido simulado, que podem ser visualizadas no final da simulação.

O sistema de simulação para malhas estruturadas e não estruturadas é um sistema computacional para modelagem, simulação e visualização de escoamentos de fluidos que possibilita a análise e a observação do comportamento dinâmico de fluidos incompressíveis.

Desta forma, esse sistema divide-se em três módulos:

- Gerador de Malha: este módulo é responsável pela geração da malha a ser utilizada na solução numérica das equações;

- Simulador: este módulo é responsável pela implementação das equações que regem o escoamento. Este módulo é dividido em:

- Hidrodinâmico: responsável pela resolução das equações governantes;

- Thermo: responsável pela resolução da equação da temperatura;

- Espécies: responsável pela resolução das equações de concentrações de espécies constituintes;

- Visualizador: este módulo é responsável pela visualização dos resultados obtidos pelo simulador.

Este trabalho foca o desenvolvimento do módulo Simulador Hidrodinâmico, especificamente para escoamentos de fluidos em domínios tridimensionais, fazendo uso de uma aproximação hidrostática para o cálculo da pressão. A seguir, será descrito como o domínio do problema é répresentado.

\subsection{Representação do Domínio}

O domínio tridimensional, composto por prismas lineares, foi representado utilizando-se uma estrutura de dados bidimensional chamada Opposite Face (OF), que representa a 
superfície do domínio. As múltiplas camadas triangulares foram representadas pelo espelhamento geométrico da superfície em cada nível criado, formando desta maneira prismas lineares. Este espelhamento pôde ser realizado pois tanto a base quanto o topo dos prisma utilizados são paralelos, onde o fator tridimensional foi conseguido através da definição de um vetor "profundidade", onde o número de posições determina quantas camadas de prismas constituem o domínio. Assim, quanto mais camadas de prismas existirem, maior será a divisão do volume em prismas, uma vez fixada a altura do domínio.

A Figura 5.1 ilustra uma parte do domínio, e define a notação aqui definida sobre níveis e camadas. Na figura, os níveis representam as posiçoes no vetor de profundidade, e cada dois níveis consecutivos formam uma camada de prismas, como pode ser observado. Note que cada camada pode ter uma altura diferente das outras, onde esta informação depende do valor prescrito no vetor de profundidade, referenciado aqui por "deep".

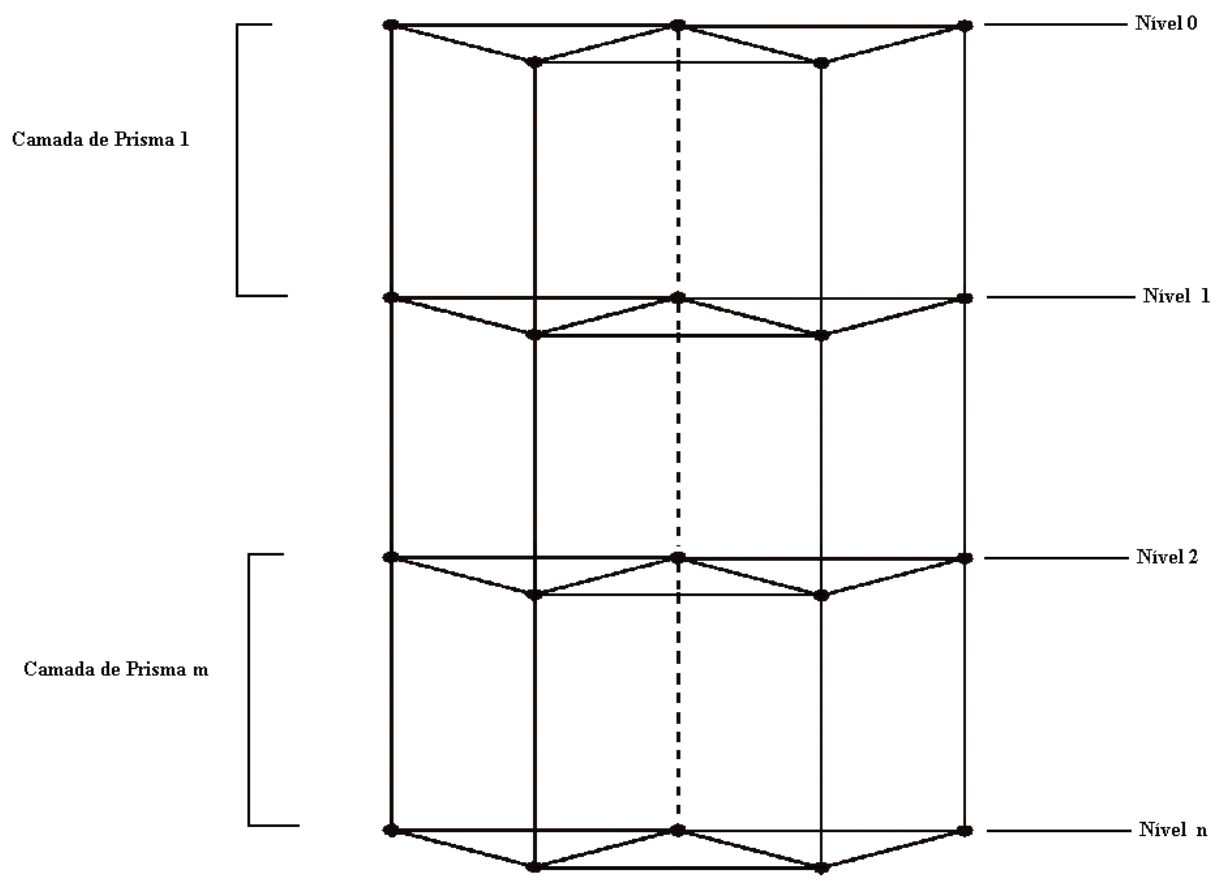

Figura 5.1: Esquema de representação do domínio.

Uma vez que a estrutura OF manterá informações da malha na superfície do domínio tridimensional, foi feito um controle da identificação dos vértices nos vários níveis existentes. Um esquema para geração de identificadores $(i d s)$ para níveis inferiores é fazer com que os vértices inferiores sejam múltiplos do número de vértices da superfície. Por exemplo, se tivermos uma malha superficial contendo dez vértices (ids de 0 a 9), os vértices 
inferiores são calculados da forma

$$
i d_{i}=i d_{0}+10 * \text { nivel }_{i}
$$

onde $i d_{i}$ é o novo $i d$ do vértice $i, i d_{0}$ é o $i d$ do vértice que possui as mesmas coordenadas $x, y$ de $i d_{i}$ mas está na superfície do domínio e contido na malha, e nivel $_{i}$ é o nível do vértice $i$ dentre as várias camadas.

É importante ressaltar que na superfície do domínio (tampa) são utilizados elementos do tipo "mini-elemento", pois oferecem uma maior estabilidade nos cálculos de velocidade, uma vez que este tipo de elemento é linear para a velocidade e pressão, e possui um grau de liberdade a mais no centróide do triângulo.

Este esquema de geração automática de identificadores é essencial para a associação de cada vértice do domínio nas matrizes globais definidas no processo de resolução numérica.

Um dos casos de simulação abordados neste trabalho envolve a inclusão de escadas em um canal retilínio. A representação das escadas no domínio foi feita tomando-se base um vetor de profundidades adicional. Este vetor associa, para cada elemento da superfície do domínio, a profundidade naquela direção ao fundo. Deste modo, dado um vértice qualquer é possível determinar se este localiza-se acima daquela profundidade (dentro do domínio), exatamente naquela profundidade (na escada / fundo do canal) ou embaixo da escada (vértice excluído do domínio).

Elementos formados por vértices excluídos do domínio também são excluídos, e suas contribuições locais nas matrizes globais do sistema são nulas, ou seja, para cada linha e coluna com índice referente a um vértice excluído, atribuem-se valores nulos às linhas e colunas da matriz global referentes àquele índice, e valor um à diagonal.

\subsection{Método Utilizado no Simulador GesarSim 3D}

O método numérico utilizado para simular escoamentos incompressíveis em malhas estruturadas e não estruturadas formadas por elementos prismáticos é baseado no método da decomposição LU, na formulação semi-lagrangeana para os termos convectivos e no método de elementos finitos.

Sejam as equações de conservação e continuidade dadas em sua forma adimensional

$$
\nabla \cdot \mathbf{u}=0
$$




$$
\frac{D(\rho \mathbf{u})}{D t}=-\nabla p+\frac{1}{R e} \nabla \cdot\left[\mu\left(\nabla \mathbf{u}+\nabla \mathbf{u}^{T}\right)\right]+\frac{1}{F r^{2}} \rho \mathbf{g},
$$

Substituindo o termo da derivada material pela formulação semi-lagrangeana dada por

$$
\frac{D(\rho \mathbf{u})}{D t}=\frac{u_{p}^{n+1}-u_{p_{*}}^{n}}{\Delta t}
$$

A equação diferencial (5.2) e (5.1), discretizada no tempo por um esquema semiimplícito fica

$$
\begin{gathered}
\mathbf{M} \rho\left(\frac{\mathbf{u}^{n+1}-\mathbf{u}_{*}^{n}}{\Delta t}\right)-\frac{1}{R e} \mathbf{K u}^{n+1}-\mathbf{G}^{n+1}-\frac{1}{F r^{2}} \mathbf{M}_{\rho} \mathbf{g}=0 \\
\mathbf{D} \mathbf{u}^{n+1}=0
\end{gathered}
$$

Adiciona-se a hipótese de que o campo gravitacional não influencia o escoamento, podendo desta forma ser desprezado.

Utilizando um método baseado em decomposição LU para desacoplar as componentes de velocidade e pressão, podemos representar (5.4) 5.5) por um sistema da seguinte forma

$$
\left[\begin{array}{cc}
\mathbf{B} & \mathbf{0} \\
\mathbf{D} & \Delta t \mathbf{D M}_{\rho}^{-1} \mathbf{G}
\end{array}\right]\left[\begin{array}{cc}
\mathbf{I} & -\Delta t \mathbf{M}_{\rho}^{-1} \mathbf{G} \\
\mathbf{0} & \mathbf{I}
\end{array}\right]\left[\begin{array}{c}
\mathbf{u}^{n+1} \\
\mathbf{p}^{n+1}
\end{array}\right]=\left[\begin{array}{c}
\mathbf{r}^{n} \\
\mathbf{0}
\end{array}\right]+\left[\begin{array}{l}
\mathbf{b c}_{1} \\
\mathbf{b c}_{2}
\end{array}\right]
$$

onde:

$$
\begin{gathered}
\mathbf{B}=\mathbf{M}_{\rho}-\frac{\Delta t}{R e} \mathbf{K} \\
\mathbf{r}^{n}=\mathbf{M}_{\rho} \mathbf{u}_{*}^{n}
\end{gathered}
$$

e as matrizes são definidas como:

$$
\begin{array}{r}
\mathbf{M}_{\rho}=\left[\begin{array}{ccc}
\mathbf{M}_{\rho} & \mathbf{0} & \mathbf{0} \\
\mathbf{0} & \mathbf{M}_{\rho} & \mathbf{0} \\
\mathbf{0} & \mathbf{0} & \mathbf{M}_{\rho}
\end{array}\right] \\
\mathbf{K}=\left[\begin{array}{ccc}
2 \mathbf{K}_{\mathbf{x x}}+\mathbf{K}_{\mathbf{y y}}+\mathbf{K}_{\mathbf{z z}} & \mathbf{K}_{\mathbf{x y}} & \mathbf{K}_{\mathbf{x z}} \\
\mathbf{K}_{\mathbf{y x}} & \mathbf{K}_{\mathbf{x x}}+2 \mathbf{K}_{\mathbf{y y}}+\mathbf{K}_{\mathbf{z z}} & \mathbf{K}_{\mathbf{y z}} \\
\mathbf{K}_{\mathbf{z x}} & \mathbf{K}_{\mathbf{z y}} & \mathbf{K}_{\mathbf{x x}}+\mathbf{K}_{\mathbf{y y}}+2 \mathbf{K}_{\mathbf{z z}}
\end{array}\right]
\end{array}
$$


Assim, primeiro calcula-se

$$
\mathbf{B u}=\mathbf{r}^{n}+\mathbf{b c}_{1}
$$

obtendo-se o valor de $\widetilde{\mathbf{u}}$. O próximo passo é calcular a pressão e assim encontrar os valores finais das velocidades. No entanto, com o intuito de reduzir custos computacionais como tempo de processamento e uso de memória, a técnica de aproximação hidrostática foi utilizada para atualizar os valores do gradiente de pressão.

O cálculo da pressão resultante foi calculado utilizando-se como parâmetro de entrada para o simulador $2 \mathrm{D}$ a média vertical de $\widetilde{u}$ e $\widetilde{v}$ calculado em (5.7), através da seguinte integração

$$
\begin{aligned}
& \overline{\widetilde{u}}=\frac{1}{H} \int_{-h}^{\eta} \widetilde{u}_{3 D}(x, y, z, t) d z \\
& \overline{\widetilde{v}}=\frac{1}{H} \int_{-h}^{\eta} \widetilde{v}_{3 D}(x, y, z, t) d z
\end{aligned}
$$

Seja $(x, y) \in \Omega \subset \mathbb{R}^{2}$ definido como sendo os pontos no plano horizontal $z=0$ representando a superfície de referência e seja $z \in[-h, \eta]$ o ponto na direção vertical onde $h(x, y)$ representa a profundidade e $\eta(x, y, t)$ a elevação da superfície, ambas medidas a partir da superfície de referência como pode ser visto na Figura 5.2 .

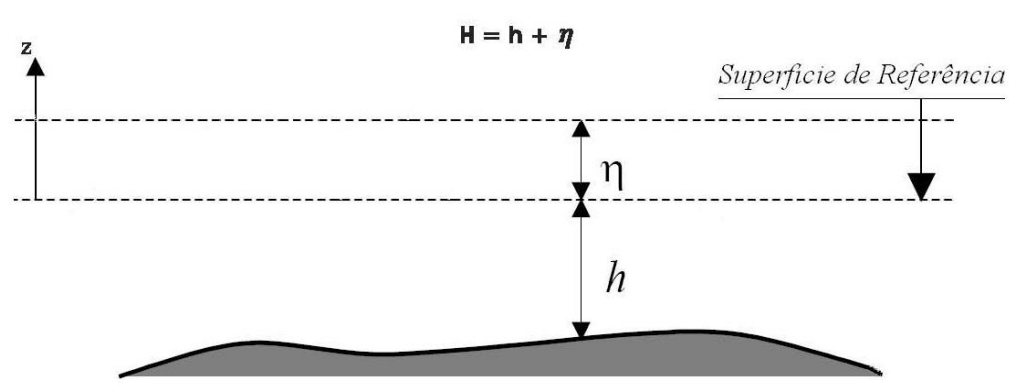

Figura 5.2: Profundidade $(h)$, elevação $(\eta)$ e superfície de referência

O domínio de interesse é a camada de largura $H=h+\eta$ confinada entre a superfície livre, descrita pela função $z=\eta(x, y, t)$ e o fundo, dado pela função $z=-h(x, y)$. Nas simulações utilizou-se $\eta=0$.

O resultados obtidos pelas equações (5.8) e (5.9) são passados para o simulador GesarSim 2D que retorna as correções das velocidades 3D utilizando-se o gradiente da pressão calculado. A pressão calculada em 2D é então propagada para os níveis inferiores da malha.

Assim, as velocidades finais podem ser atualizadas da seguinte forma: 
1. Primeiro calcula-se $\widetilde{\mathbf{u}}_{3 D}$ de

$$
\mathbf{B} \widetilde{\mathbf{u}}_{3 D}=\mathbf{r}^{n}+\mathbf{b c}_{1}
$$

2. Calcula-se as médias $\overline{\widetilde{\mathbf{u}}}$ a partir das equações (5.8) e 5.9. Ainda nesta fase, é calculada a média das velocidades nos centróides dos elementos com base nos valores das velocidades $\overline{\widetilde{\mathbf{u}}}$.

3. Calcula-se a pressão $\mathbf{p}^{n+1}$ em $2 \mathrm{D}$ por

$$
\Delta t \mathbf{D M}_{\rho}^{-1} \mathbf{G p}_{2 D}^{n+1}=-\mathbf{D} \overline{\widetilde{\mathbf{u}}}+\mathbf{b c}_{2}
$$

4. Encontra-se a velocidade final $\mathbf{u}^{n+1}$ usando

$$
\mathbf{u}^{n+1}=\widetilde{\mathbf{u}}_{3 D}+\Delta t \mathbf{M}_{\rho}^{-1} \mathbf{G}_{2 D}^{n+1}
$$

Note que o termo $\Delta t \mathbf{M}_{\rho}^{-1} \mathbf{G}_{2 D}^{n+1}$ precisa ser propagado aos vértices nos níveis inferiores da malha, a fim de se compatibilizar a dimensão dos vetores na soma das correções das velocidades.

Para garantir maior estabilidade no método de resolução das equações, os valores das velocidades nos centróides são atualizadas considerando-se esses valores no tempo anterior a cada iteração. A atualização é feita da seguinte maneira

$$
\overline{\widetilde{\mathbf{u}}}_{c}^{n+1}=\overline{\widetilde{\mathbf{u}}}_{c}^{n}+\frac{1}{3} \sum_{v=1}^{3}\left(\overline{\widetilde{\mathbf{u}}}_{v}^{n+1}-\overline{\widetilde{\mathbf{u}}}_{v}^{n}\right)
$$

onde os índices $c$ e $v$ que aparecem na equação 5.13 correspondem as velocidades no centróide e velocidades nos vértices, respectivamente.

Depois de atualizar as velocidades finais $u^{n+1}$ e $v^{n+1}$ é necessário atualizar a velocidade $w$ pela equação da continuidade, onde

$$
\frac{\partial w}{\partial z}=-\frac{\partial u}{\partial x}-\frac{\partial v}{\partial y}
$$

Integrando a equação (5.14), do fundo até um ponto qualquer $z$ de altura tem-se

$$
\int_{-h}^{z} \frac{\partial w}{\partial z}=\int_{-h}^{z}\left(-\frac{\partial u}{\partial x}-\frac{\partial v}{\partial y}\right) d z
$$


Resolvendo a integral da equação (5.15) obtém-se

$$
w-w(-h)=-\int_{-h}^{z}\left(\frac{\partial u}{\partial x}+\frac{\partial v}{\partial y}\right) d z
$$

Como $w(-h)$ é uma condição de contorno do fundo do reservatório, tem-se que $w(-h)=$ 0 , logo a velocidade $w$ é atualizada da seguinte maneira

$$
w^{n+1}=-\int_{-h}^{z}\left(\frac{\partial u^{n+1}}{\partial x}+\frac{\partial v^{n+1}}{\partial y}\right) d z
$$

onde $z \in[-h, \eta]$.

\section{Matriz de Massa Concentrada}

A matriz de massa concentrada (lumped) é uma matriz diagonal que simplifica as operações de inversão de matrizes utilizadas no método. Assim, ela foi utilizada para aproximar a matriz de massa $\mathbf{M}_{\rho}$. A matriz de massa concentrada é definida como sendo a soma dos valores de cada linha da matriz, posicionando este resultado na posição do elemento na diagonal. A inversão desse tipo de matriz é simples e rápida, quando comparada à inversão em uma matriz regular.

\subsection{Implementação do Método Semi-Lagrangeano}

A implementação do método semi-lagrangeano para o caso tridimensional onde o domínio é formado por prismas lineares segue regras específicas para a determinação das velocidades no tempo anterior. São várias as posições onde o ponto $p^{*}$ pode se localizar, como pode ser visto na Figura 5.3, que numera as possíveis variações.

Como cada posição do ponto $p *$ implica em uma interpolação diferente, a seguir os principais casos são detalhados, baseando-se nos casos numerados indicados na Figura 5.3.

1. Caso em que $p^{*}$ coincidir com algum vértice da malha. Neste caso, basta atribuir o mesmo valor das propriedades deste vértice às propriedades resultantes.

2. Caso em que $p^{*}$ localiza-se no interior da base ou do topo de um prisma. Neste caso deve-se fazer uma interpolação a partir do conhecimento de $\mathbf{u}$ nos vértices do triângulo. Assim, a função de interpolação escolhida para u é dada por

$$
\mathbf{u}=\lambda_{1} \mathbf{u}_{\mathbf{1}}+\lambda_{2} \mathbf{u}_{\mathbf{2}}+\lambda_{3} \mathbf{u}_{\mathbf{3}}
$$




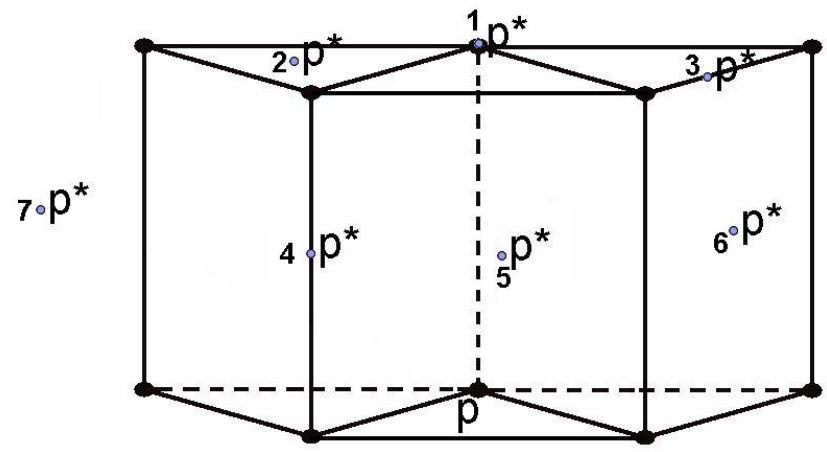

Figura 5.3: Possíveis casos de localização para $p^{*}$ no método semi-lagrangeano.

Com os valores de $\mathbf{u}_{\mathbf{1}}, \mathbf{u}_{\mathbf{2}}, \mathbf{u}_{\mathbf{3}}$ e os valores das coordenadas $(x, y)$ nos pontos 1, 2, 3 e a posição $\left(x^{*}, y^{*}\right)$, é possível encontrar os valores das constantes $\lambda_{1}, \lambda_{2}$, e $\lambda_{3}$ da seguinte maneira

$$
\begin{gathered}
1=\lambda_{1}+\lambda_{2}+\lambda_{3}, \\
x^{*}=\lambda_{1} x_{1}+\lambda_{2} x_{2}+\lambda_{3} x_{3} \\
y^{*}=\lambda_{1} y_{1}+\lambda_{2} y_{2}+\lambda_{3} y_{3}
\end{gathered}
$$

Essas expressões podem ser escritas como o seguinte sistema linear

$$
\left(\begin{array}{ccc}
1 & 1 & 1 \\
x_{1} & x_{2} & x_{3} \\
y_{1} & y_{2} & y_{3}
\end{array}\right)\left(\begin{array}{c}
\lambda_{1} \\
\lambda_{2} \\
\lambda_{3}
\end{array}\right)=\left(\begin{array}{c}
1 \\
x^{*} \\
y^{*}
\end{array}\right)
$$

cuja solução resulta nos valores para as constantes $\lambda_{1}, \lambda_{2}$, e $\lambda_{3}$.

3. Caso em que $p^{*}$ localiza-se em alguma aresta da base ou do topo de algum prisma. Neste caso faz-se uma interpolação bi-linear dos vértices na extremidade da aresta, pois agora temos duas variantes: $x$ e $y$, como segue

$$
\begin{gathered}
\alpha_{0}=\sqrt{\frac{\left(x_{v 1}-x_{v 0}\right)^{2}+\left(y_{v 1}-y_{v 0}\right)^{2}}{\left(x_{v 1}-x_{v 0}\right)^{2}+\left(y_{v 1}-y_{v 0}\right)^{2}}} \\
\alpha_{1}=1-\alpha_{0} \\
p^{*}=\alpha_{0} V_{0}+\alpha_{1} V_{1}
\end{gathered}
$$

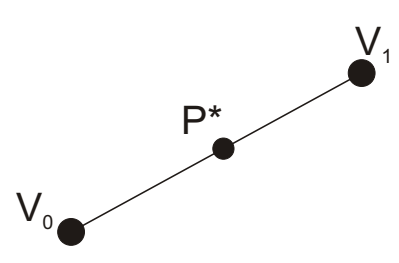


onde $x_{v i}$ corresponde ao valor da coordenada $x$ do vértice $i$ e $y_{v i}$ corresponde ao valor da coordenada $y$ do vértice $i$.

4. O caso em que $p^{*}$ localiza-se em alguma aresta vertical do prisma. Neste caso faz-se uma interpolação linear dos vértices na extremidade da aresta, da seguinte maneira:

$$
\begin{aligned}
\alpha_{0} & =\frac{z_{v_{1}}-z_{p^{*}}}{z_{v_{1}}-z_{v_{0}}} \\
\alpha_{1} & =1-\alpha_{0} \\
p^{*} & =\alpha_{0} V_{0}+\alpha_{1} V_{1}
\end{aligned}
$$

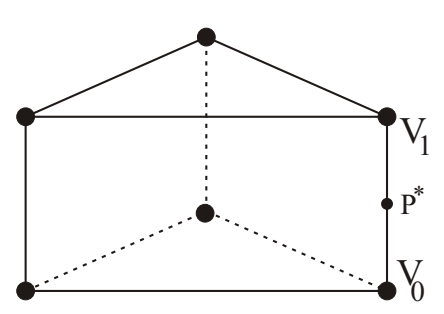

Esquema de interpolação para aresta vertical.

5. Caso em que $p^{*}$ localiza-se no interior de algum prisma. Após recuperados os seis vértices correspondentes, a seguinte técnica foi realizada para encontrar os parâmetros necessários para a interpolação.

Sabendo-se que qualquer ponto em um espaço tridimensional pode ser escrito em termos de quaisquer outros três vértices (que podem formar a origem do espaço cartesiano), pode-se realizar uma projeção do prisma em um triângulo para determinar os parâmetros para estes três vértices e em seguida considerar a componente vertical z para determinar todos os seis parâmetros necessários. Formalmente, considere $\left(x_{1}, y_{1}\right),\left(x_{2}, y_{2}\right),\left(x_{3}, y_{3}\right)$ as componentes $x, y$ dos três vértices da base ou topo do prisma, e $\left(x_{p}, y_{p}\right)$ as componentes $x, y$ de $p^{*}$. Considere também que os níveis são definidos por $\operatorname{deep}[i]$ e $\operatorname{deep}[i+1]$, onde $\operatorname{deep}[i]>\operatorname{deep}[i+1]$. A projeção é feita da seguinte maneira. Primeiro calcula-se os coeficientes da interpolação considerando-se a projeção de $p^{*}$ no triângulo da base ou topo:

$$
A=\left(\begin{array}{ccc}
1 & 1 & 1 \\
x_{1} & x_{2} & x_{3} \\
y_{1} & y_{2} & y_{3}
\end{array}\right), b=\left(\begin{array}{c}
1 \\
x_{p}^{*} \\
y_{p}^{*}
\end{array}\right)
$$

Em seguida,

$$
\left(A * A^{T}\right) L=b
$$




$$
\lambda=A^{T} * L
$$

Desta forma, calcula-se os coeficientes $\lambda$ referentes aos vértices do triângulo da projeção. Os coeficientes $\lambda$ do triângulo da base são os mesmos do triângulo do topo do prisma. Assim, é necessário agora considerar a coordenada $z$ para a interpolação vertical, e assim temos os coeficientes finais:

$$
\begin{gathered}
\alpha[i+1]=\frac{\operatorname{deep}[i]-z_{p}^{*}}{\operatorname{deep}[i]-\operatorname{deep}[i+1]} \\
\alpha[i]=1-\alpha[i+1]
\end{gathered}
$$

onde $i$ é o $i d$ dos vértices. Portanto,

$$
\lambda=[\alpha[i] * \lambda ; \alpha[i+1] * \lambda]
$$

resulta nos seis coeficientes necessários.

6. Caso em que $p^{*}$ localiza-se no interior de uma face lateral de um prisma. A solução segue a mesma idéia para o caso do ponto no interior do prisma. Primeiro projeta-se a face em uma reta, encontrando-se dois parâmetros por uma combinação linear, e em seguida considera-se a coordenada z para gerar os 4 parâmetros necessários.

7. Caso em que $p^{*}$ localiza-se fora da malha. Neste caso, deve-se considerar duas possibilidades. Uma delas é o ponto $p^{*}$ localizar-se acima do topo ou abaixo da base do domínio, e outra é a localização entre o topo e a base do domínio. Na primeira possibilidade, é necessário realizar uma intersecção entre a reta $\left\{p, p^{*}\right\}$ e o plano da base (ou do topo caso $p *$ estiver acima dele) e, verificando se o ponto da intersecção pertence à malha.

Desta forma, é possível saber em que ponto a equação da reta interceptou o plano. A partir daí, existem alguns casos a considerar. Se a intersecção reta-plano não gerou um ponto pertencente à malha, temos o caso em que $p^{*}$ interceptou uma face lateral de um prisma. Neste caso, primeiro encontra-se a face correspondente, e realiza-se uma nova intersecção de reta e plano da face para saber onde é o ponto de intersecção. A partir daí, se o ponto de interseccção encontra-se no interior de uma face, faz-se uma combinação linear dos quatros vértices pertencentes a esta face (note que este procedimento também vale para a segunda possibilidade supracitada). A 
estratégia aqui segue o mesmo procedimento feito no interior do prisma, só que agora para 4 vértices apenas. Primeiro projeta-se a face em uma reta, encontrando-se dois parâmetros por uma combinação linear, e em seguida considera-se a coordenada $z$ para gerar os 4 parâmetros necessários. Mas se o ponto de interseccção encontrase em um vértice ou aresta, deve-se tomar as medidas necessárias que repetem os procedimentos demonstrados nos itens acima.

\subsection{Considerações Finais}

Este capítulo apresentou as estratégias utilizadas no Simulador GesarSim 3D e alguns detalhes de implementação.

Para o cálculo da pressão utilizou-se uma aproximação hidrostática. Esta estratégia se mostrou vantajosa em termos de custos computacionais, pois a pressão é calculada na tampa e propagada para os níveis inferiores, assim como o termo que corrige as atualizações das velocidades.

Foram apresentados alguns detalhes do método semi-lagrangeano, o qual possui implementação particular ao domínio de representação, que é responsável pela aproximação dos termos convectivos das equações. A montagem das matrizes também foi discutida, assim como a utilização da aproximação lumping para a aproximar a matriz de massa, o que resultou em um procedimento mais atrativo em nível de custos computacionais. 


\section{Capítulo \\ 6 \\ Validação e Resultados Numéricos}

Neste capítulo são apresentados os resultados numéricos obtidos a partir do simulador GesarSim 3D, que é a implementação em linguagem C++, desenvolvida neste trabalho para o caso de escoamento de fluidos em domínios tridimensionais. Primeiramente, é feita uma validação do modelo mostrando a convergência do método desenvolvido e em seguida são apresentados alguns resultados obtidos a partir da ferramenta desenvolvida.

\subsection{Introdução}

Os resultados aqui apresentados são simulações de escoamento de fluidos newtonianos incompressíveis para problemas tridimensionais na presença de contornos rígidos. A validação consiste em verificar um perfil parabólico que se desenvolve durante a simulação. O perfil deve ser comparado com uma solução analítica conhecida. Outra forma de validação consiste em verificar a conservação de massa do sistema, verificando assim se o modelo produz equações conservativas.

São apresentados alguns casos relacionados a problemas tridimensionais. O primeiro deles é o caso de escoamento em um canal retangular aberto. Em seguida foi simulado o caso de escoamento em um degrau e por fim o caso de escoamento com inclusão de escadas no domínio.

Os experimentos foram executados em um computador com a seguinte configuração: 
- Processador Intel Pentium 4 HT 2.8GHz 1Mb L2, 1 Gb RAM e S.O. Linux Slackware 9.0 .

\subsection{Escoamento em um Canal}

Nesta seção, são apresentados testes de simulação de escoamentos de fluido em um canal onde se verifica o comportamento das velocidades do fluido durante um enchimento gradativo do canal. Considera-se que o reservatório já está cheio no início da simulação com uma velocidade prescrita na entrada do canal. Primeiramente, é feita uma validação numérica do método desenvolvido com condição de escoamento totalmente desenvolvido imposta na entrada de fluido no canal. Em seguida são apresentados resultados numéricos obtidos em um canal retangular com um perfil de velocidade uniforme na entrada do canal.

\subsubsection{Validação Numérica - Perfil de Velocidade Parabólico na Entrada do Canal}

Uma possível validação para o modelo tridimensional é avaliar a solução resultante do Simulador GesarSim 3D com uma solução analítica. Uma solução que pode ser comparada com a solução resultante da simulação é a solução analítica da equação de Poisson

$$
-\nabla^{2} u=f
$$

A solução coincide com a correspondente da componente de velocidade na direção $x$ de um escoamento desenvolvido e estacionário em um canal aberto quadrado com altura $H=\frac{L}{2}$, ou seja, a metade do lado $a=L$, considerando que a superfície é uma linha de simetria.

A solução analítica é dada por

$$
\begin{gathered}
u(y, z)=\frac{\left(1-y^{2}\right)}{2}-\frac{16}{\pi^{3}} \sum_{k=1, k \text { impar }}^{\infty}\left\{\frac{\sin (k \pi(1+y) / 2)}{k^{3} \sinh (k \pi)}\right. \\
\times(\sinh (k \pi(1+z) / 2)+\sinh (k \pi(1-z) / 2))\}
\end{gathered}
$$

Um domínio quadrado de lado $L=2 m$ e altura $H=1 m$ foi definido para esta simulação. Neste caso, condições de não deslizamento foram impostas na parede do domínio 
para as componentes de velocidade $u, v$ e $w$. A pressão tem valor nulo na saída do canal. A componente de velocidade na direção $z$ tem valor nulo nos níveis superior e inferior do domínio. A condição para escoamento totalmente desenvolvido definida na entrada de fluido é dada pela equação (6.2). A geometria do domínio é apresentada na Figura 6.1, que mostra as dimensões dos lados do canal e a entrada de fluido no mesmo. A malha utilizada nesta simulação pode ser observada na Figura 6.2, localizada no plano $x y$ da Figura 6.1.

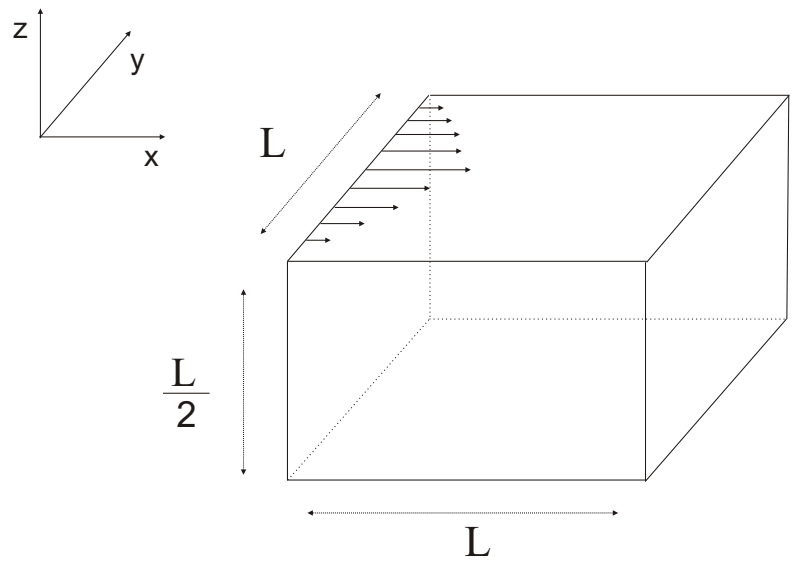

Figura 6.1: Geometria do domínio, o escoamento é da esquerda para a direita.

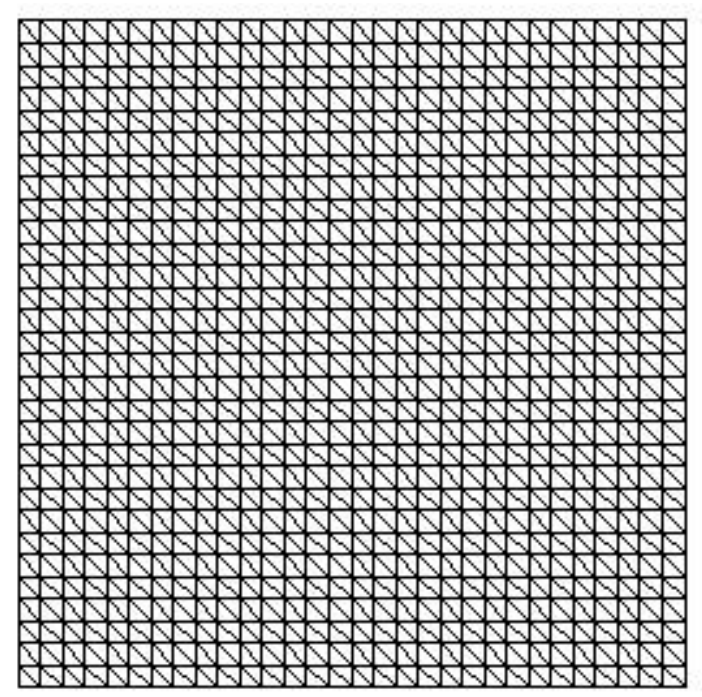

Figura 6.2: Malha utilizada na simulação vista pelo eixo z.

Para este caso utilizou-se seis pontos na direção $z$, ou seja, cinco camadas de prismas, sendo que a malha possui um total de 4000 elementos prismáticos, e consequentemente 800 
triângulos formados na malha superficial, mostrada na Figura 6.2.

Quando se atinge o estado estacionário, os valores das velocidades ao longo do canal são os mesmos que os definidos na entrada de fluido, e desta forma, pode-se comparar os resultados numéricos com a solução analítica dada pela expressão 6.2). Após o desenvolvimento do fluido dentro do canal, as comparações podem ser realizadas a partir de um modelo para simulação.

O modelo para esse escoamento é:

- Dimensão do domínio: $2.0 \mathrm{~m} \times 2.0 \mathrm{~m}$ x $1.0 \mathrm{~m}$;

- Largura da região de entrada de fluido: $2.0 \mathrm{~m}$;

- Viscosidade : $1.00 \mathrm{Ns} / \mathrm{m}^{2}$. Densidade $1.0 \mathrm{~kg} / \mathrm{m}^{3}$;

- Malha superficial: 21 x 21 pontos, total de elementos : 4000;

- Parâmetros de escala : $L=2.0 \mathrm{~m}$ e $H=1.0 \mathrm{~m}$;

- $\quad$ Número de Reynolds = 10;

A Figura 6.3 mostra o resultado numérico obtido pela simulação. A região considerada para a comparação do resultado numérico com a solução analítica neste caso, é a saída de fluido do canal. Como se pode observar, o resultado numérico obtido é bastante semelhante à solução analítica, onde o erro máximo para esta simulação foi da ordem de $10^{-3}$.

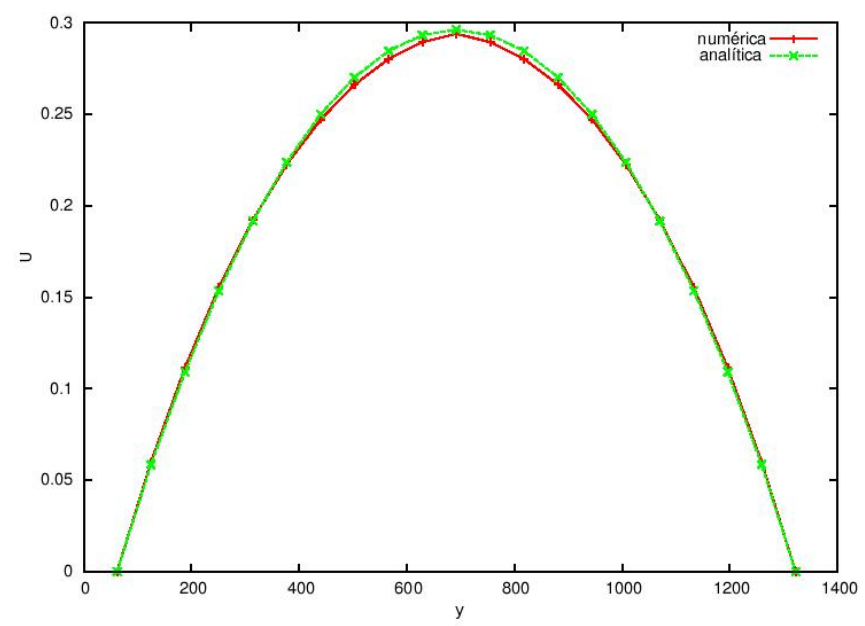

Figura 6.3: Comparação do resultado obtido pelo simulador GesarSim 3D com a solução analítica. 


\subsubsection{Conservação de Massa do Sistema}

É muito importante que o modelo desenvolvido produza equações conservativas, pois toda massa que entra no sistema deve sair do sistema, ou seja, a vazão de entrada deve ser a mesma vazão de saída. Desta forma, para este caso em particular, foram realizados alguns testes variando-se o número de elementos na malha, a fim de verificar a consistência do método desenvolvido. Considera-se uma velocidade prescrita e com perfil do tipo parabólico na entrada do canal. As condições iniciais e de contorno são as mesmas impostas para a validação numérica do método.

O teste de conservação de massa resume-se em analisar a vazão de entrada do sistema frente à vazão de saída do fluido, após o escoamento atingir um estado estacionário. A Tabela 6.1 mostra os resultados obtidos, onde variou-se o número de camadas de prismas $N C P$ (refinando assim a malha lógica tridimensional considerando-se a componente $z$ ) além da dimensão da malha no plano $x y$, utilizando $R e=10$. O número de elementos formados no domínio são 8000, 12000, 27000, 20000 e 54000 elementos, respectivamente às malhas com 11,16, 26 e 31 níveis, descritas na Tabela 6.1. Observe que o número de níveis $N$ do domínio é dado pela fórmula $N=N C P+1$ e o número de elementos $N e$ no domínio é dado pela fórmula $N e=N C P * N C e l l s$, onde $N C P$ é o número de camadas de prismas e $N C e l l s$ é o número de células (triângulos) formadas na malha superficial, número este fornecido pela estrutura de dados utilizada.

O erro absoluto é definido como sendo o módulo da diferença entre a massa na entrada e a massa na saída. Ainda na tabela, nota-se que os valores obtidos se aproximam, e o erro diminui conforme aumenta-se o número de pontos no domínio, seja pelo número de pontos por camada tanto pelo aumento no número de camadas, o que torna o sistema mais consistente. Portanto pode-se considerar que o sistema conserva massa em um nível aceitável.

\begin{tabular}{|ccccc|}
\hline NCP & Dimensão da Malha & Massa na Entrada & Massa na Saída & Erro Absoluto \\
\hline 10 & $21 \times 21$ & 0.380285 & 0.401344 & 0.021059 \\
15 & $21 \times 21$ & 0.392294 & 0.401434 & 0.009140 \\
15 & $31 \times 31$ & 0.392903 & 0.401612 & 0.008709 \\
25 & $21 \times 21$ & 0.396297 & 0.401466 & 0.005169 \\
30 & $31 \times 31$ & 0.392903 & 0.393608 & 0.000705 \\
\hline
\end{tabular}

Tabela 6.1: Teste da conservação de massa para o escoamento em um canal. 


\subsubsection{Perfil de Velocidade Uniforme na Entrada do Canal - Ge- ometria e Condições de Contorno}

Neste caso, utilizou-se um perfil de velocidade uniforme na entrada do canal e para a realização das simulações foi utilizado um domínio que representa um canal tridimensional. A geometria pode ser observada na Figura 6.5. Primeiramente, são analisados os resultados obtidos em um corte horizontal feito na metade da altura do canal e na saída de fluido do canal. Posteriormente, são analisados os resultados obtidos na superfície do domínio, especificamente na saída de fluido do canal. A malha superficial utilizada nas simulações pode ser vista na Figura 6.4.

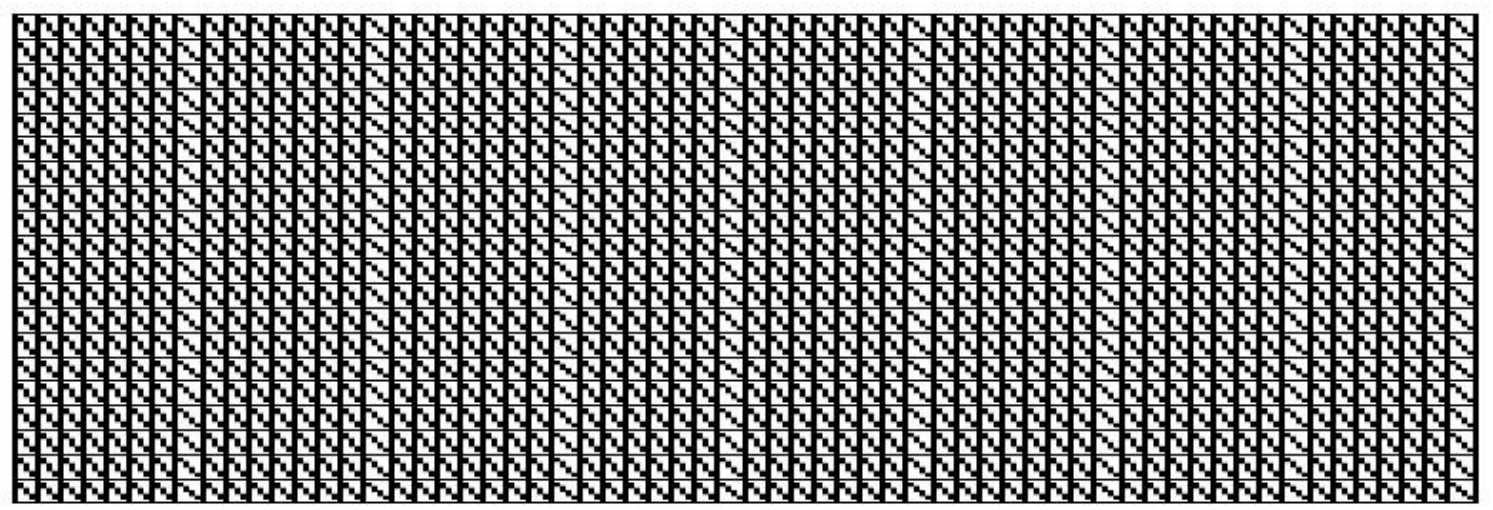

Figura 6.4: Malha utilizada nas simulações do caso de escoamento em um canal com vista pelo eixo $z$.

A especificação do modelo para esse escoamento é definido como:

- Dimensão do domínio: $3.0 \mathrm{~m} \times 1.0 \mathrm{~m} \times 1.0 \mathrm{~m}$;

- Largura da região de entrada de fluido: $1.0 \mathrm{~m}$;

- Viscosidade : $1.00 \mathrm{Ns} / \mathrm{m}^{2}$;

- Densidade : $1.0 \mathrm{~kg} / \mathrm{m}^{3}$;

- Parâmetros de escala: $L=1.0 \mathrm{~m}$ e $H=1.0 \mathrm{~m}$;

- Número de Reynolds : 1.0, 10, 100, 1000 e 10000. 


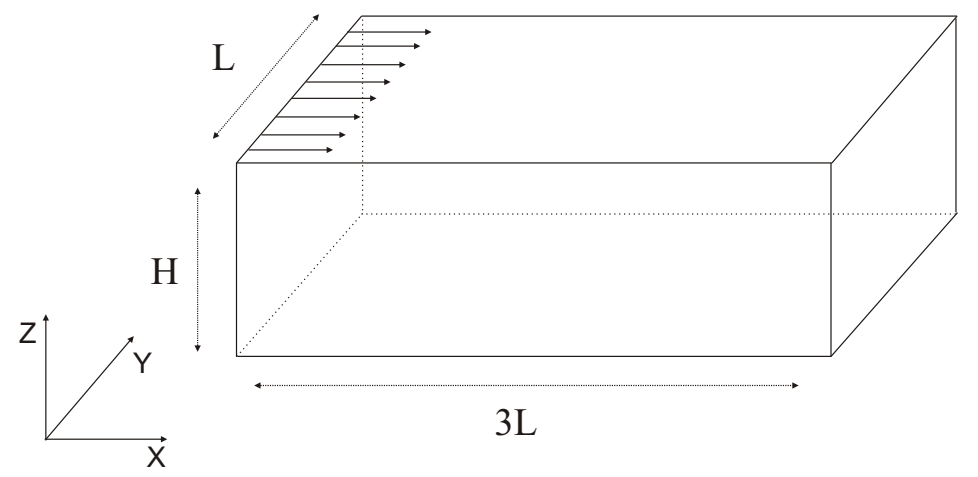

Figura 6.5: Domínio para um escoamento em um canal tridimensional. O escoamento é da esquerda para a direita.

Os resultados aqui mostrados são obtidos na saída de fluido do canal, quando os valores das velocidades atingem seu estado estacionário. As condições de contorno podem ser vistas na Figura 6.6, um exemplo em duas camadas de prismas. No início da simulação o canal está completamente cheio com uma velocidade constante para a componente na direção $x$ igual a $u=1.0 \mathrm{~m} / \mathrm{s}$ aplicada na entrada do canal, as componentes de velocidades $v$ e $w$ valem zero na entrada do canal. Na saída de fluido do canal a pressão vale zero. A componente de velocidade na direção $z$ vale zero nos níveis referentes à superfície e ao fundo do domínio. As figuras apresentadas em todas as simulações correspondem aos campos de velocidades e pressão. Foram realizadas simulações utilizando-se cinco camadas de prismas com 12400 elementos prismáticos lineares e vinte camadas de prismas com 496000 elementos.

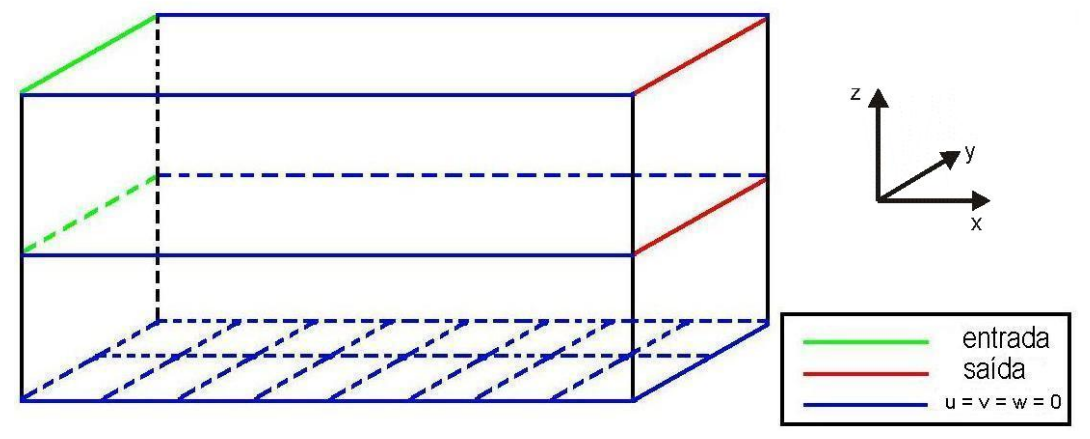

Figura 6.6: Condições de contornos utilizadas nas simulações.

O primeiro caso analisado apresenta uma simulação com seis pontos na direção z (5 
camadas de prismas) e $R e=10$. Neste caso foi utilizado um número de Reynolds relativamente baixo, por este fato as forças viscosas exercem maior influência no escoamento tornando-o tipicamente viscoso. O comportamento resultante pode ser observado na Figura 6.7. obtido no corte horizontal na metade do domínio. Em seguida, a Figura 6.8 mostra resultados da mesma simulação, utilizando-se $R e=100$. Nesta última simulação pode-se observar um escoamento diferenciado em relação ao anterior, onde há uma diminuição da espessura da camada limite observado na componente de velocidade na direção $x$, devido à diminuição da influência dos termos viscosos, relacionada ao número de Reynolds mais elevado.

Um segundo caso analisado é mostrado na Figura 6.9, onde são mostrados os resultados da simulação de escoamento com vinte camadas de prismas (vinte e um pontos na direção $z)$ e $R e=10$. Neste caso, pode-se observar que a componente de velocidade na direção $x$ possue valores inferiores em relação a simulação da Figura 6.7. Este fato ocorre pelo aumento no número de pontos na direção $z$, onde há uma maior influência desta componente sendo possível também verificar uma mudança no comportamento da componente de velocidade nesta direção. A Figura 6.10 apresenta o comportamento da mesma simulação, mas agora com $R e=1000$. Com o número de Reynolds alto o escoamento torna-se predominantemente convectivo e a espessura da camada limite da componente de velocidade na direção $x$ se torna mais fina.

O terceiro caso analisado é observar o comportamento da velocidade na superfície do domínio, conforme o número de Reynolds é variado. As Figuras 6.11, 6.12 e 6.13 apresentam o comportamento da componente de velocidade na direção $x$, utilizando-se um domínio com vinte camadas de prismas e $R e=1,100$ e 1000, respectivamente. Como o resultado da simulação é obtido na saída do canal e na superfície do domínio, pode-se observar que conforme aumenta-se o número de Reynolds, aumenta-se o tempo para o escoamento tornar-se totalmente desenvolvido e por isso a figura apresenta perfil de velocidade atenuado.

A Figura 6.14 mostra os perfis da componente de velocidade na direção $x$ para $R e=$ 10000. Para este resultado observa-se grandes oscilações nos valores de velocidade da componente na direção $x$ tanto no meio do canal como na saída de fluido do canal, neste caso não houve convergência do método.

Uma maneira de medir a convergência do método é medir o resíduo do método do gradiente conjugado nas iterações. A Figura 6.15 mostra este efeito para o caso da simulação com cinco camadas de prismas. 


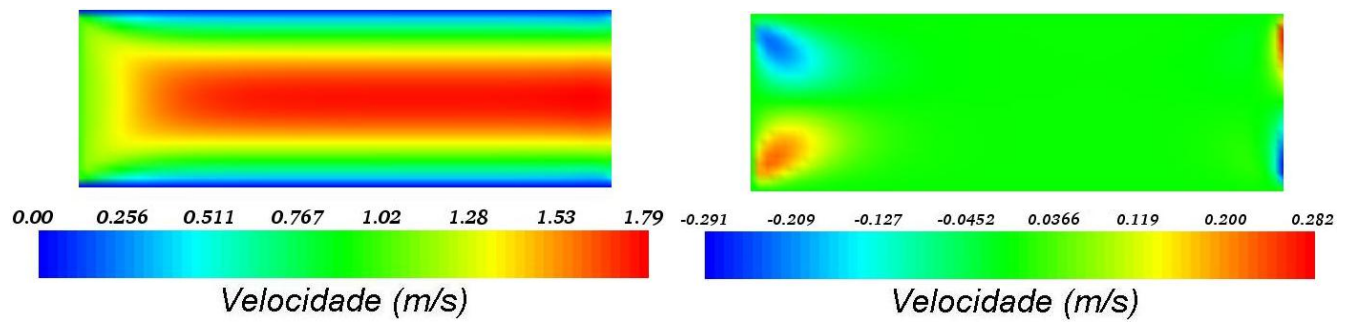

(a)

(b)

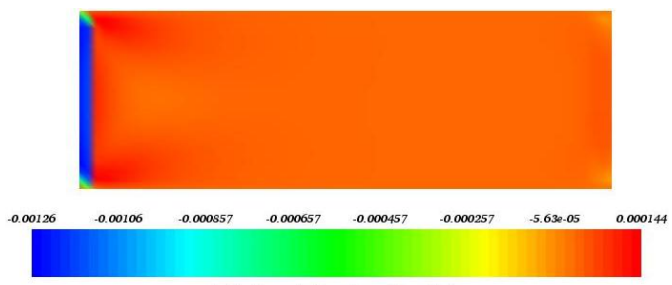

Velocidade $(\mathrm{m} / \mathrm{s})$

(c)

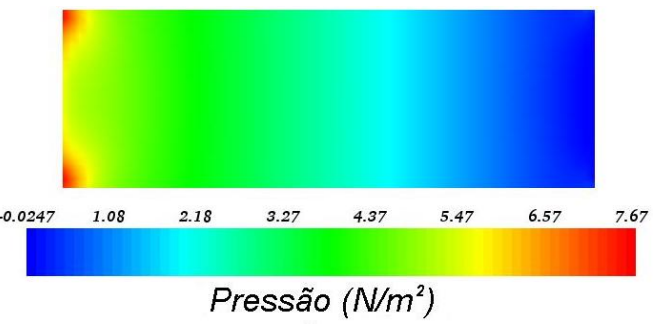

(d)

Figura 6.7: Simulação de escoamento em um canal com $R e=10$ e cinco camadas de prismas, a) componente de velocidade na direção $x, \mathrm{~b}$ ) componente de velocidade na direção $y$, c) componente de velocidade na direção $z$ e d) pressão.

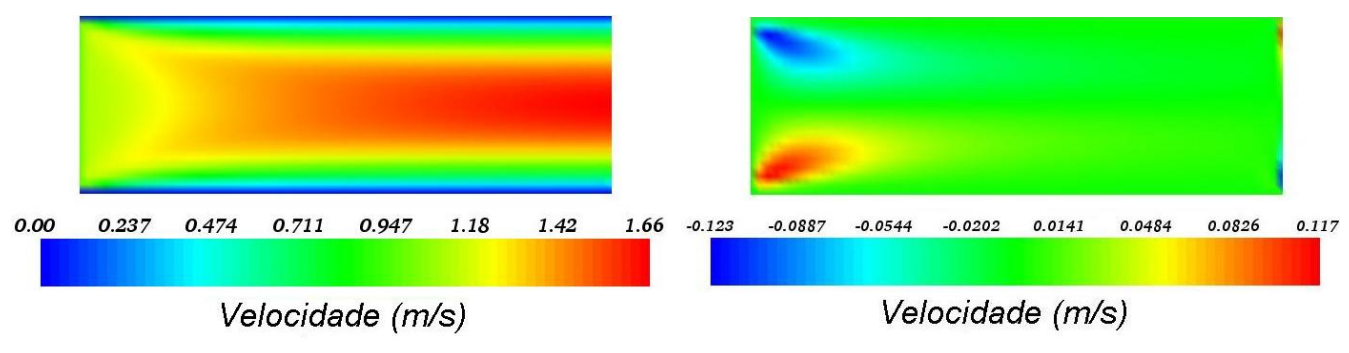

(a)

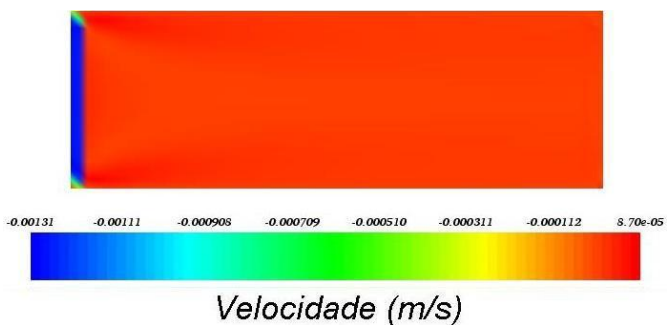

(c) (b)

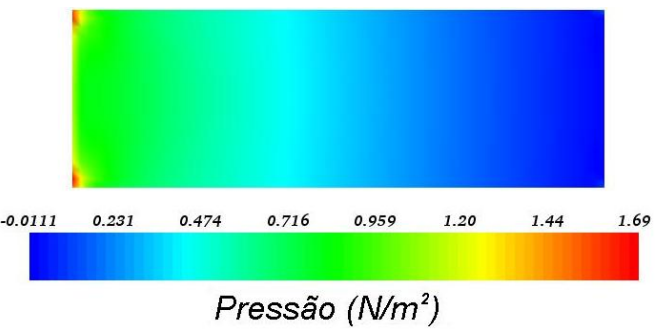

(d)

Figura 6.8: Simulação de escoamento em um canal com $R e=100$ e cinco camadas de prismas, a) componente de velocidade na direção $x, \mathrm{~b}$ ) componente de velocidade na direção $y$, c) componente de velocidade na direção $z$ e d) pressão. 


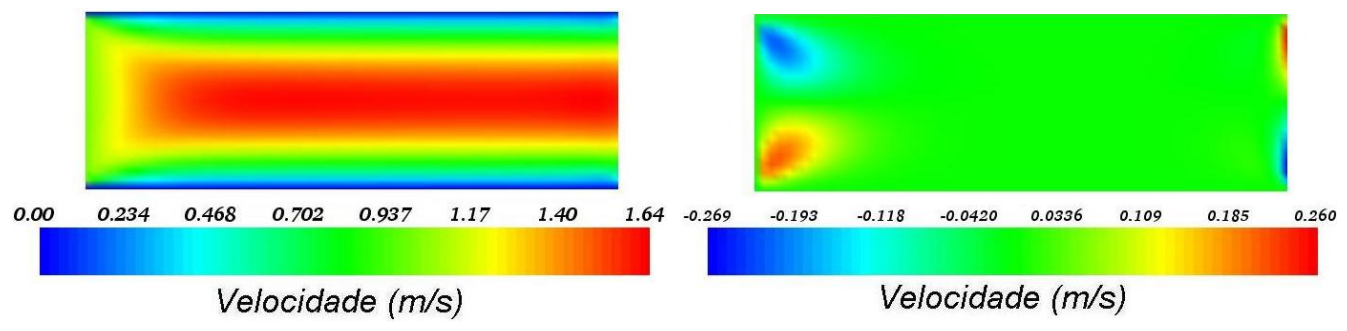

(a)

(b)

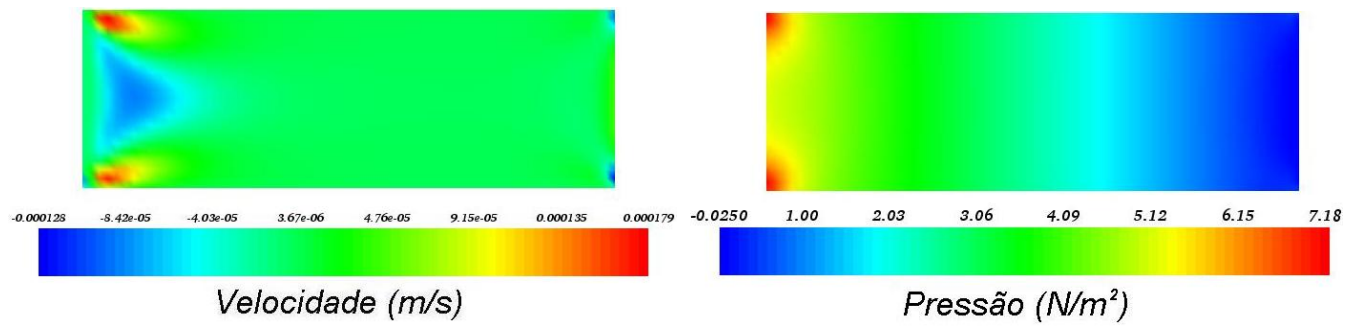

(c)

(d)

Figura 6.9: Simulação de escoamento em um canal com $R e=10$ e vinte camadas de prismas, a) componente de velocidade na direção $x, \mathrm{~b}$ ) componente de velocidade na direção $y$, c) componente de velocidade na direção $z$ e d) pressão.

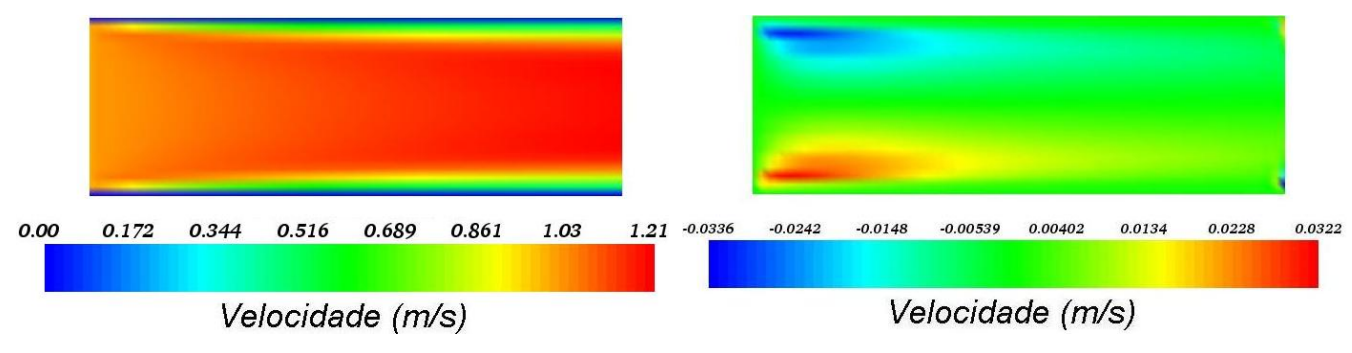

(a)

(b)

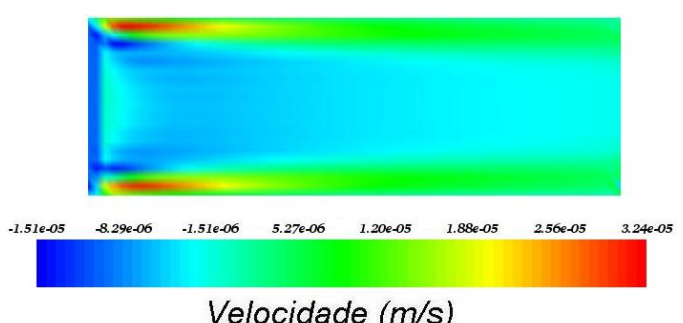

Velocidade $(\mathrm{m} / \mathrm{s})$

(c)

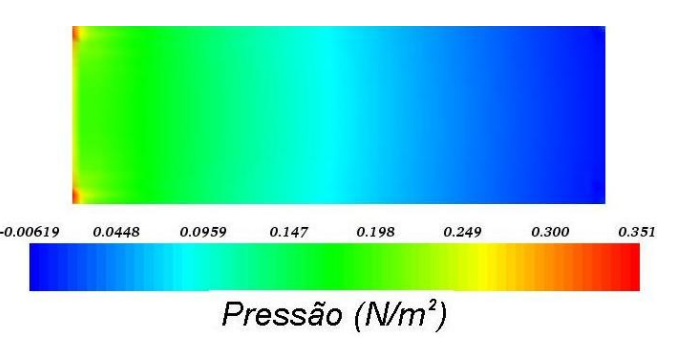

(d)

Figura 6.10: Simulação de escoamento em um canal com $R e=1000$ e vinte camadas de prismas, a) componente de velocidade na direção $x$, b) componente de velocidade na direção $y$, c) componente de velocidade na direção $z$ e d) pressão. 


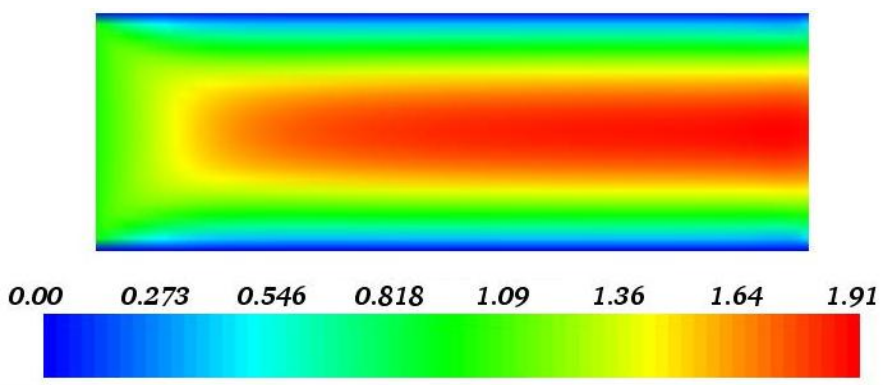

Velocidade $(\mathrm{m} / \mathrm{s})$

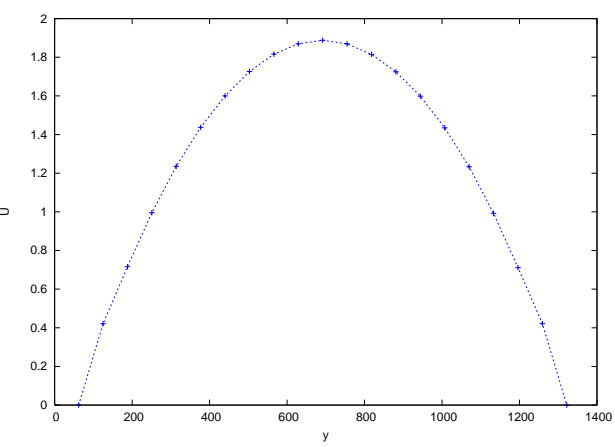

Figura 6.11: Simulação numérica do escoamento em um canal com perfil reto: campo da componente de velocidade na direção $x \operatorname{com} R e=1$ e vinte camadas de prismas.
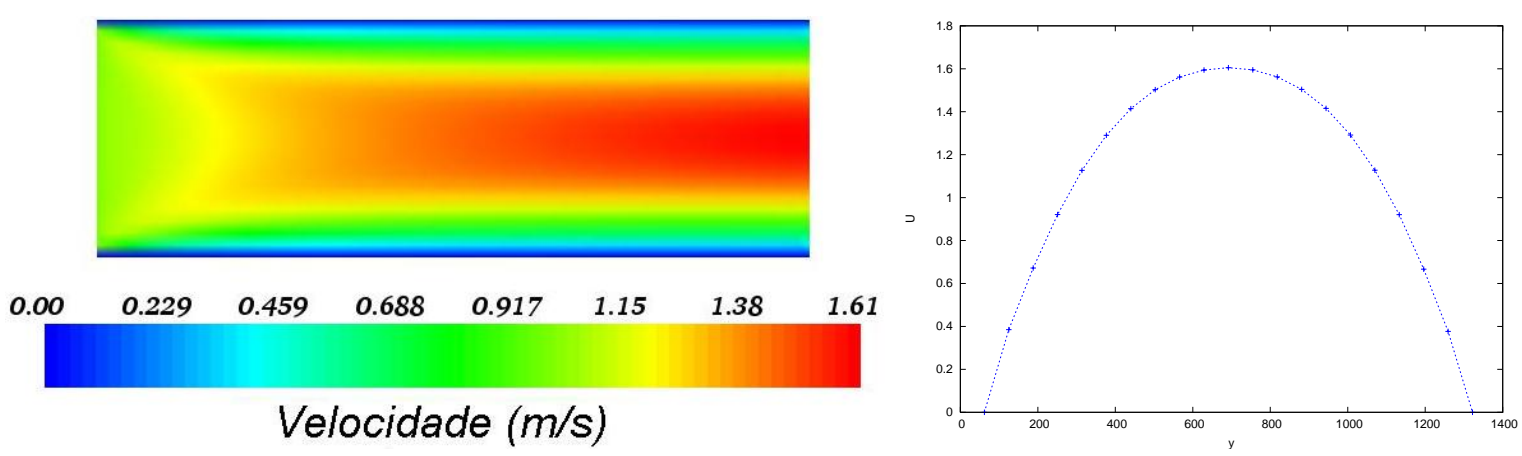

Figura 6.12: Simulação numérica do escoamento em um canal com perfil reto: campo da componente de velocidade na direção $x \operatorname{com} R e=100$ e vinte camadas de prismas.
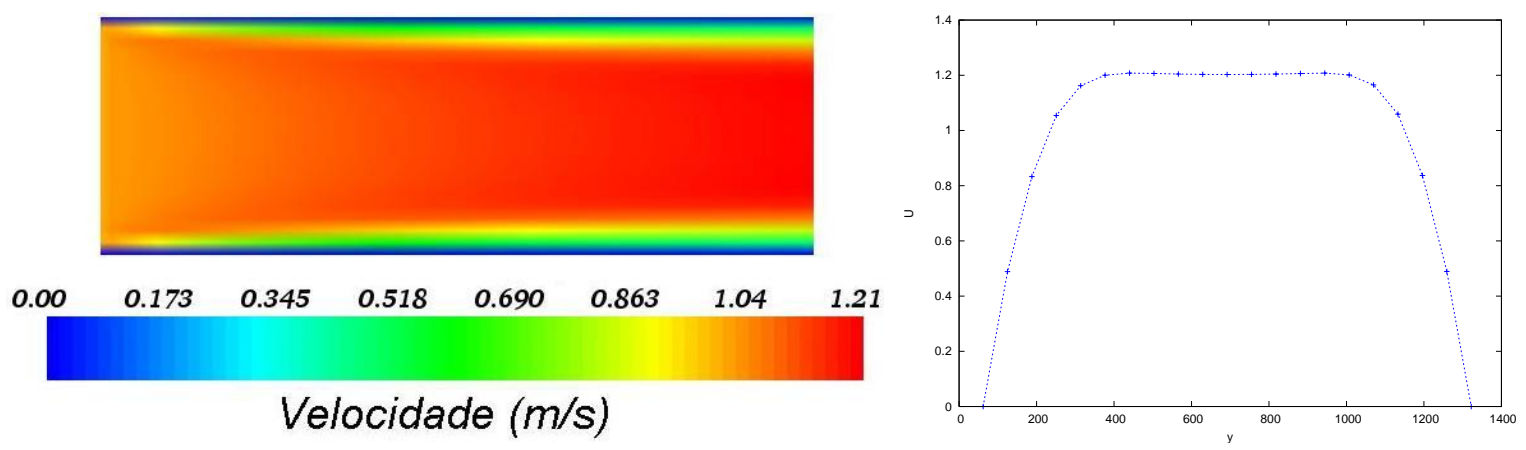

Figura 6.13: Simulação numérica do escoamento em um canal com perfil reto: campo da componente de velocidade na direção $x$ com $R e=1000$ e vinte camadas de prismas. 


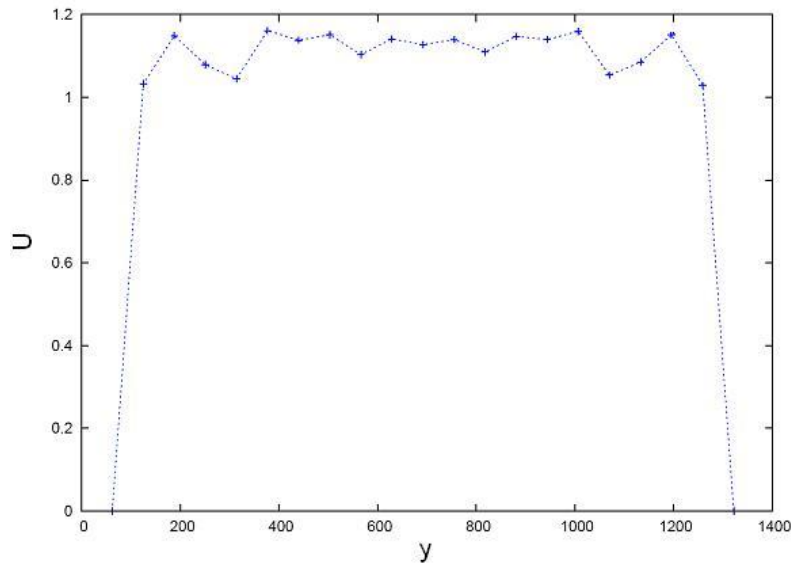

(a)

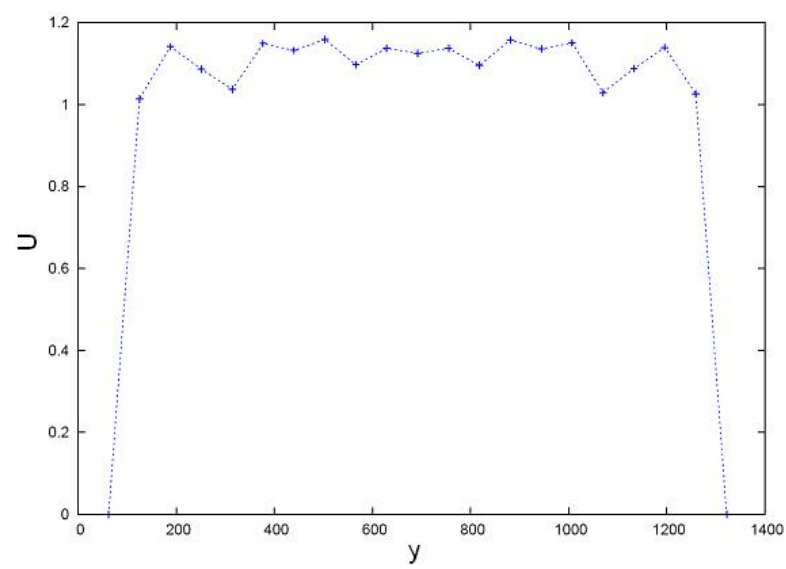

(b)

Figura 6.14: Resultado obtido na superfície do domínio: campo da componente de velocidade na direção $x$ com $R e=10000$ e cinco camadas de prismas, a) resultado obtido no meio do canal, b) resultado obtido na saída de fluido do canal

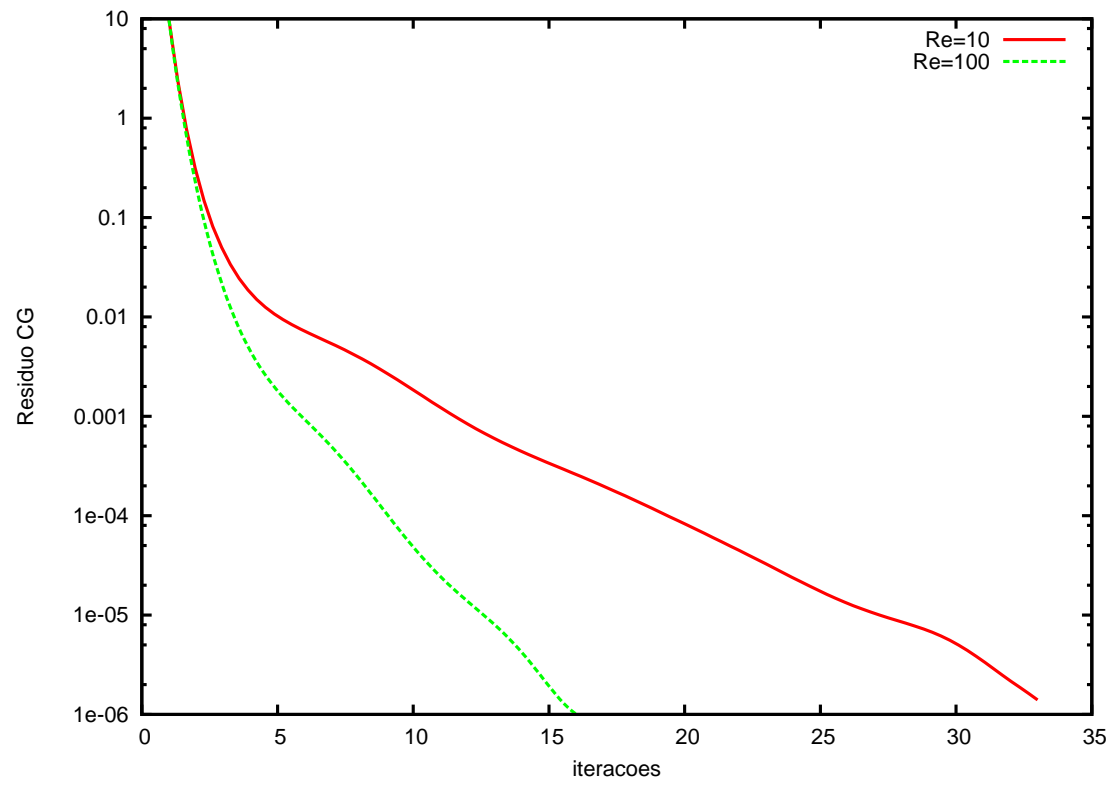

Figura 6.15: Resíduo do método de gradiente conjugado nas iterações. 


\subsection{Escoamento em um Degrau}

A simulação de escoamento incompressível em um degrau é investigado nesta seção. Este caso, apresenta em seu escoamento alto grau de complexidade, pois há presença de camada limite em desenvolvimento, zona de recirculação, descolamento e recolamento do escoamento após o degrau. Primeiramente são definidas a geometria e condições de contorno e em seguida são apresentados os resultados de simulação.

\subsubsection{Geometria e Condições de Contorno}

A geometria do problema é mostrada na Figura 6.17. A simulação foi feita utilizando como condições de entrada no canal $u=1.0 \mathrm{~m} / \mathrm{s}, v$ e $w$ com valor zero. Foi utilizado um total de 12400 elementos prismáticos nas simulações. Os resultados foram obtidos na superfície do domínio e na saída de fluido do canal. A malha superficial utilizada para esta simulação pode ser vista na Figura 6.16.

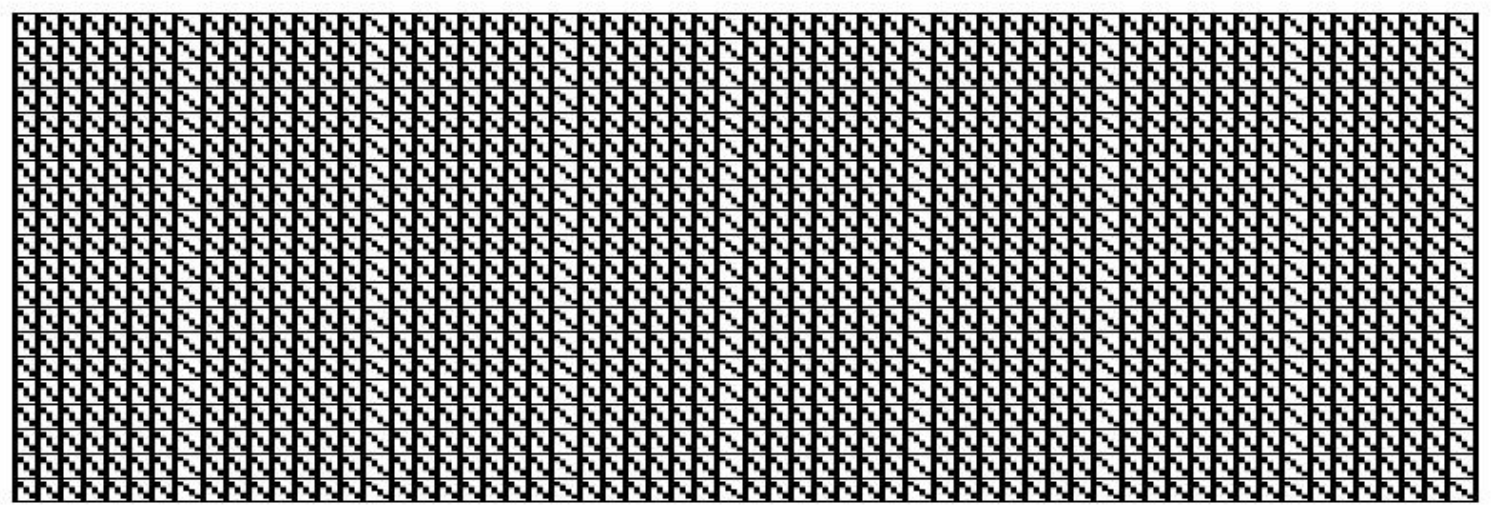

Figura 6.16: Malha superficial utilizada nas simulações do caso de escoamento em um degrau com vista pelo eixo $z$.

O modelo para esse escoamento é

- Dimensão do domínio: $3.0 m \times 1.0 m$ x $1.0 \mathrm{~m}$;

- Largura da região de entrada de fluido: $0.5 \mathrm{~m}$;

- Viscosidade : $1.00 \mathrm{Ns} / \mathrm{m}^{2}$;

- Densidade : $1.0 \mathrm{~kg} / \mathrm{m}^{3}$; 
- Parâmetros de escala: $L=1.0 \mathrm{~m}$ e $H=1.0 \mathrm{~m}$;

- Número de Reynolds : 1.0,10, 100 e 1000.

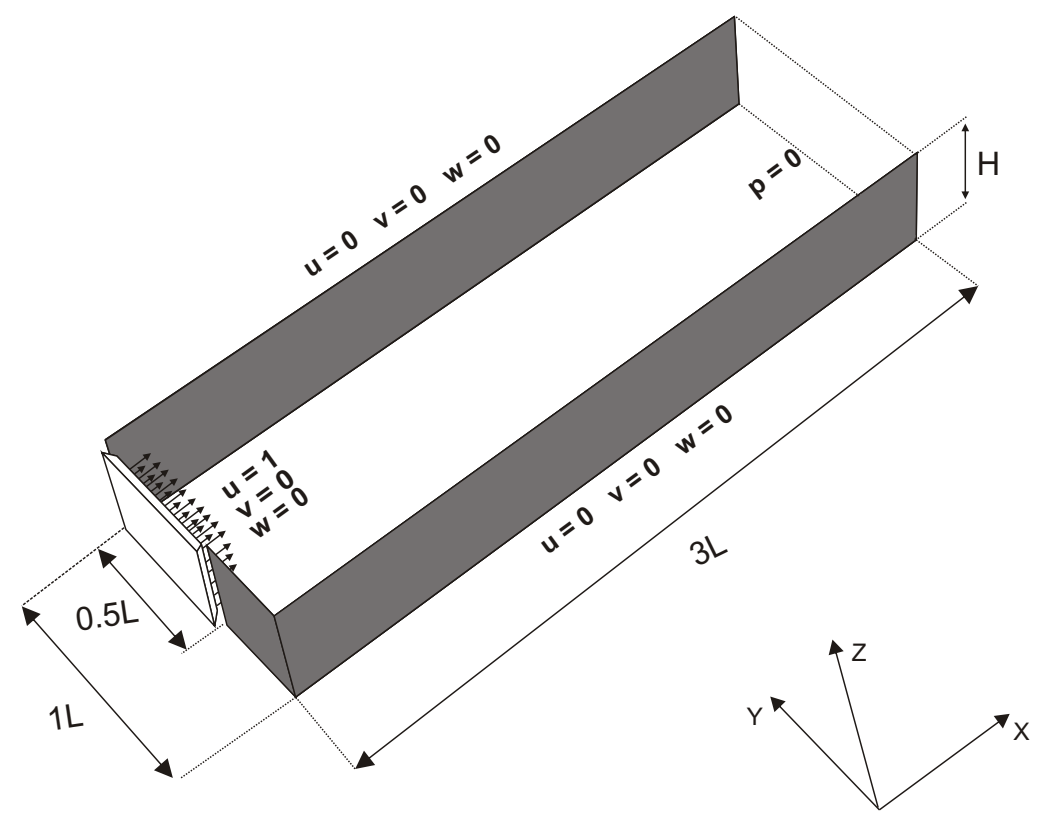

Figura 6.17: Geometria e condições de contorno para escoamento em um degrau. A região de entrada de fluido é dada por $0.5 \mathrm{~L}$

Neste caso foram impostas condições de não deslizamento nas paredes do domínio para as componentes de velocidade $u, v$ e $w$. A pressão vale zero na saída do canal. Os dois primeiros casos foram considerados números de Reynolds relativamente baixos, $R e=1 \mathrm{e}$ $R e=10$. Com o número de Reynolds baixo, tem-se que os termos viscosos das equações de Navier-Stokes exercem maior influência sobre os termos convectivos no escoamento. Neste caso o escoamento expande-se imediatamente após o obstáculo. A camada limite da componente de velocidade na direção $x$ possui baixo gradiente e por esse fato ela se torna espessa como pode ser observado na Figura 6.19

Na simulação apresentada pela Figura 6.22 o número de Reynolds ainda é considerado baixo, portanto ainda caracteriza um escoamento tipicamente viscoso, mas a camada limite da componente de velocidade na direção $x$ não apresenta as mesmas características como no caso da Figura 6.19. É possível observar um início de recirculação abaixo do degrau.

Nas simulações apresentadas nas Figuras 6.25 e 6.28, observa-se escoamento pouco caracterizado pela viscosidade. O número de Reynolds alto diminui a influência do termo 
viscoso e podem ser observadas na componente de velocidade na direção $x$ valores negativos, onde está localizado o degrau o que implica na formação de recirculações no escoamento como era esperado. Verifica-se que dependendo do número de Reynolds, o comprimento da região de recirculação aumenta, retardando o recolamento do escoamento.

As Figuras 6.31 e 6.32 mostram o escoamento contendo todos os níveis do domínio com $R e=1$ e $R e=100$, para os campos das componentes de velocidade em todas as direções e também para pressão.

A Figura 6.33 apresenta a simulação da componente de velocidade na direção $x$, contendo todos os níveis do domínio de modo a observar a simulação na entrada do canal variando-se o número de Reynolds.

A Figura 6.18 mostra o número de iterações e o resíduo obtido pelo método de gradiente conjugado utilizado nas soluções dos sistemas lineares resultantes da discretização das equações de Navier-Stokes.

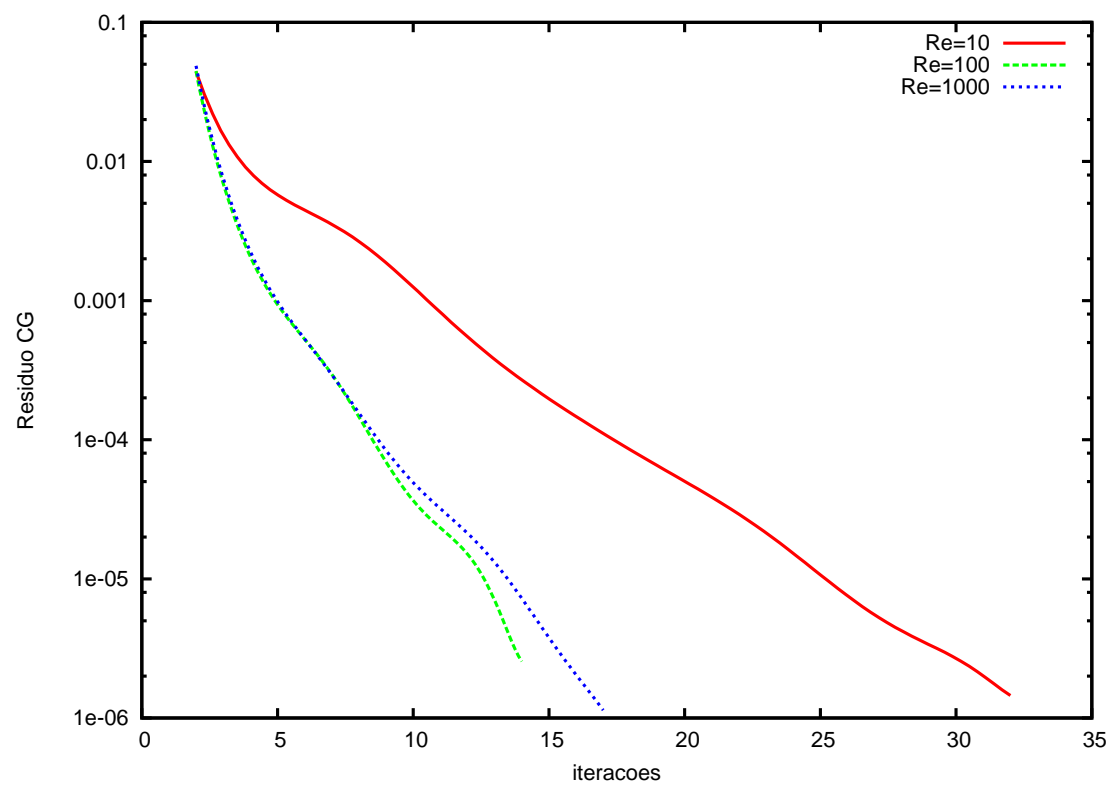

Figura 6.18: Resíduo do método de gradiente conjugado nas iterações variando o número de Reynolds. 

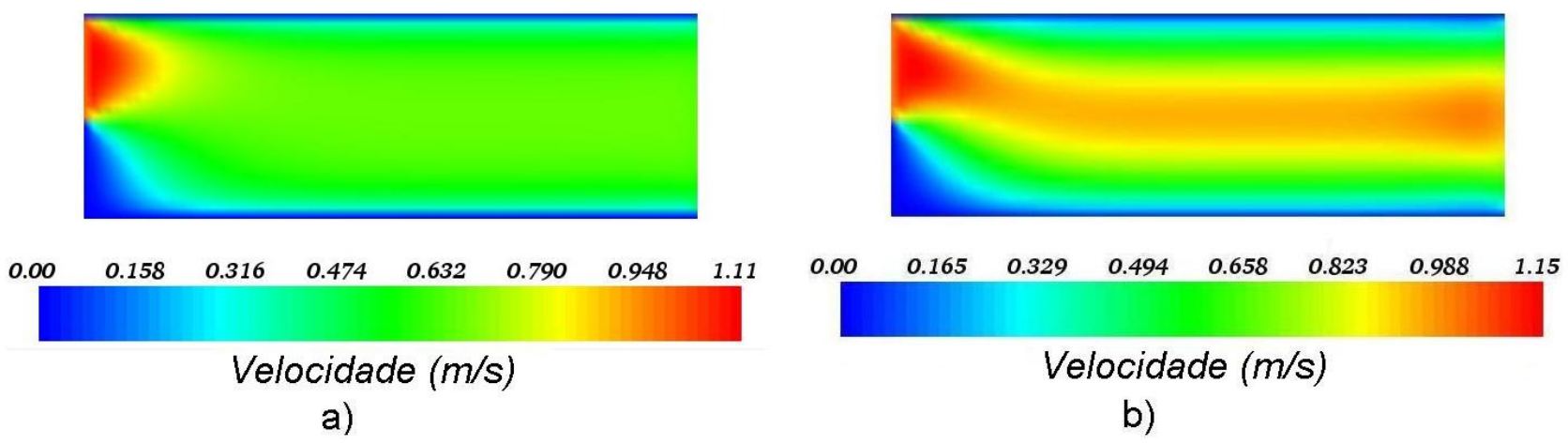

Figura 6.19: Campo da componente de velocidade na direção $x \operatorname{com} R e=1$ : a) estado inicial, b) estado avançado.
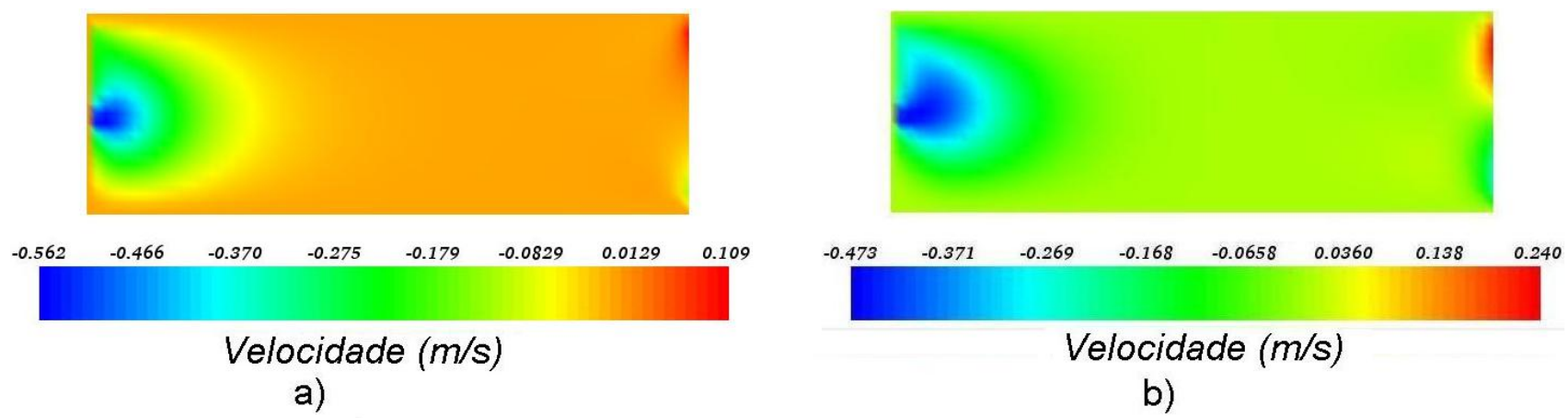

Figura 6.20: Campo da componente de velocidade na direção y com $R e=1$ : a) estado inicial, b) estado avançado.

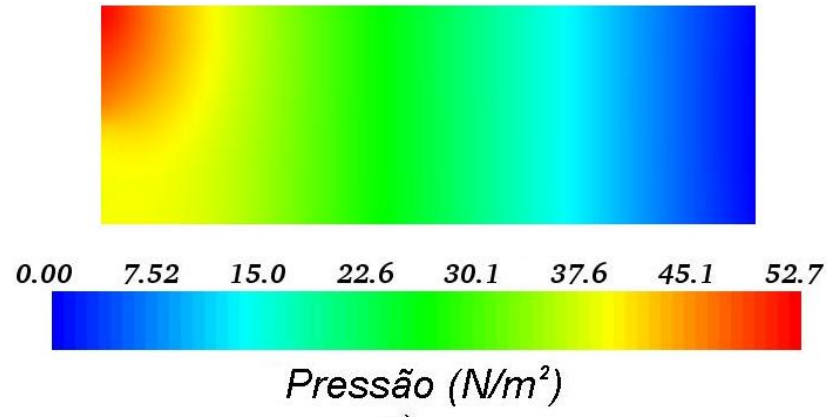

a)

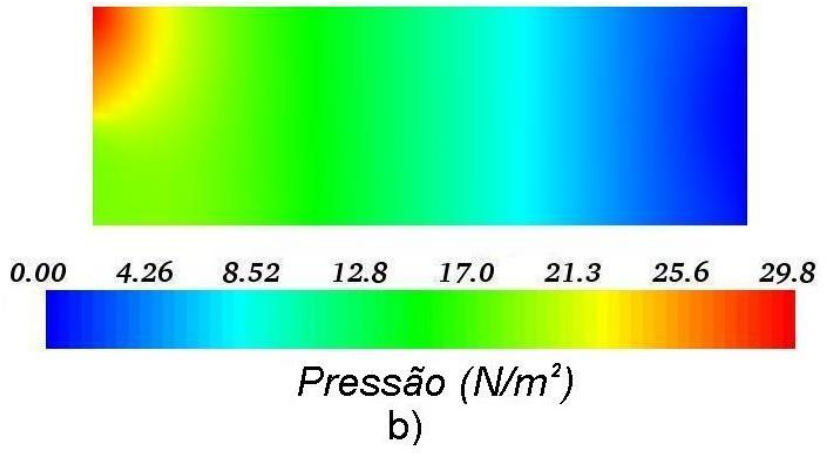

Figura 6.21: Pressão resultante da simulação com $R e=1$ : a) estado inicial, b) estado avançado. 


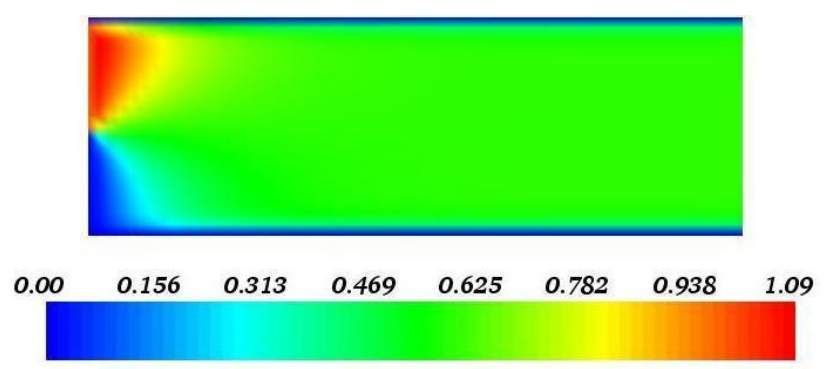

Velocidade $(\mathrm{m} / \mathrm{s})$

a)
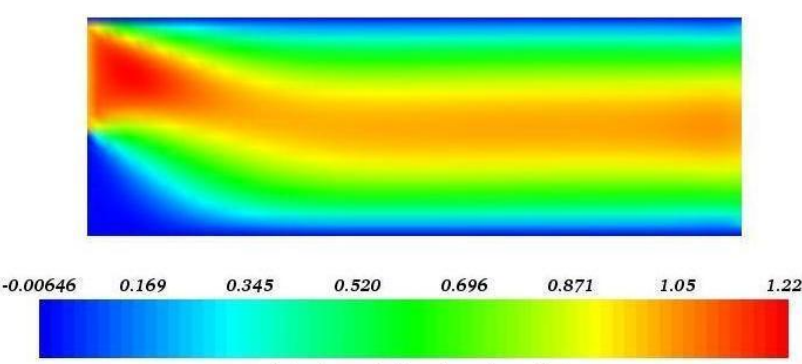

Velocidade $(\mathrm{m} / \mathrm{s})$

b)

Figura 6.22: Campo da componente de velocidade na direção $x \operatorname{com} R e=10$ : a) estado inicial, b) estado avançado.
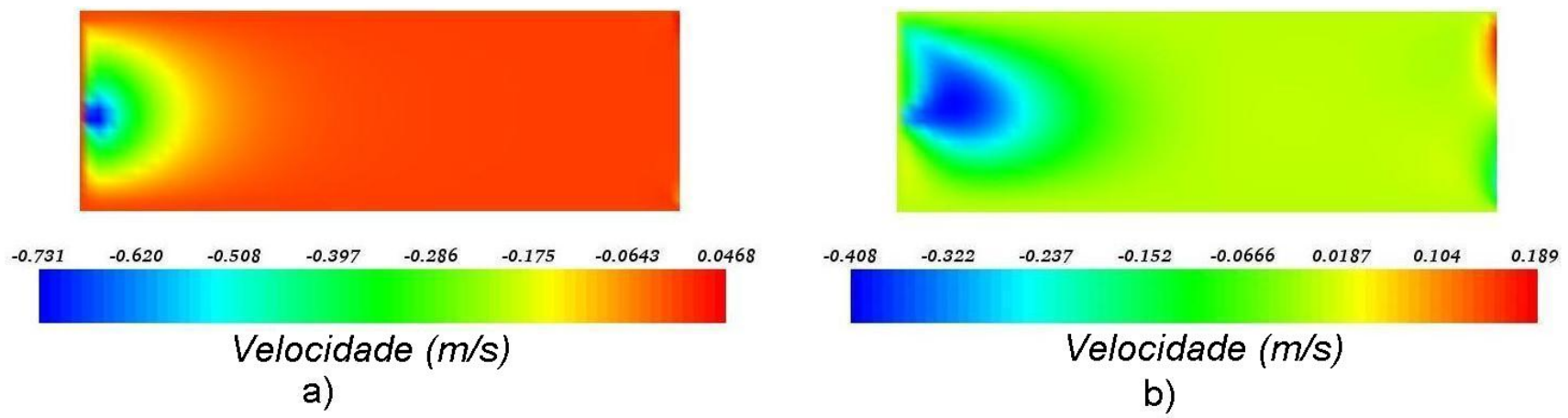

Figura 6.23: Campo da componente de velocidade na direção $y$ com $R e=10$ : a) estado inicial, b) estado avançado.
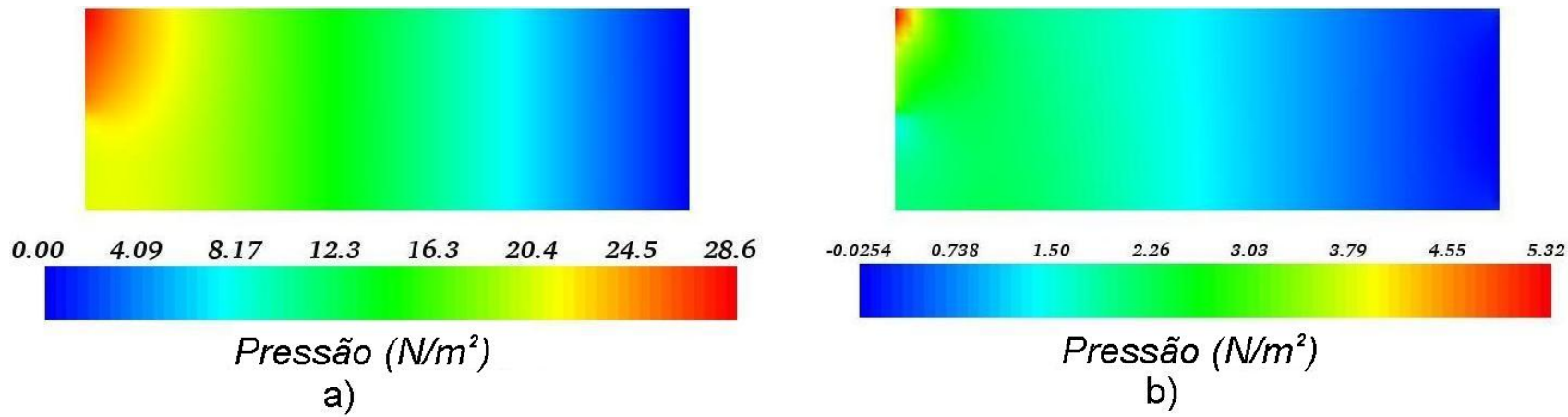

Figura 6.24: Pressão resultante da simulação com $R e=10$ : a) estado inicial, b) estado avançado. 

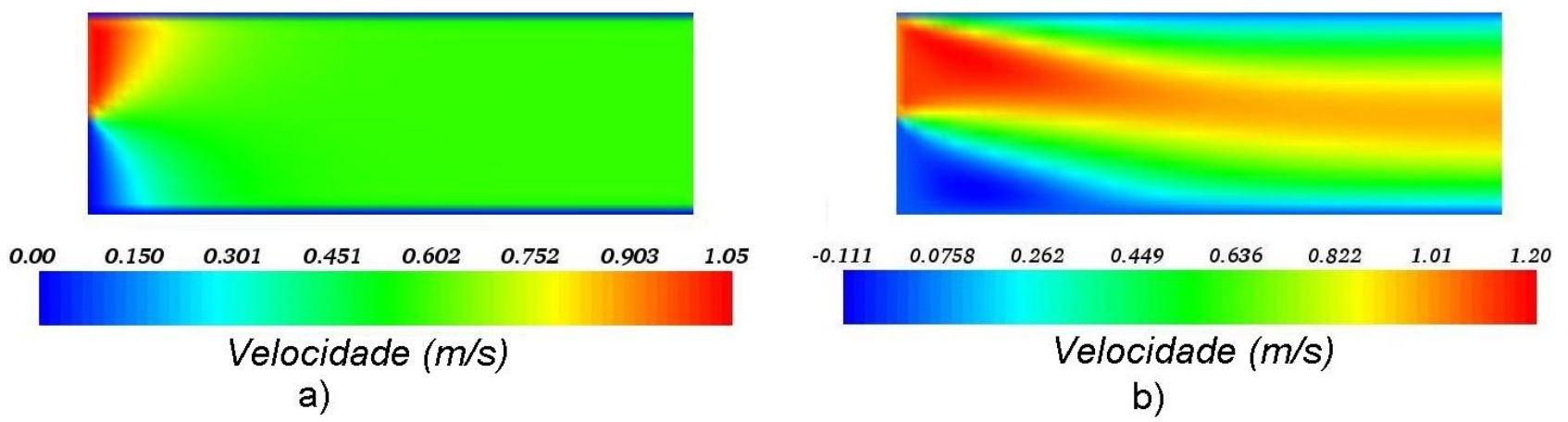

Figura 6.25: Campo da componente de velocidade na direção $x$ com $R e=100$ : a) estado inicial, b) estado avançado.
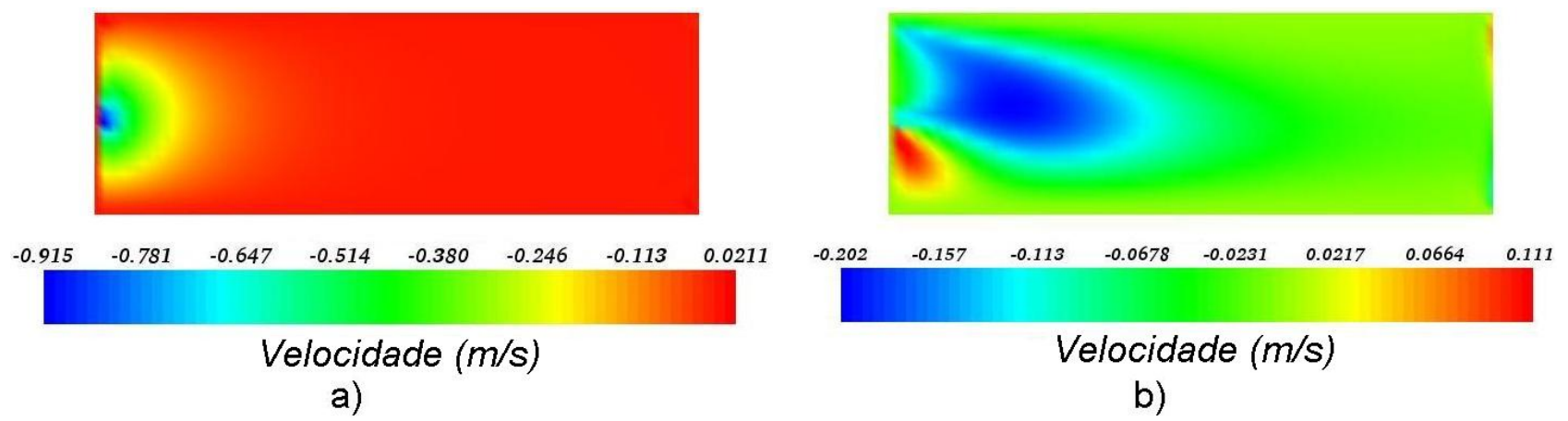

Figura 6.26: Campo da componente de velocidade na direção $y$ com $R e=100$ : a) estado inicial, b) estado avançado.

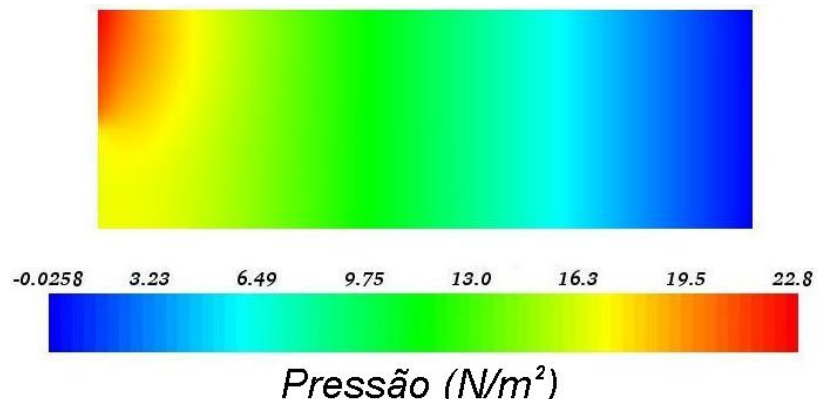

a)

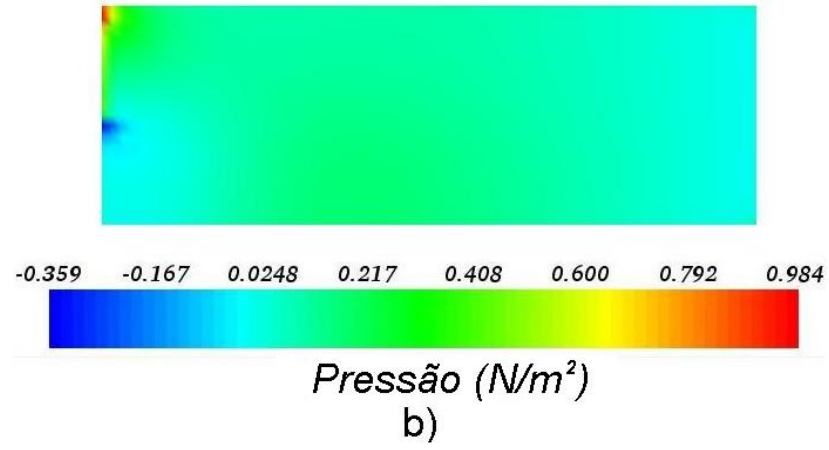

Figura 6.27: Pressão resultante da simulação com $R e=100$ : a) estado inicial, b) estado avançado. 


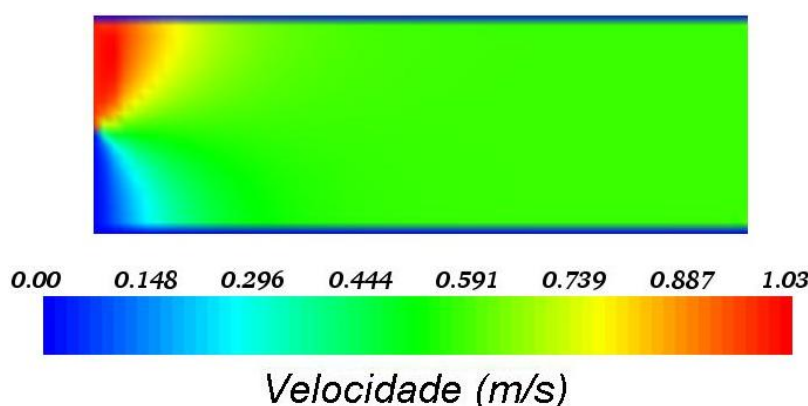

a)

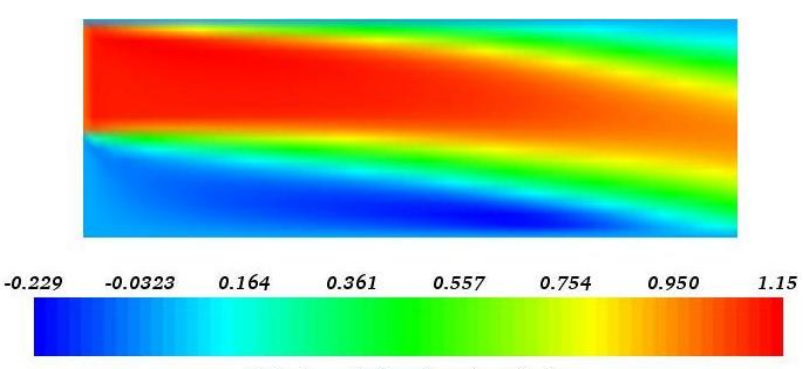

Velocidade $(\mathrm{m} / \mathrm{s})$

b)

Figura 6.28: Campo de velocidade na direção $x \operatorname{com} R e=1000$ : a) estado inicial, b) estado avançado.
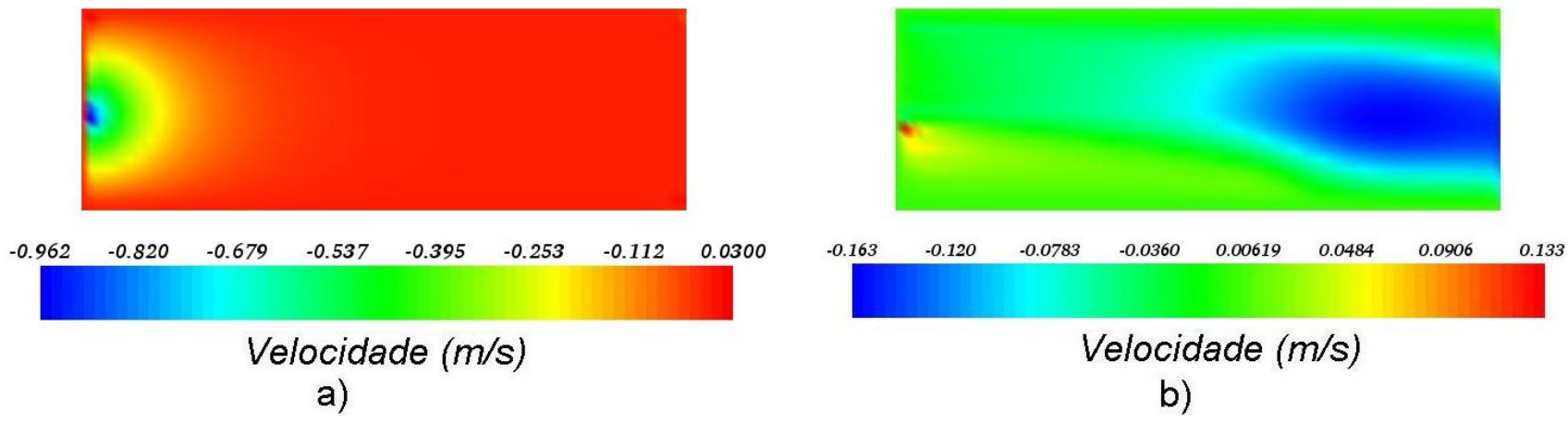

Figura 6.29: Campo da componente de velocidade na direção $y$ com $R e=1000$ : a) estado inicial, b) estado avançado.

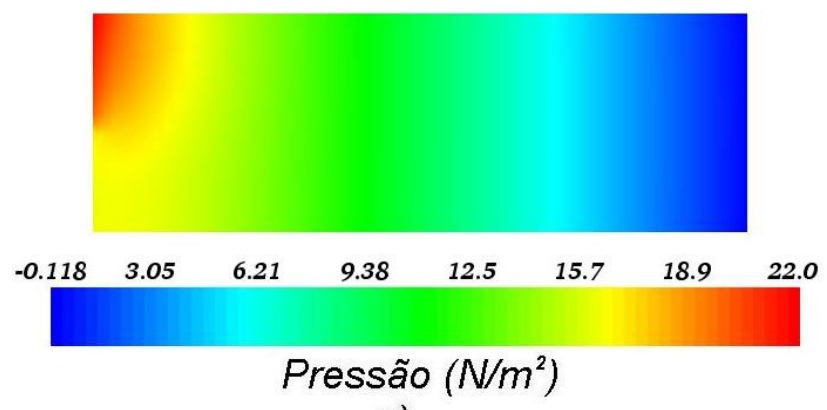

a)

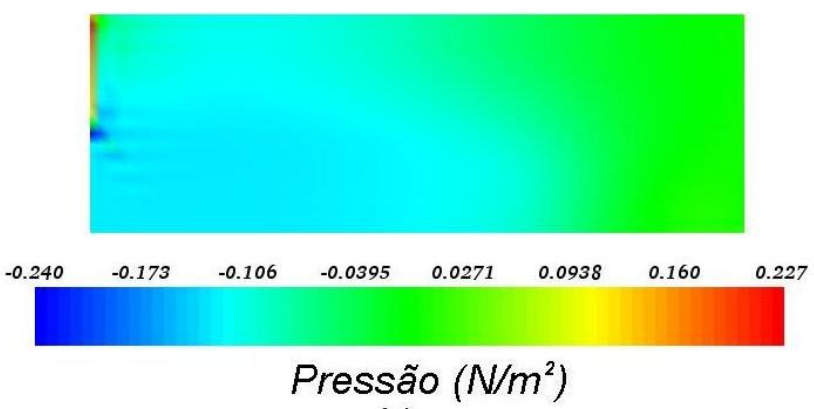

b)

Figura 6.30: Pressão resultante da simulação com $R e=1000$ : a) estado inicial, b) estado avançado. 


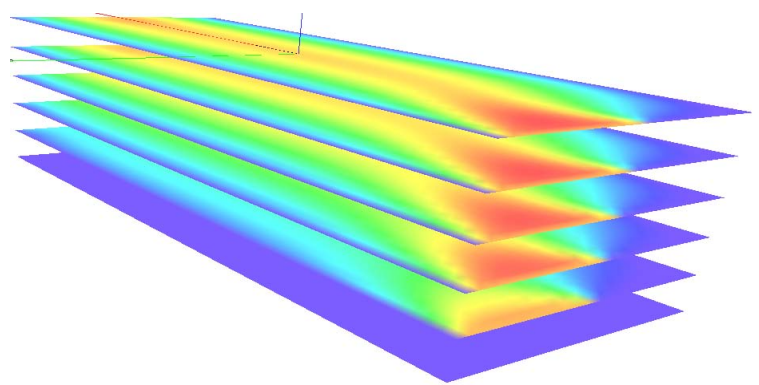

(a)

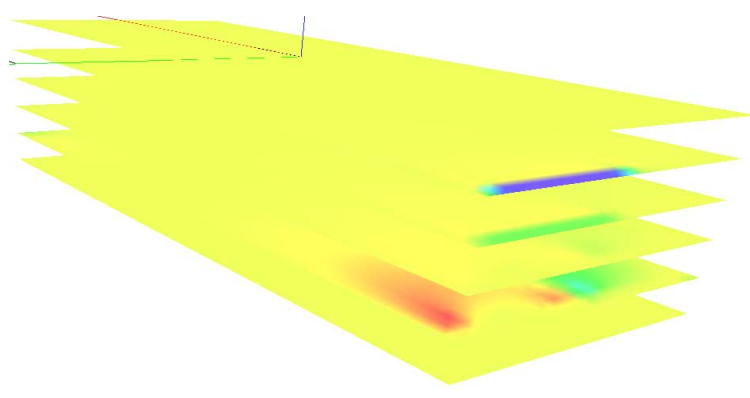

(c)

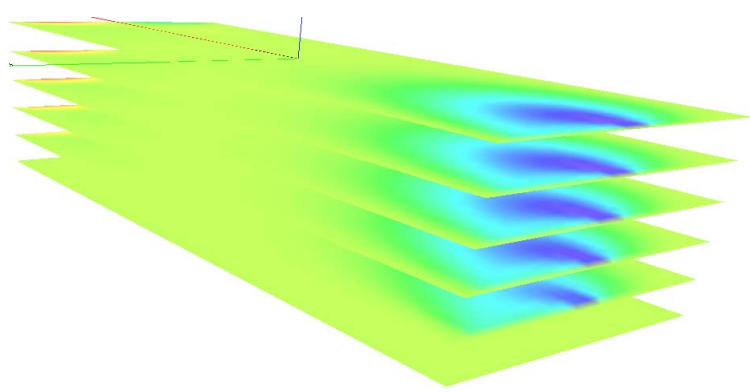

(b)

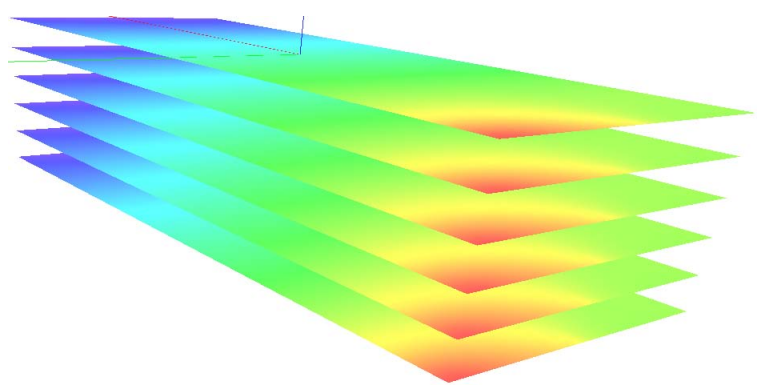

(d)

Figura 6.31: Simulação numérica do escoamento em um degrau: a) campo da componente de velocidade na direção $x, \mathrm{~b}$ ) campo da componente de velocidade na direção $y$, b) campo da componente de velocidade na direção $z$ e d) pressão, com $R e=1$.

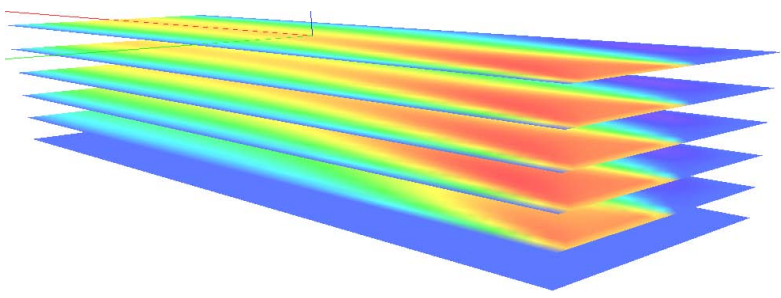

(a)

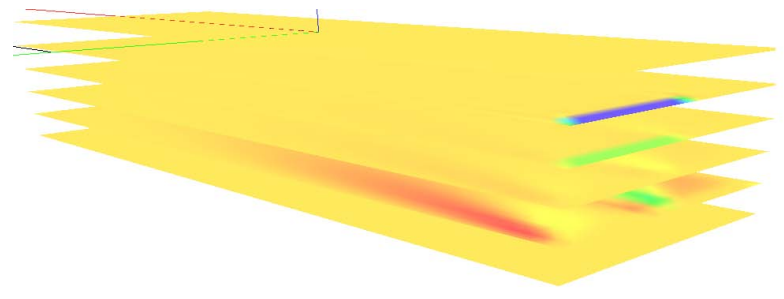

(c)

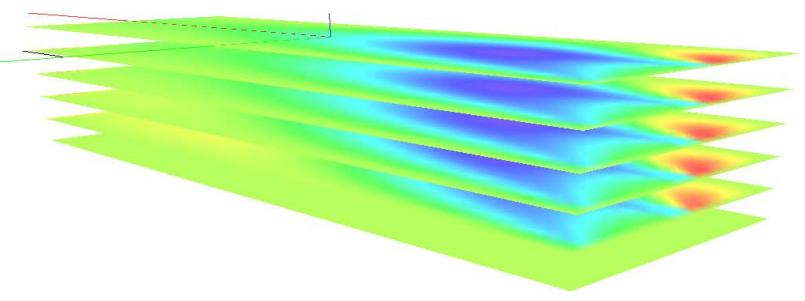

(b)

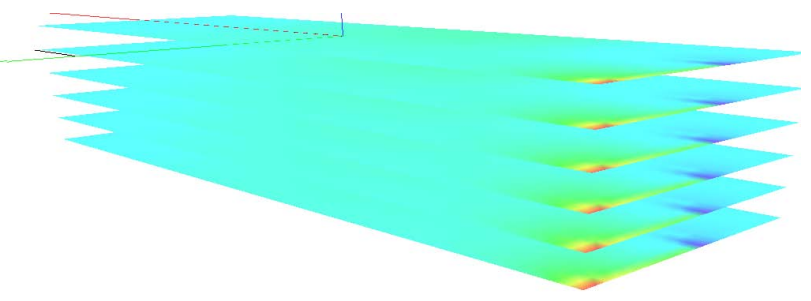

(d)

Figura 6.32: Simulação numérica do escoamento em um degrau: a) campo da componente de velocidade na direção $x$ b) campo da componente de velocidade na direção $y$, c) campo da componente de velocidade na direção $z$ e d) pressão, com $R e=100$. 


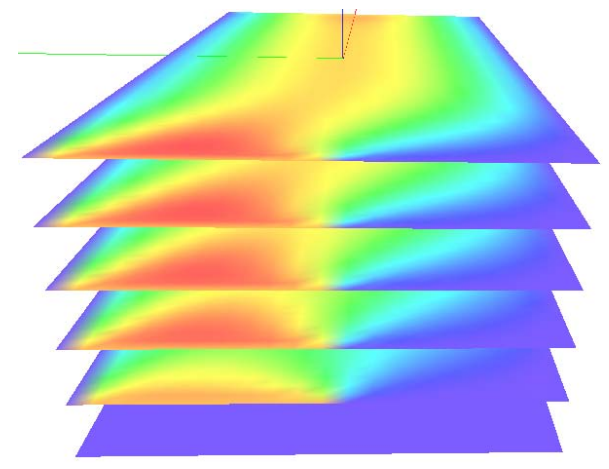

(a)

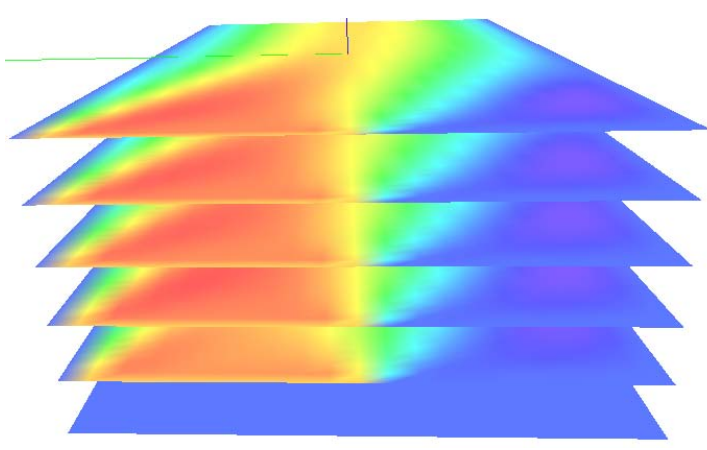

(c)

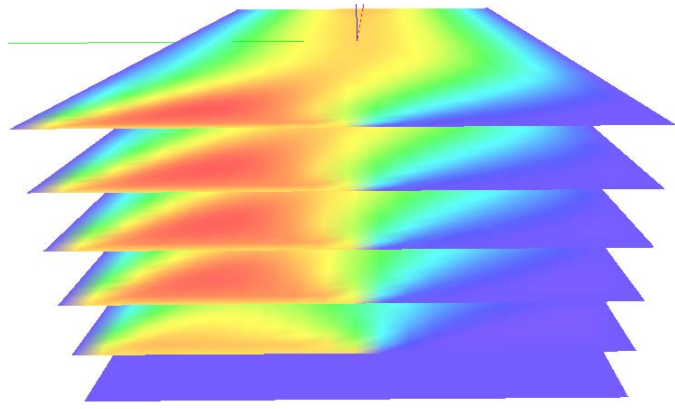

(b)

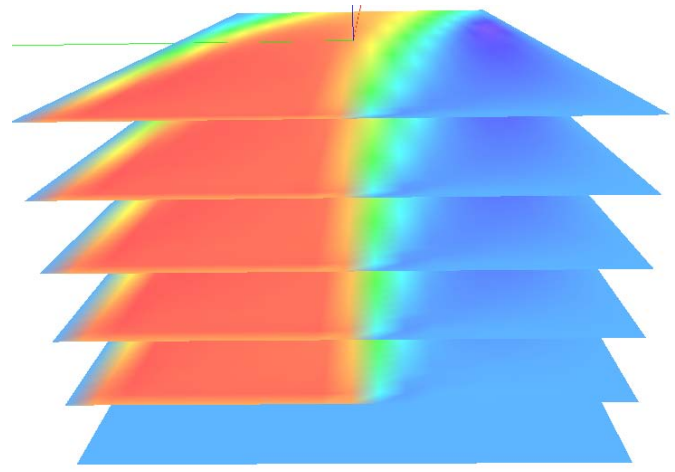

(d)

Figura 6.33: Simulação numérica do escoamento em um degrau com vista na entrada do canal, campo da componente de velocidade na direção $x$ a) $R e=1$, b) $R e=10$, c) $R e=100$ e d) $R e=1000$.

\subsection{Escoamento em um Canal com Inclusão de Esca- das no Domínio}

Nesta seção o caso com inclusão de escadas no domínio será investigado. O objetivo é ter uma simulação de escoamento de fluido em três dimensões chegando mais próximo da realidade para o caso de enchimento de reservatórios. Primeiramente, são definidas as condições iniciais e de contorno utilizadas nas simulações e a representação das escadas no domínio é apresentada. Em seguida, resultados são apresentados analisando-se perfis de velocidade além da pressão obtida na simulação do escoamento. 


\subsubsection{Geometria e Condições de Contorno}

Para a realização das simulações foram impostas condições de não deslizamento para as componentes de velocidade $u, v$ e $w$ nas paredes do domínio e também na região onde contém escadas. A pressão é nula na saída do canal. Na entrada do canal considerou-se $u=1.0 \mathrm{~m} / \mathrm{s}$, para $v$ e $w$ considerou-se velocidades nulas. A componente de velocidade na direção $z$ vale zero na superfície e no fundo do domínio. As condições de contorno utilizadas nestas simulações pode ser vista na Figura 6.34

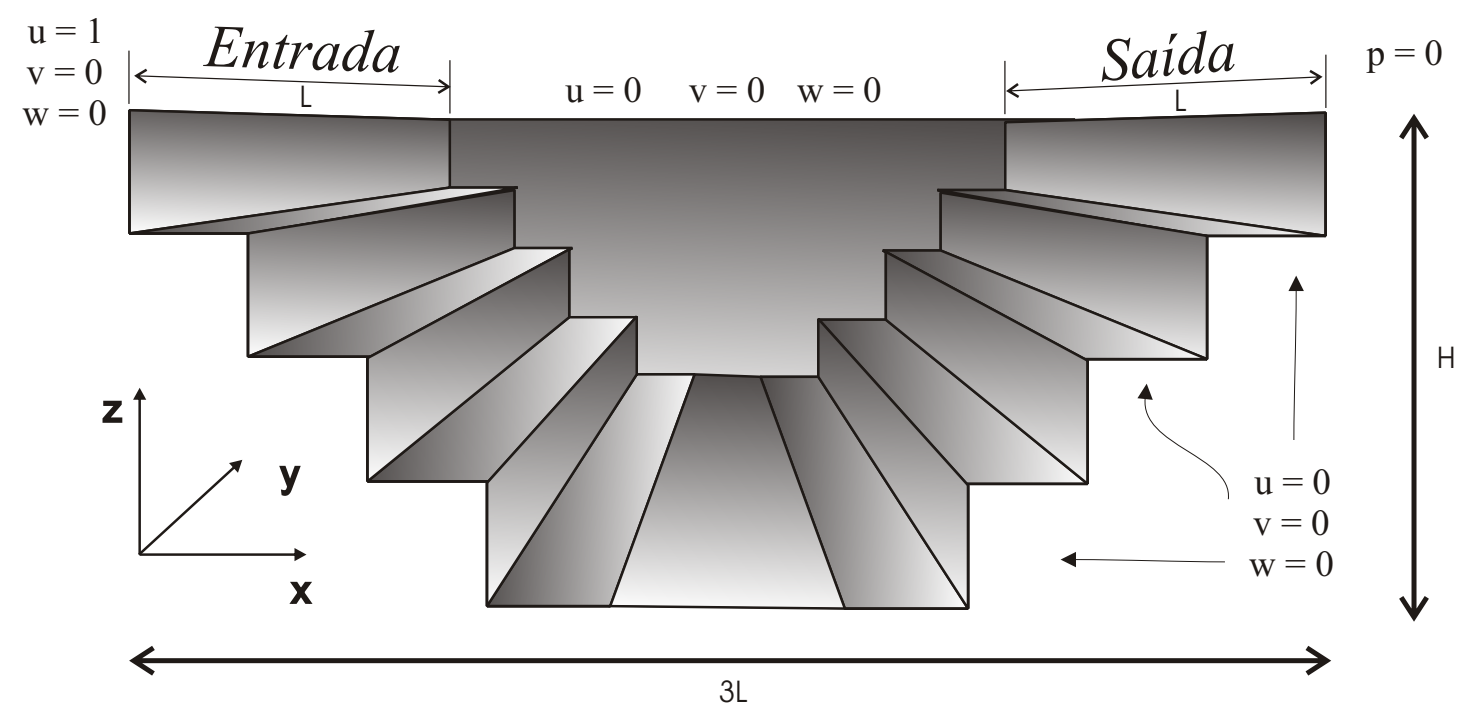

Figura 6.34: Perspectiva na direção y e condições de contorno para o caso de inclusão de escadas no domínio.

Neste caso considerou-se nas simulações a altura do reservatório como sendo $H=1.0$ $m$. Cada degrau da escada na vertical tem altura equivalente a altura de um prisma, comprimento formado por dois prismas como mostrado na Figura 6.35 largura equivalente ao número de prismas existentes na largura do canal.

O modelo para esse escoamento é

- Dimensão do domínio: $3.0 m \times 1.0 m$ x $1.0 \mathrm{~m}$;

- Largura da região de entrada de fluido: $1.0 \mathrm{~m}$;

- Viscosidade : $1.00 \mathrm{Ns} / \mathrm{m}^{2}$;

- Densidade : $1.0 \mathrm{~kg} / \mathrm{m}^{3}$; 
- Parâmetros de escala: $L=1.0 \mathrm{~m}$ e $H=1.0 \mathrm{~m}$;

- Número de Reynolds : 10 e 100.

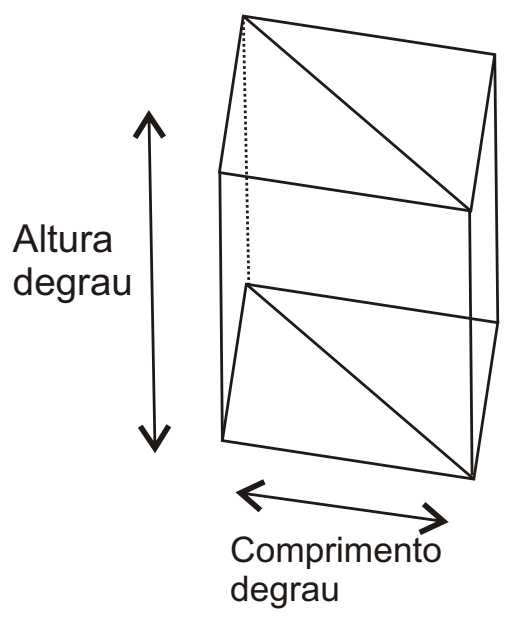

Figura 6.35: Detalhe da dimensão de um degrau na escada.

Os resultados apresentados nas Figuras 6.36 e 6.37 mostram os perfis da componente de velocidade na direção $x$ obtidos na superfície do domínio e na saída de fluido do canal para número de Reynolds 10 e 100. Observa-se que no escoamento, a componente de velocidade na direção $x$ possui altos valores no início do canal, valores intermediários no meio do canal e valores maiores na saída de fluido do canal. Isto se dá pelo fato do domínio apresentar menor profundidade no início e na saída do canal e profundidade maior no meio do canal, como é possível observar pela Figura 6.34, uma perspectiva do domínio.

A Figura 6.38 mostra o perfil de velocidades obtido na superfície do domínio no meio do canal, utilizando número de Reynolds 10 e 100, respectivamente.

A Figura 6.39 mostra o número de iterações e o resíduo obtido pelo método de gradiente conjugado utilizado nas simulações para este caso com inclusão de escadas no domínio, utilizando cinco camadas de prismas.

As simulações caracterizadas pelas Figuras 6.40 e 6.41 mostram o comportamento do escoamento em todos os níveis do domínio para os campos de velocidade e pressão, com número de Reynolds 10 e 100.

A Figura 6.42 mostra a simulação realizada com dez camadas de prismas (onze pontos na direção de $z$ ) da velocidade em todas as direções. 

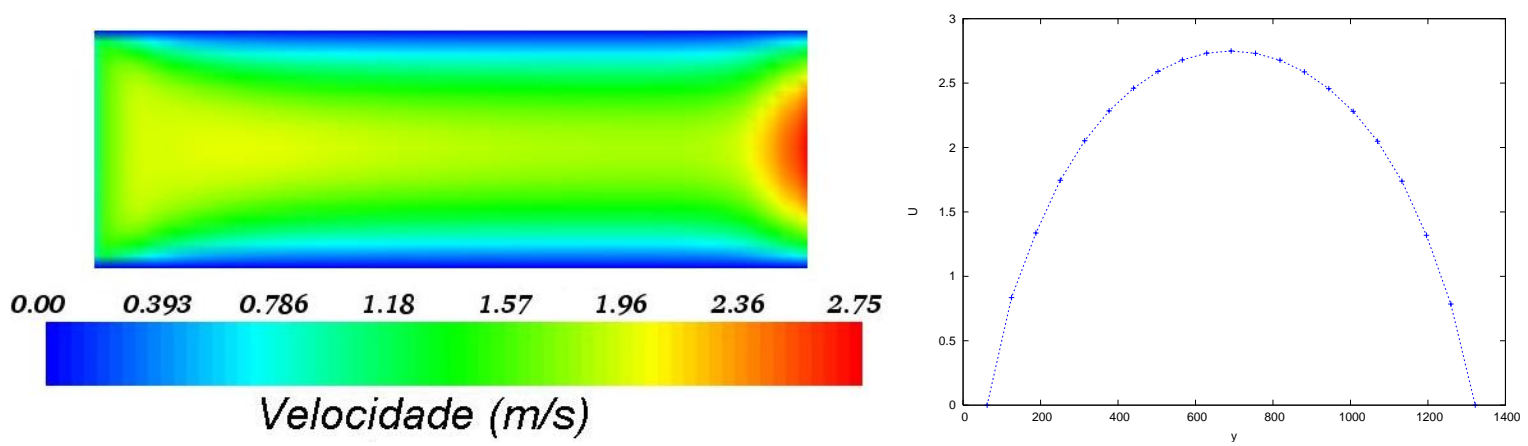

Figura 6.36: Simulação em um domínio com escadas, campo da componente de velocidade na direção $x \operatorname{com} R e=10$, com cinco camadas de prismas.
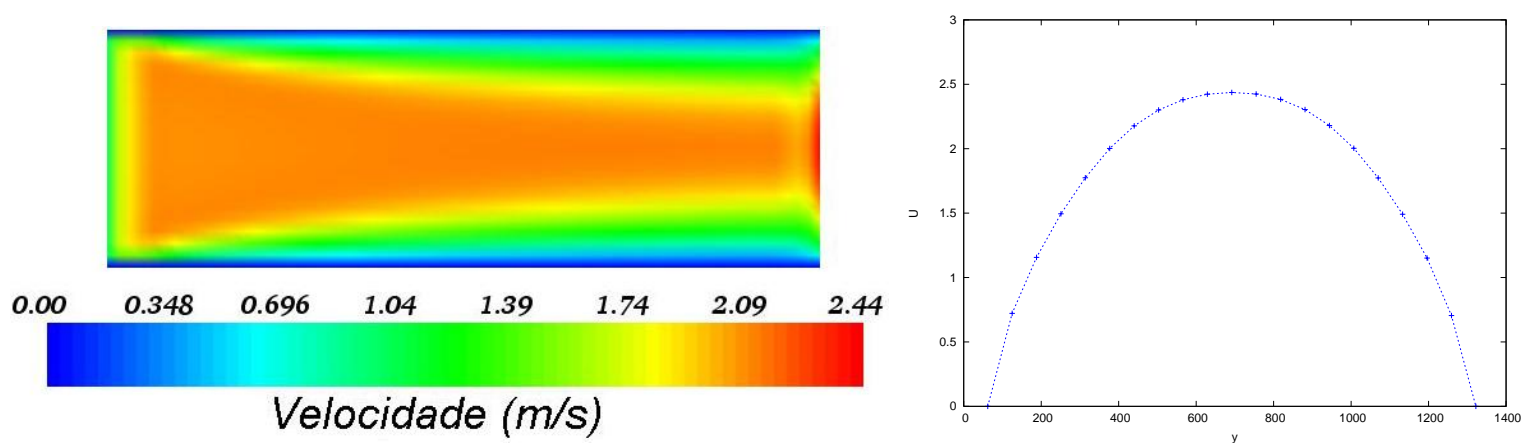

Figura 6.37: Simulação em um domínio com escadas, campo da componente de velocidade na direção $x$ com $R e=100$, com cinco camadas de prismas.

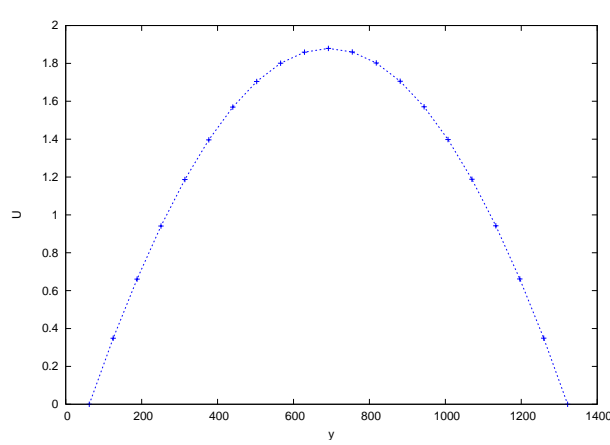

(a)

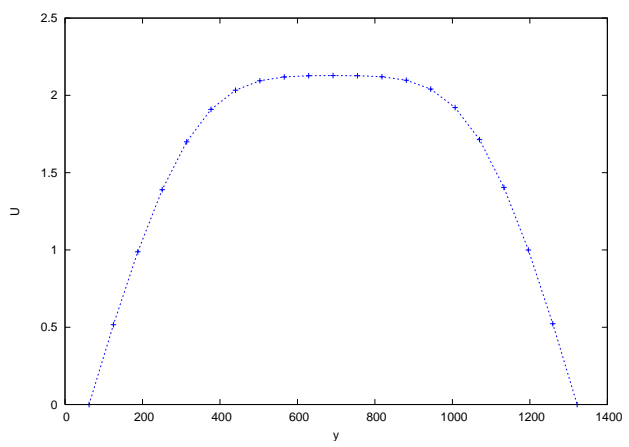

(b)

Figura 6.38: Resultado obtido no meio do canal em um domínio com escadas, campo da componente de velocidade na direção $x$ com cinco camadas de prismas, a) $R e=10$, b) $R e=100$. 


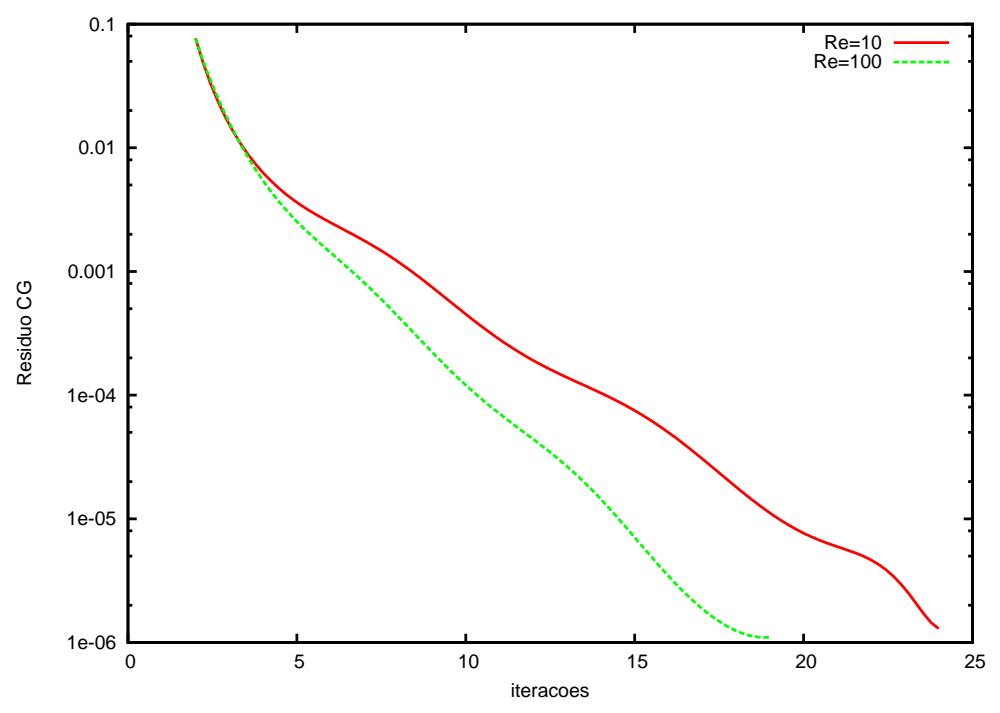

Figura 6.39: Resíduo do método de gradiente conjugado nas iterações.

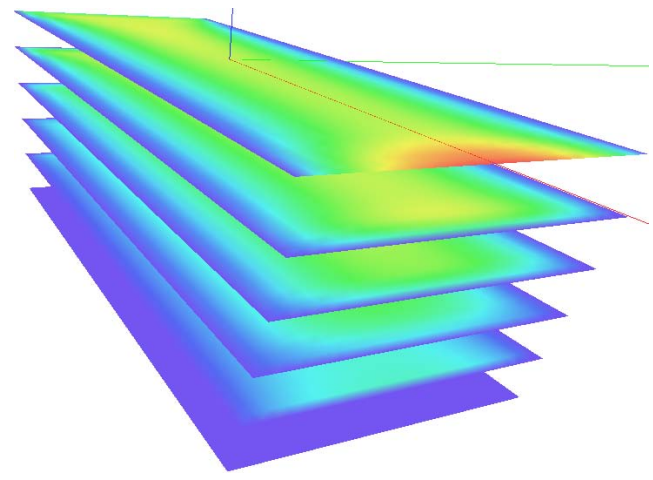

(a)

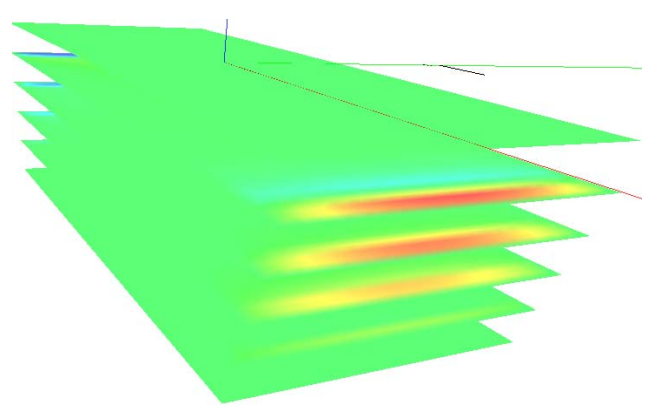

(c)

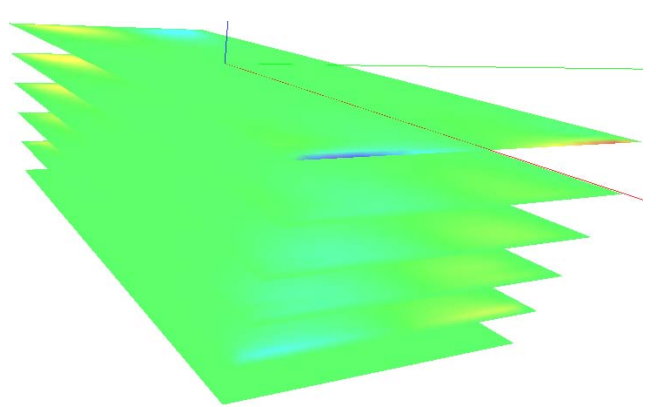

(b)

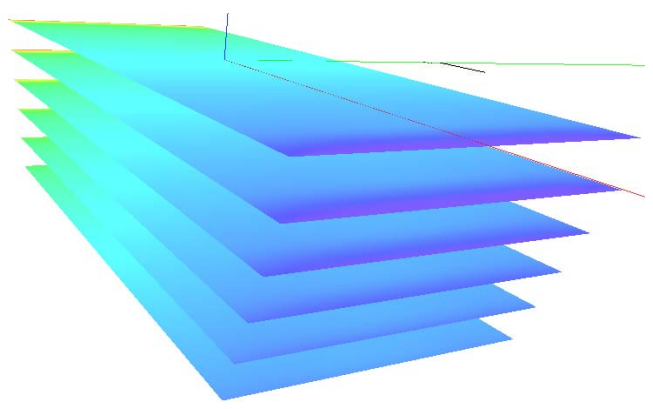

(d)

Figura 6.40: Simulação numérica do escoamento com escadas no domínio: a) campo da componente de velocidade na direção $x, \mathrm{~b}$ ) campo da componente de velocidade na direção $y$, c) campo da componente de velocidade na direção $z$ e d) pressão com $R e=10$. 


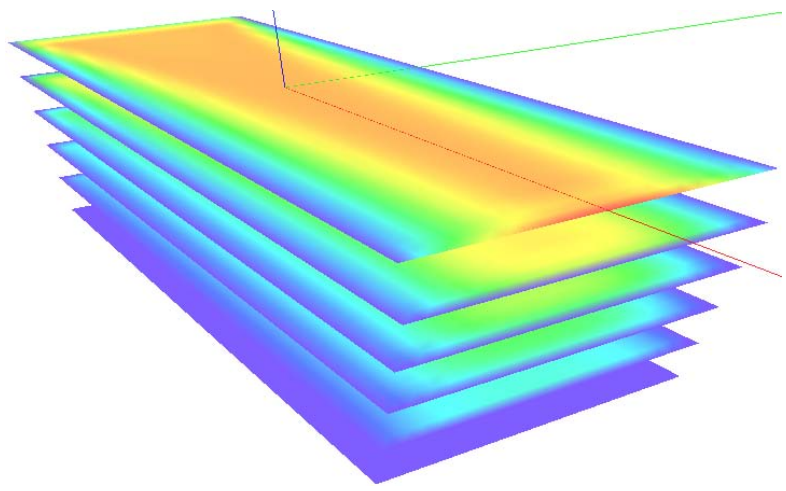

(a)

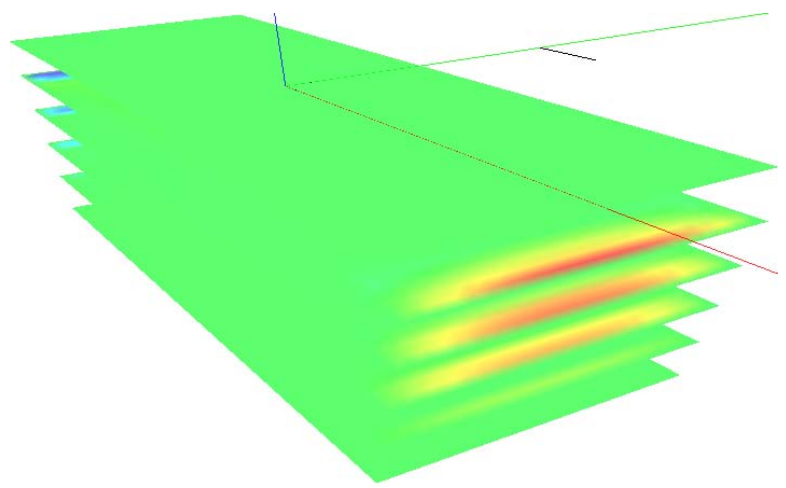

(c)

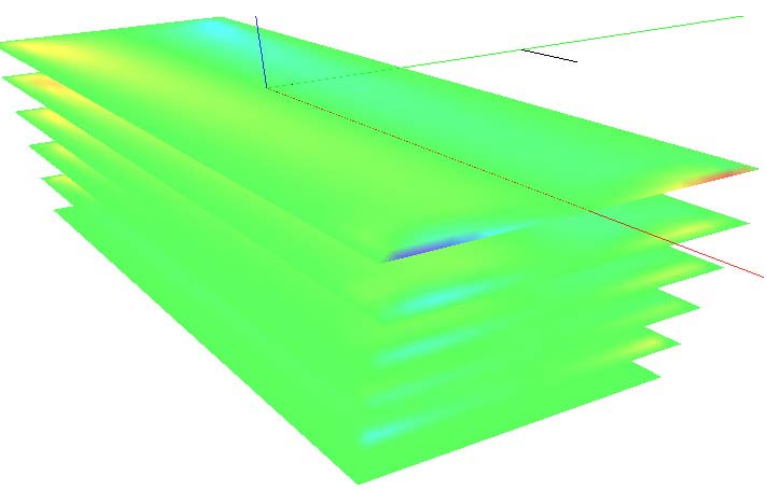

(b)

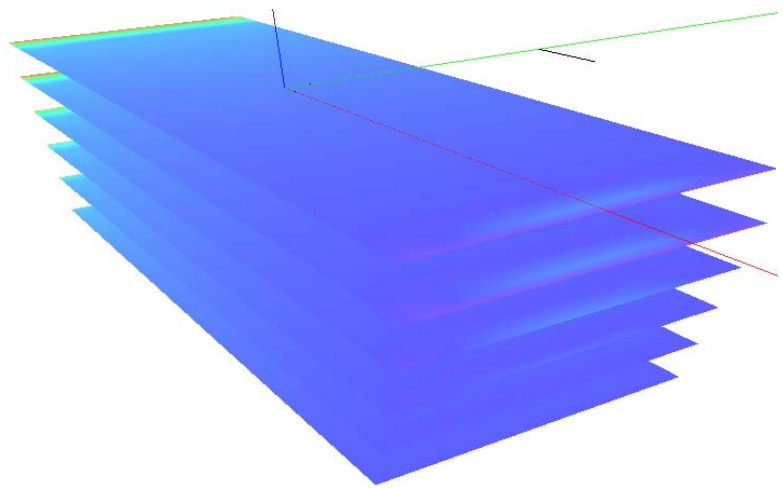

(d)

Figura 6.41: Simulação numérica do escoamento com escadas no domínio: a) campo da componente de velocidade na direção $x, \mathrm{~b}$ ) campo da componente de velocidade na direção $y$, c) campo da componente de velocidade na direção $z$ e d) pressão com $R e=100$. 


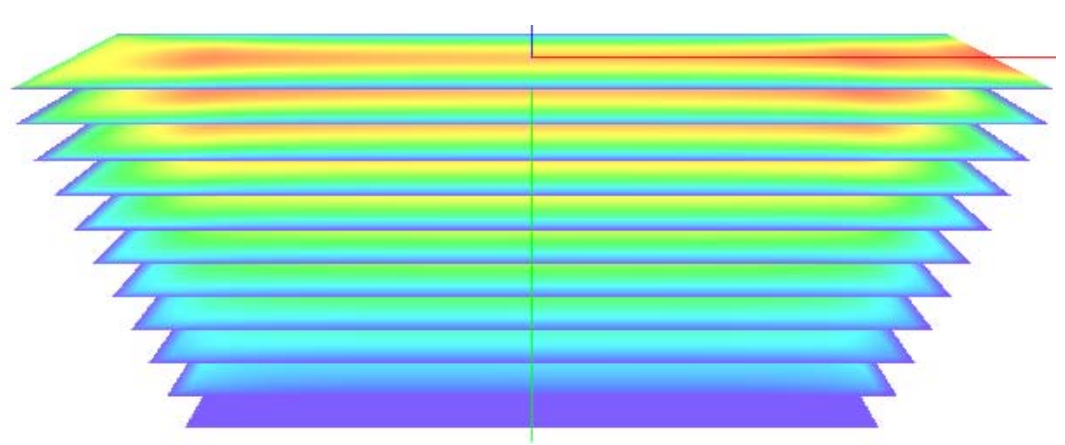

(a)

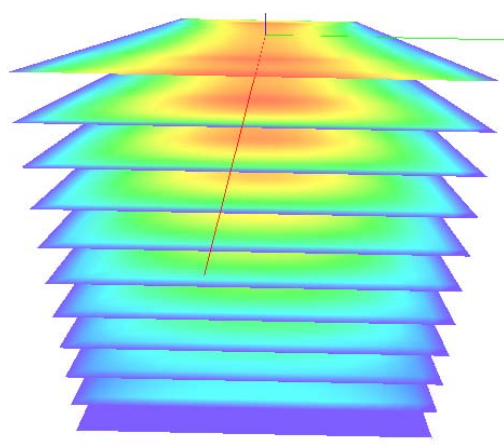

(b)

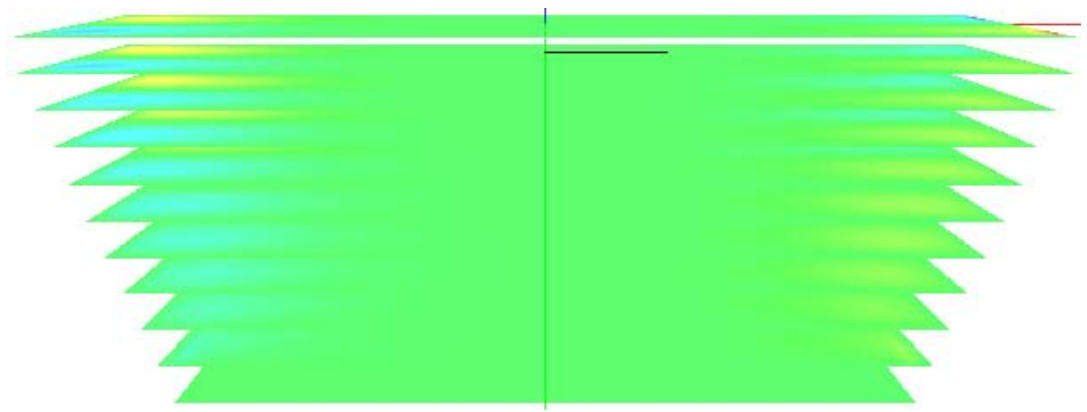

(c)

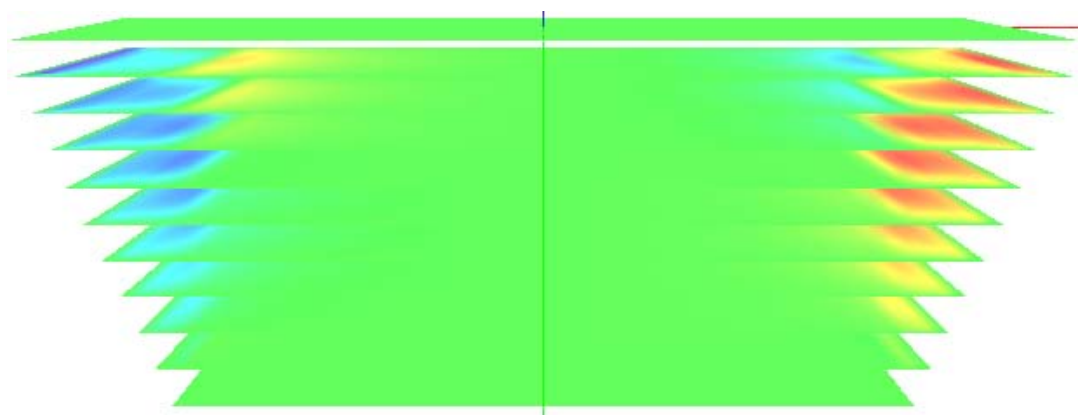

(d)

Figura 6.42: Simulação numérica do escoamento com escadas no domínio: a) campo da componente de velocidade na direção $x, \mathrm{~b}$ ) vista do campo da componente de velocidade na direção $x$ na região de saída de fluido, c) campo da componente de velocidade na direção $y$ e d) campo da componente de velocidade na direção $z$, com $R e=1$. 


\subsection{Considerações Finais}

Neste capítulo foram apresentadas várias simulações de escoamentos de fluido em domínios tridimensionais, utilizando condições de contorno para velocidade e para pressão. Foi apresentado um caso para validação numérica do modelo. A etapa de validação envolveu verificar a presença de um perfil parabólico em relação as velocidades ao longo das camadas do modelo. O modelo desenvolvido mostrou-se convergente quando comparado com a solução analítica.

As equações foram discretizadas espacialmente pelo método de elementos finitos, por um esquema semi-implícito. Para os cálculos das velocidades e pressão foi utilizado o método baseado na decomposição LU. O desacoplamento da pressão possibilitou o uso do simulador 2D já existente para o cálculo desta na superfície do modelo tridimensional. Contudo, obtendo o gradiente de pressão no caso bidimensional, este é atualizado nos níveis inferiores. Esta estratégia para resolução das equações de Navier-Stokes produziu resultados satisfatórios como foi observado na realização das simulações.

Casos diferentes de simulação foram investigados e mostraram-se coerentes com os resultados esperados. Isto mostrou a flexibilidade da ferramenta desenvolvida. 


\section{Capítulo 7 \\ Conclusões}

Neste capítulo são apresentados uma síntese deste trabalho de mestrado, algumas conclusões e propostas para trabalhos futuros.

\subsection{Síntese do Trabalho Desenvolvido}

O objetivo desse trabalho foi desenvolver uma ferramenta para simular escoamento de fluido para aplicação em enchimentos de compartimentos de reservatórios, com um fluido newtoniano incompressível, e usando o método de elementos finitos e malhas estruturadas e não estruturadas. Para implementação da solução numérica foram utilizados o método de elementos finitos e uma estrutura de dados topológica denominada OF - Opposite Face.

Dessa forma, para a implementação da ferramenta, algumas tarefas preliminares foram necessárias, tais como a apresentação das equações governantes do escoamento de fluidos em geral, a determinação de um processo de solução e a especificação das etapas do processo de solução das equações.

No Capítulo 2 foi apresentada uma discussão sobre as principais técnicas numéricas desenvolvidas para aproximar fenômenos físicos e trabalhos encontrados na literatura relevantes a este.

No Capítulo 3 foram apresentados alguns resultados e definições importantes utilizados na derivação das equações governantes do escoamento de fluidos, como o conceito de 
derivada material. Em seguida foram deduzidas, a partir de princípios físicos, as equações de conservação, conhecidas como equação da conservação de massa (ou continuidade) e equação de balanço da quantidade de movimento. As condições de contorno utilizadas na simulação de escoamento de fluidos foram definidas.

No Capítulo 4 o método de elementos finitos utilizado na discretização das equações de Navier-Stokes foi abordado. O método de Galerkin foi apresentado para discretizar as equações de Navier-Stokes no espaço e o método semi-lagrangeano para discretizar os termos convectivos. As matrizes resultantes da discretização para cada elemento foram definidas, assim como as fórmulas de integração numérica. Foi apresentada uma estratégia para solução do sistema de equações resultante da discretização das equações. Pelo fato das equações apresentarem grande número de equações, técnicas computacionais devem ser utilizadas para diminuir custos computacionais como tempo de processamento de dados e uso de memória. Desta forma, a escolha por um método desacoplado baseado em decomposição LU tornou-se a melhor opção.

O Capítulo 5 apresentou a ferramenta desenvolvida para a resolução das equações e detalhes de sua implementação. O cálculo da pressão total no escoamento é feita fazendose uma aproximação hidrostática, ou seja, é feita uma distribuição da pressão da superfície para os níveis inferiores.

Finalmente, de modo a validar o código desenvolvido e a classificar os resultados gerados como confiáveis, uma comparação entre os resultados obtidos com a ferramenta desenvolvida e uma solução analítica foi apresentada no Capítulo 6. Consequentemente, alguns resultados obtidos com a simulação de escoamento de fluido de algumas geometrias foram apresentados e analisados.

\subsection{Conclusões}

O objetivo desse trabalho foi o desenvolvimento de uma ferramenta de simulação de escoamento de fluidos em domínios tridimensionais. Para a implementação do módulo de simulação foram utilizados o método de elementos finitos para a discretização das equações, e uma estrutura de dados topológica.

O ambiente de simulação foi validado considerando-se o problema de escoamento de fluido em um canal, o resultado foi comparado com uma solução analítica, o qual mostrou estar em concordância. Em seguida uma verificação da conservação de massa do sistema foi realizado mostrando consistência. 
O esquema de desacoplamento da pressão utilizando o método de projeção baseado em decomposição LU juntamente com a técnica de aproximação lumped reduziu a complexidade de resolução do problema, resultando em um esquema numérico computacionalmente mais eficiente, pois o gradiente da pressão é calculado de modo independente a cada iteração. Em seguida, a correção dos valores das velocidades é efetuada em conformidade com a equação da continuidade, mantendo o campo de divergência nulo. A solução dos sistemas lineares é obtida utilizando o método dos gradientes conjugados muito utilizado para matrizes de coeficientes simétricas e positivas definidas.

Com a utilização do paradigma de orientação a objetos foi possível implementar um sistema de simulação de fácil manutenção e desenvolvimento futuro, propiciando a incorporação de novas propriedades inerentes ao domínio da aplicação.

Em geral, pode ser concluído que as simulações com o software GesarSim3D, produziram bons resultados conforme o esperado.

\subsection{Trabalhos Futuros}

Algumas sugestões para trabalhos futuros podem ser apresentadas. Dentre elas existe a possibilidade de estender o método proposto, considerando um modelo tridimensional completo, fazendo-se o cálculo da pressão tridimensional.

Uma outra proposta para trabalho futuro consiste em acoplar uma equação de temperatura e também de espécie química como oxigênio dissolvido, demanda bioquímica de oxigênio entre outras propriedades, e desta forma avaliar diversos modelos de qualidade de água.

Uma outra possibilidade para trabalho futuro seria desenvolver um modelo de turbulência para tratamentos de casos de simulação com altos números de Reynolds.

Por fim considerar geometrias mais complexas e sofisticadas para tratamento de casos de simulações do enchimento de compartimentos de reservatórios. 


\section{Referências Bibliográficas}

[Anderson, 1995] Anderson, J. D. (1995). Computational Fluid Dynamics. McGraw-Hill N.Y.

[Aris, 1962] Aris, R. (1962). Vectors, tensors, and the basic equation of fluid mechanics. Dover.

[Auada, 1997] Auada, R. B. (1997). Utilização de malhas não estruturadas em dinâmica dos fluidos computacional. PhD thesis, Escola Politécnica - USP, São Paulo.

[Baker, 1983] Baker, A. J. (1983). Finite element computational fluid mechanics. McGrawHill, New York, 510p.

[Baliga and Pantankar, 1981] Baliga, B. R. and Pantankar, S. V. (1981). A new finite element formulation for convection-diffusion problems. Numerical Heat Transfer, 3:393409.

[Baliga et al., 1983] Baliga, B. R., Phan, T. T., and Pantankar, S. V. (1983). Solution of some two-dimensional incompressible fluid flow anf heat transfer problems using a control-volume finite-element method. Numerical Heat Transfer, 6:263-282.

[Basha, 1997] Basha, H. A. (1997). Analytical model of two-dimensional dispersion in laterally nonuniform axial velocity distributions. Journal of Hydrologic Engineering, 123:853-862.

[Batchelor, 1970] Batchelor, G. K. (1970). An Introduction to Fluid Dynamics. Cambridge.

[Becker et al., 1981] Becker, E. B., Carey, G. F., and Oden, J. T. (1981). Finite Elements An Introduction. Dover.

[Berger and Stockstill, 1995] Berger, R. C. and Stockstill, R. L. (1995). Finite-element 3d model for high-velocity channels. Journal of Environmental Engineering, 121:710-716. 
[Cecchi et al., 1998] Cecchi, M. M., Pica, A., and Secco, E. (1998). A projection method for shallow water equations. International Journal for Numerical Methods in Fluids, $27: 81-95$.

[Chang et al., 2002] Chang, W., Giraldo, F., and Perot, B. (2002). Analysis of an exact fractional step method. Journal of Computational Physics, 180:183-199.

[Chung, 1978] Chung, T. J. (1978). Finite element analysis in fluid dynamics. McGrawHill, New York, 378p.

[Connor and Brebbia, 1976] Connor, J. and Brebbia, C. (1976). Finite Element Techniques for Fluid Flow. London, 330 p. Butterworth and Co (Publishers) Ltda.

[Davies, 1980] Davies, A. J. (1980). The finite element method: a first approach. Claredon Press - Oxford.

[Demuren, 1991] Demuren, A. O. (1991). Development of a mathematical model for sediment transport in meandering rivers. Water Resources Research, Report. n.693, Institute for Hydromechanics, University of Karlsruhe, Karlsruhe, Alemanha.

[Demuren, 1989] Demuren, O. A. (1989). Calculation of sediment transport in meandering channels. In: Tech session A - IAHR Congress, 23, International Association for Hydraulic Research, Delft, Holanda.

[Demuren and Rodi, 1987] Demuren, O. A. and Rodi, W. (1987). Calculation of flow and pollutant dispersion in meandering channels. Journal of Fluid Mechanics, Cambridge, U. K., 172:63-92.

[Ferreira, 2001] Ferreira, V. G. (2001). Análise e implementação de esquemas de convecção e modelos de turbulência para simulação de escoamentos incompressíveis envolvendo superficies livres. PhD thesis, Biblioteca Digital de Teses e Dissertações-www.teses.usp.br, ICMC-USP-São Carlos, Brasil.

[Ferziger and Períc, 1999] Ferziger, J. H. and Períc, M. (1999). Computational Methods for Fluid Dynamics. 2. Heidelberg, Springer-Verlag.

[Flecther, 1992] Flecther, C. A. (1992). Computational Techniques for Fluid Flows. Heidelberg, Springer-Verlag.

[Fortuna, 2000] Fortuna, A. O. (2000). Técnicas Computacionais para Dinâmica dos Fluidos. Edusp.

[Karpik and Crockett, 1997] Karpik, S. R. and Crockett, S. R. (1997). Semi-lagrangian algorithm for two-dimensional advection-diffusion equation on curvilinear coordinate meshes. Journal of Hydraulic Engineering, 123:389-401. 
[Lee et al., 2001] Lee, M. J., Oh, B. D., and Kim, Y. B. (2001). Canonical fractionalstep methods and consistent boundary conditions for the incompressible navier-stokes equations. Journal of Computational Physics, 168:73-100.

[Lin and Falconer, 1996] Lin, B. L. and Falconer, R. A. (1996). Numerical modelling of three-dimensional suspended sediment for estuarine and coastal waters. Journal of $\mathrm{Hy}$ draulic Research, 34, n.4:435-456.

[Lu and Wai, 1998] Lu, Q. and Wai, O. W. H. (1998). An efficient operator splitting for three-dimensional hydrodynamic computations. Journal of Numerical Methods in Fluids, 26:771-789.

[Maliska, 1995] Maliska, C. A. (1995). Transferência de Calor e Mecânica dos Fluidos Computacional. LTC Editora - Rio de Janeiro.

[Mohammad, 2003] Mohammad, A. G. (2003). Books reviews: silting and desilting of reservoirs, by Dan G. Batuca and Jan M. Jordaan. Journal of Hydraulic Engineering, 126 , n. $11: 874-875$.

[Oden et al., 1998] Oden, J. T., Babuska, I., and Baumann, C. E. (1998). A discontinuous hp finite element method for diffusion problems. Journal of Computational Physics, 146:491-519.

[Oden and Wellford, 1972] Oden, J. T. and Wellford, L. C. J. (1972). Analysis of viscous flow by the finite element method. Journal AIAA, 10:1590-1599.

[Odgaard and Bergs, 1988] Odgaard, A. J. and Bergs, M. A. (1988). Flow process in a curved alluvial channel. Water Resources Research, 24, n.1:45-56.

[Panton, 1984] Panton, R. L. (1984). Incompressible flow. John Wiley \& Sons.

[Patankar and Spalding, 1972] Patankar, S. V. and Spalding, D. B. (1972). A calculation procedure for heat, mass and momentum tranfer in three-dimensional parabolic flows. Int. Journal of Heat and Mass Transfer, 15:1787-1806.

[Peyret and Taylor, 1983] Peyret, R. and Taylor, T. D. (1983). Computational Methods for Fluid Flow. Springer-Verlag.

[Prakash and Pantankar, 1986] Prakash, C. and Pantankar, S. V. (1986). A control volume-based finite-element method for solving the Navier-Stokes equation using equalorder velocity-pressure interpolation. Numerical Heat Transfer, 9:253-276.

[Rajar and Cetina, 1997a] Rajar, R. and Cetina, M. (1997a). Hydrodynamic and water quality modelling: an experience. Ecological Modelling, 101:195-207.

[Rajar and Cetina, 1997b] Rajar, R. and Cetina, M. (1997b). Hydrodynamic and water quality modelling: case studies. Ecological Modelling, 101:209-228. 
[Rijn, 1987] Rijn, L. C. V. (1987). Mathematical modelling of morphological process in the case of suspended sediment transport. Delft Hydr:Communication No.382.

[Silva, 1998] Silva, J. B. C. (1998). Simulação numérica de escoamentos de fluidos pelo método de elementos finitos baseado em volumes de controle. PhD thesis, Tese de Doutorado, UNICAMP Faculdade de Engenharia Mecânica, Campinas-SP, Brasil, 159 p.

[Sinha and Odgaard, 1998] Sinha, S. K. and Odgaard, F. S. A. J. (1998). Threedimensional numerical model for flow through natural rivers. Journal of Environmental Engineering, 124:13-24.

[Slobodcicov, 2003] Slobodcicov, I. (2003). Implementação em paralelo do método dos elementos finitos para as equações de águas rasas. Master's thesis, Universidade Federal do Rio de janeiro.

[Soares, 2003] Soares, C. B. P. (2003). Modelagem e Simulação de Sistemas Aquáticos em Ambiente de Geoprocessamento. PhD thesis, Escola de Química -Universidade Federal do Rio de Janeiro - UFRJ, Brasil.

[Sousa, 2005] Sousa, F. S. (2005). Simulação de escoamentos multifásicos em malhas não estruturadas. PhD thesis, Biblioteca Digital de Teses e Dissertações-www.teses.usp.br, ICMC-USP-São Carlos, Brasil.

[Sousa, 2006] Sousa, L. B. S. (2006). Estudo experimental e modelagem numérica do escoamento e do assoreamento em uma armadilha de sedimentos. PhD thesis, Biblioteca Digital de Teses e Dissertações-www.teses.usp.br, ICMC-USP-São Carlos, Brasil.

[Sparrow et al., 1988] Sparrow, E. M., Schneider, G. E., and Pletcher, R. H. (1988). Handbook of Numerical Heat Transfer. John Wiley \& Sons.

[Stockstill et al., 1997] Stockstill, R. L., Berger, R. C., and Nece, R. E. (1997). Twodimensional flow models for trapezoidal high-velocity channels. Journal of Hydraulic Engineering, 123:844-852.

[Vasconcellos, 1999] Vasconcellos, J. F. V. (1999). Um método de volumes finitos usando malhas não estruturadas para o estudo de escoamentos com frentes livres. PhD thesis, Universidade Federal de Santa Catarina, Florianópolis.

[Wang and Adeff, 1986] Wang, S. S. Y. and Adeff, S. E. (1986). Three-dimensional modelling of river sedimentation process. In: International Symposium on river sedimentation, University of Mississippi:Mississippi.

[Whiting, 1999] Whiting, C. H. (1999). Stabilized finite element methods for fluid dynamics using a hierarchical basics. PhD thesis, Faculty of Rensselaer Polytechnic Institute, Troy, New York, USA, 134 pp. 
[Wille, 1998] Wille, S. O. (1998). Nodal operator splitting adaptive finite element algorithms for the navier-stokes equations. International Journal for Numerical Methods in Fluids, 26:959-975.

[Winslow and Harlow, 1967] Winslow, A. M. and Harlow, F. H. (1967). Numerical solution of quasi-linear poisson equation in a non-uniform triangle mesh. Journal of Computational Physics, 2:149-172.

[Wu et al., 2000] Wu, W., Rodi, W., and Wenka, T. (2000). 3d numerical modeling of flow and sediment transport in open channels. Journal of Hydraulic Engineering, 126, n.1:4-15, jan.

[Ye and McCorquodale, 1998] Ye, J. and McCorquodale, J. A. (1998). Simulation of curved open channel flows by 3d hydrodynamic model. Journal of Hydraulic Engineering, 124:687-698.

[Ye and McCorquodate, 1997] Ye, J. and McCorquodate, J. A. (1997). Depth-averaged hydrodynamic model in curvilinear collocated grid. Journal of Hydrodinamic Eng., 123:380-388.

[Zienkiewicz and Taylor, 2000] Zienkiewicz, O. and Taylor, R. (2000). The Finite Element Method Volume 1. Butterworth-Heinemann, 5th edition.

[Zienkiewicz and Cheung, 1965] Zienkiewicz, O. C. and Cheung, Y. K. (1965). Finite elements in the solution of field problems. The Engineer, 507-10 edição. 